
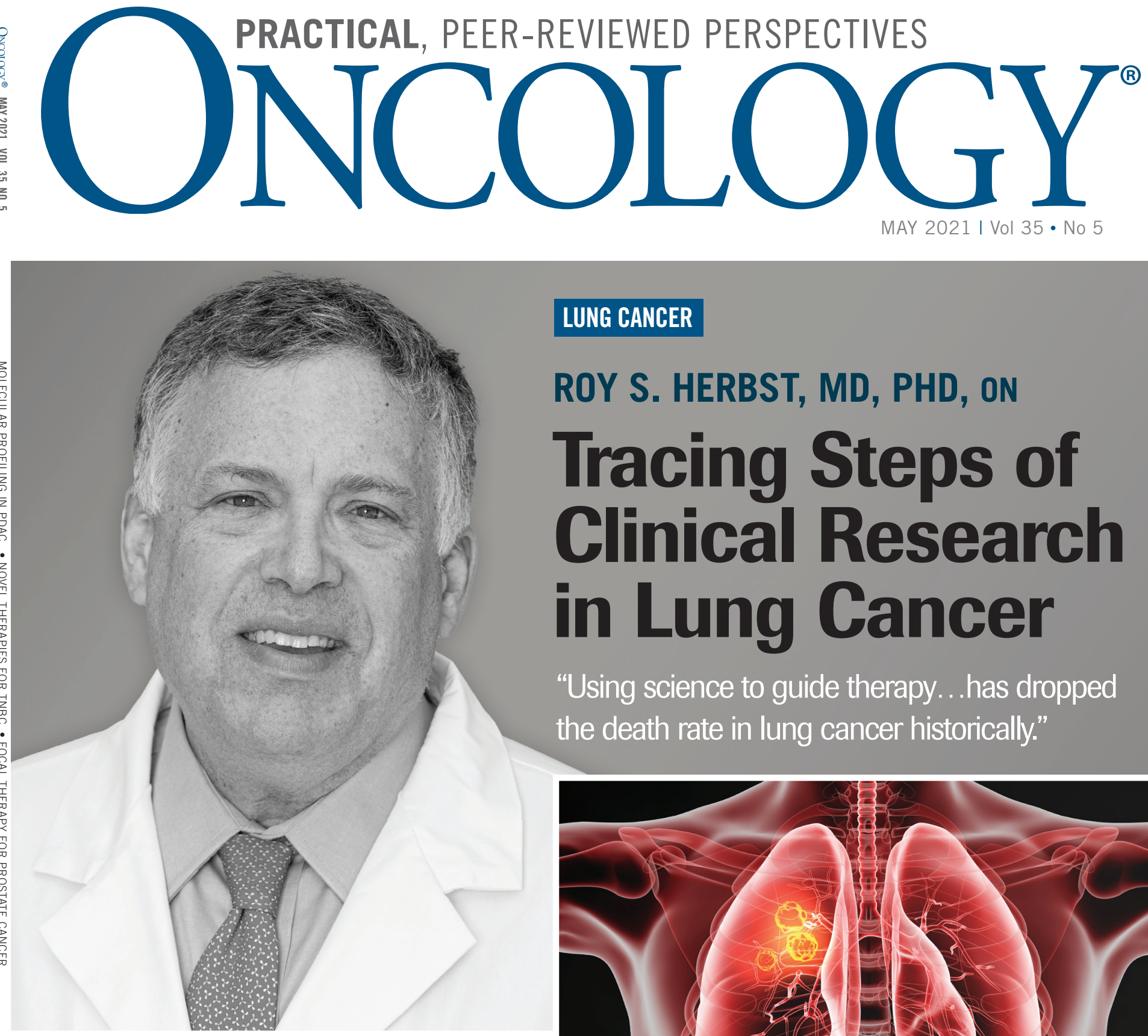

Pancreatic Cancer Molecular Profiling Practices in Pancreatic Adenocarcinoma: Academic vs Community Physicians

Breast Cancer Novel Therapies for Metastatic TripleNegative Breast Cancer: Spotlight on Immunotherapy LUNG CANCER ROY S. HERBST, MD, PHD, ON Tracing Steps of Clinical Research in Lung Cancer "Using science to guide therapy. .. has dropped the death rate in lung cancer historically." and Antibody-Drug Conjugates

Prostate Cancer Primary Focal Therapy for Localized Prostate Cancer: A Review of the Literature

Renal Cell Carcinoma Hand-Foot Skin Reaction Secondary to Sunitinib in a Patient With Metastatic Clear Cell Renal Cell Carcinoma

Hematologic Cancers: CME Transplant-Associated Thrombotic Microangiopathy (TA-TMA): Integrating New Data to Improve Patient Outcomes

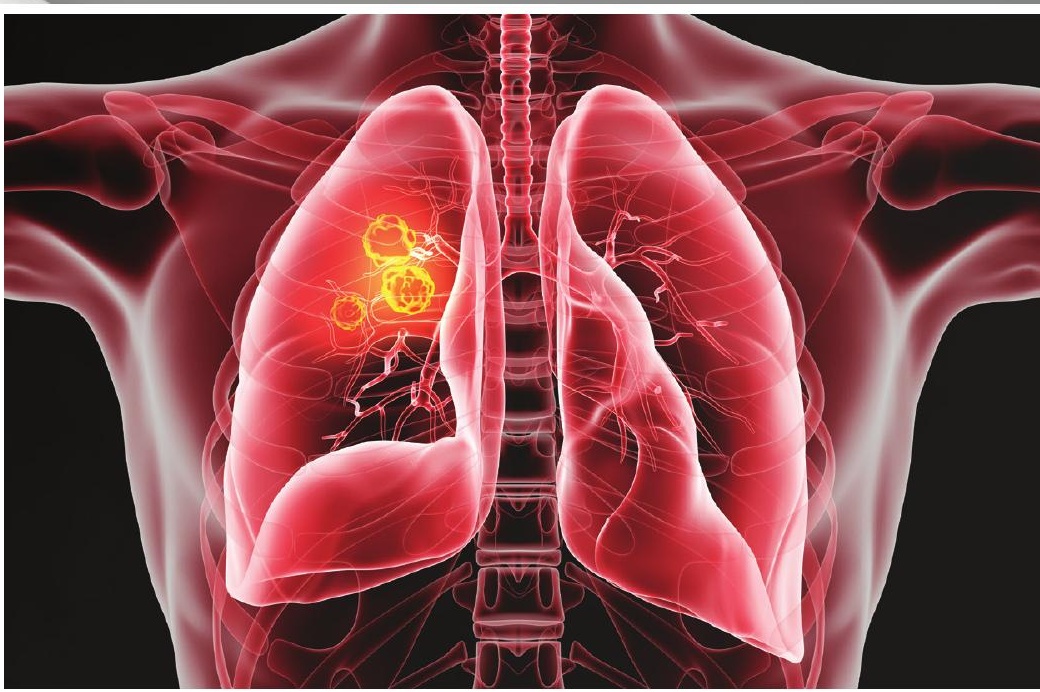




\section{cancervetwork}

Hear timely \& informative insights from some of the leading voices in the field.
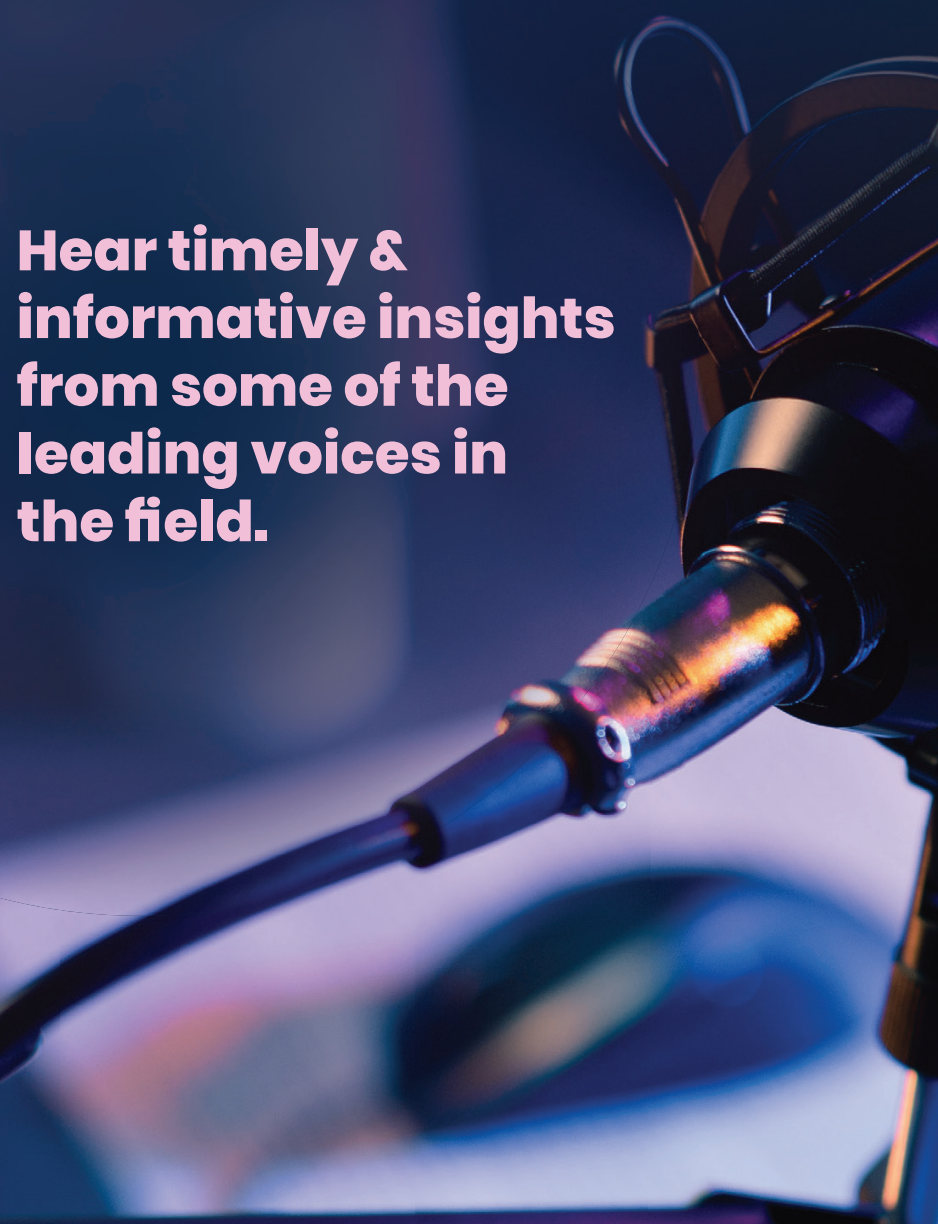

$\because$

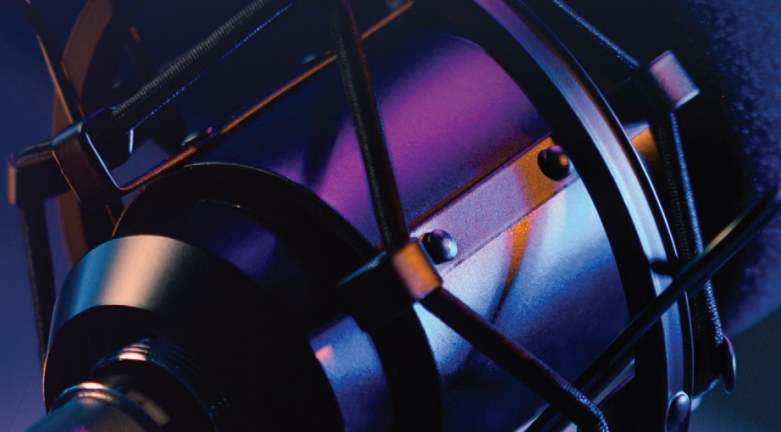

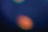

Listen: www.cancernetwork.com/resources/podcasts

Sean to listen

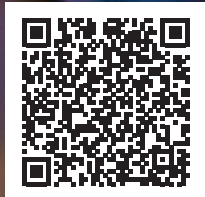


IN THIS ISSUE

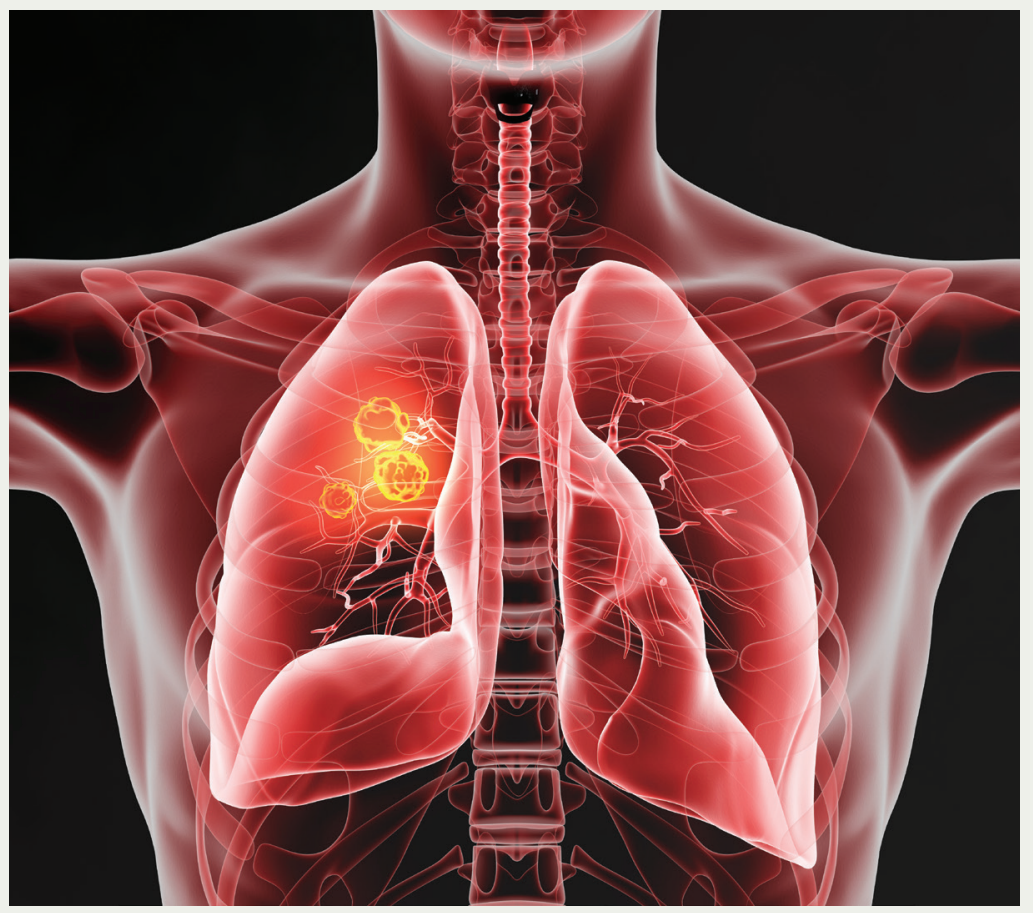

242 Lung Cancer: COVER

\section{$\angle 42$ Tracing Steps of Clinical Research} in Lung Cancer

ONCOLOG $Y^{\circledR}$ sat down with Roy S. Herbst, MD, PhD, who is Ensign Professor of Medicine (medical oncology) and professor of pharmacology, chief of medical oncology, and associate cancer center director for translational science at Yale Cancer Center and Smilow Cancer Hospital, in New Haven, Connecticut, to discuss the research path that led to modern therapy strategies in lung cancer.

238 Chairman's Letter

Exploring Novel Mechanisms of Action-Then and Now

Mike Hennessy Sr

240

Letter to Readers

Next-Generation Sequencing for Advanced Pancreatic Cancer: A Technology Whose Time Has NOT Come

Howard S. Hochster, MD

\section{cancernetwork.com}

Visit CancerNetwork.com, home of the journal ONCOLOGY $Y^{\circledR}$ and a web destination for oncologists seeking expert peer perspectives, podcasts, and other clinically practical features.

\section{VIDEO}

Vivek Subbiah, MD, on

Data From AACR on

Resistance Mechanisms

to KRAS Inhibitors

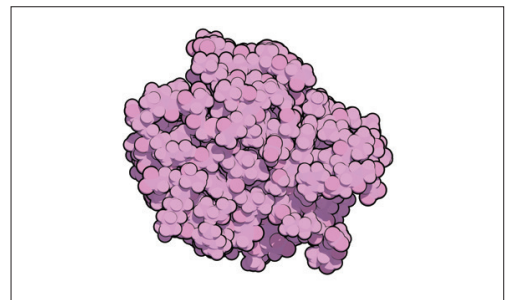

Cancernetwork.com/Subbiah_AACR2021

\section{NEWS}

Phase 3 Trial of Tumor-Treating Fields for NSCLC Impresses at Interim Analysis

Cancernetwork.com/Lunar TTF

\section{FDA APPROVAL}

FDA Approves Loncastuximab Tesirine in Patients With R/R Large B-Cell Lymphoma

Cancernetwork.com/Lonca_approval

Check out our eNewsletter for the latest in oncology. Scan to subscribe.

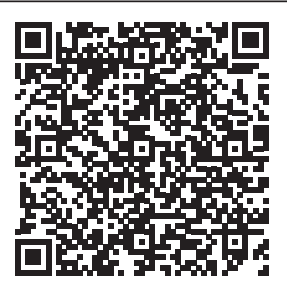


IN THIS ISSUE

Pancreatic Cancer:

Original Research

244 Molecular Profiling

Practices in Pancreatic

Adenocarcinoma:

Academic vs Community Physicians

Christine Y. Chung, DO; Rachael Galvin, DO, MPH; Ella Achenbach;

Oliwier Dziadkowiec, PhD; and

Shiraj Sen, MD, PhD

Breast Cancer: Review 249 Novel Therapies for Metastatic TripleNegative Breast Cancer: Spotlight on Immunotherapy and Antibody-Drug Conjugates

Aiko Nagayama, MD, PhD;

Neelima Vidula, MD; and

Aditya Bardia, MD, MPH

Prostate Cancer: Review 261 Primary Focal Therapy for Localized Prostate Cancer: A Review of the Literature

Mitch Hayes, MD; Michael

Lin-Brande, MD; and Sudhir Isharwal, MD

PERSPECTIVE: Prostate Cancer Focal

Therapy Has Made Great Strides and the Future Remains Bright

Thomas J. Polascik, MD

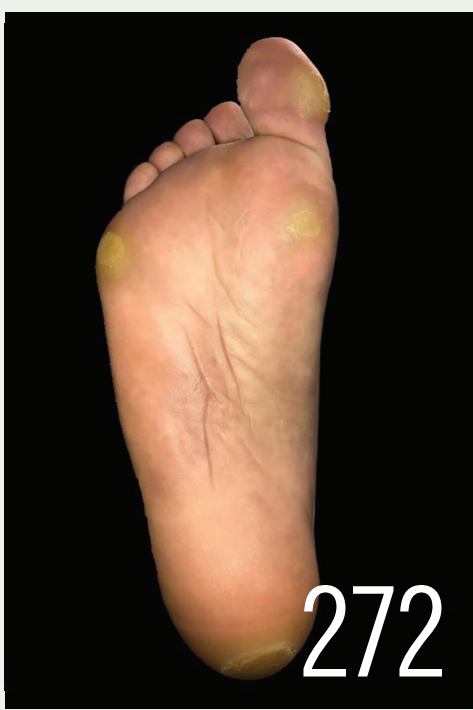

Renal Cell Carcinoma:

Clinical Quandaries

272 Hand-Foot Skin

Reaction Secondary to

Sunitinib in a Patient

With Metastatic

Clear Cell Renal Cell Carcinoma

Karen Férez-Blando, MD;

Francisco J. Castro-Alonso, MD; Judith Dominguez-Cherit, MD;

Maria T. Bourlon, MD, MSc

Careers: Medical

Economics $^{\circledR}$

276 Advanced

Technology Will Help

Drive Reimbursement Change

Stephan Ondra, MD
Multiple Myeloma:

Product Profile

277 Belantamab

Mafodotin

INTERVIEW WITH: Kathryn Maples,

PharmD, BCOP

Prostate Cancer: Around the Practice

280 The Management of Biochemical Recurrence in Prostate Cancer

Multiple Myeloma:

Clinical Consult

284 Targeted Therapy

Treatment Options for

Patients With Relapsed/

Refractory Multiple

Myeloma

Hematologic Cancers:

Continuing Medical

Education

288 Transplant-

Associated Thrombotic

Microangiopathy

(TA-TMA): Integrating

New Data to Improve

Patient Outcomes

Sergio A. Giralt, MD, FACP

Published in affiliation with

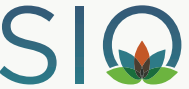

Integrative Oncology

IPER THE EDITORS ARE PLEASED to ANNOUNCE the availability of our new parent company's continuing education activities. gotoper.com We've picked this one especially for our $O N C O L O G Y^{\circledR}$ readers. Go to: https://bit.ly/2IRAknZ

advertisements, advertiser and advertising agency agree to fully defend, indemnity and hold harm ess the publisher, and to pay any judgment, expenses and logalfees incurred by the publisher as a result of said legal action or claim. The publisher reserves the right to reject any advertising which he feels is not in keeping with the publication's standards.

Publisher is not liable for delays in delivery and/or non-deliveryin the event of an act of God, action by any government or quasi-governmental entity, fire, flood, insurrection, riot, explosion, embargo, strikes (whether legal or illegal), labor or material shortage, transportation interruption of any kind, work slow-down, or any conditio beyond the control of publisher affecting production or delivery in any manner.
ONCOLOGY (ISSN 0890-9091) is published monthly by MultiMedia Healthcare LLC, 2 Clarke Drive, Suite 100 Cranbury N08512 Annua subscription rates: US \$237 and Canada \$261; students and nurses, \$96; intepostage paid at Trenton, $\mathrm{N}$ and at additional mailing to Oncology PO Box 457, Cranbury NJ 08512-0457, USA. Publications Mail Agreement № 40612608. Retur Undeliverable Canadian Addresses to: IMEX Global Solutions, PO Box 25542 London ON N6C 6B2. Canadian G.S.T number: R-124213133RT001. Printed in U.S.A.

For address changes, please notify the Circulation Department by visiting www.surveymonkey.com/s/subscriptions, or by mail to ONCOLOGY ( @ $2021 \mathrm{MJH}$ Life Sciences, PO Box 457, Cranbury NN 08512-0457. Send old address, new address and attach a copy of mail label, if possible. 


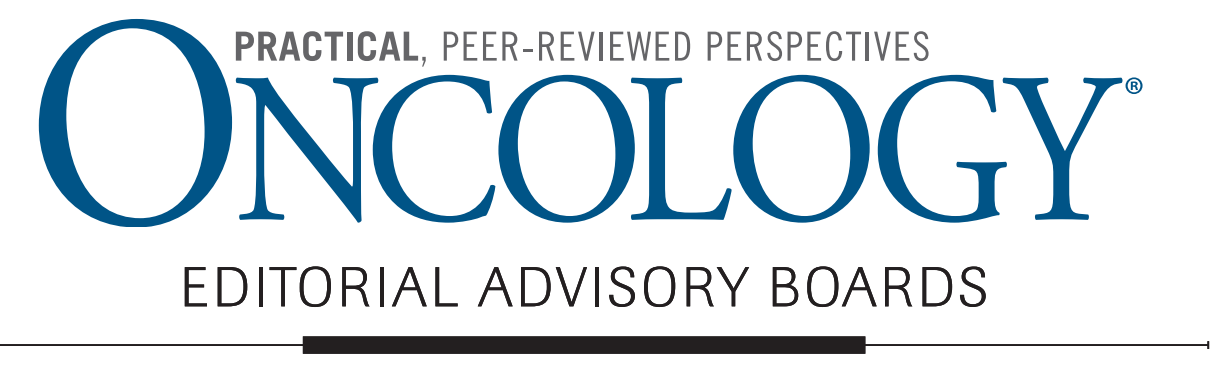

MISSION

STATEMENT

$O N C O L O G Y^{\circledR}$ and its website, CancerNetwork.com, provide oncologists with the practical, timely, clinical information they need to deliver the highest level of care to their patients. Expert authors and peer review ensure the quality of ONCOLOGY ${ }^{\circledR}$ and CancerNetwork.com's articles and features. Focused discussions capture key clinical take-aways for application in today's time-constrained practice environment.

\section{EDITORS-IN-CHIEF}

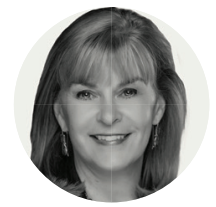

Julie M. Vose, MD, MBA Omaha, NE

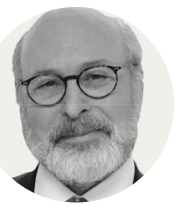

Howard S. Hochster, MD New Brunswick, NJ

\section{EDITORIAL BOARD}

\section{TUMOR CHAIRS}

\section{BREAST CANCER}

Sara A. Hurvitz, MD, Los Angeles, CA

GENITOURINARY CANCER

Robert A. Figlin, MD, Los Angeles, CA
GASTROINTESTINAL CANCER

Tanios S. Bekaii-Saab, MD, Phoenix, AZ

HEAD AND NECK CANCER

Eric J. Sherman, MD, New York, NY
HEMATOLOGIC MALIGNANCIES

C. Ola Landgren, MD, PhD, New York, NY

LUNG CANCER

Joshua M. Bauml, MD, Philadelphia, PA

\section{BOARD MEMBERS}

BREAST CANCER

William J. Gradishar, MD, FACP, Chicago, It Tari King, MD, Boston, MA

Vered Stearns, MD, Baltimore, MD

Melinda L. Telli, MD, Palo Alto, CA

CANCER SURVIVORSHIP

Matthew J. Matasar, MD, MS, New York, NY

COLORECTAL/GASTROINTESTINAL CANCER

Edward Chu, MD, Pittsburgh, PA

Mehmet Sitki Copur, MD, FACP, Omaha, NE

Daniel Haller, MD, Philadelphia, PA

John L. Marshall, MD, Washington, DC

Shubham Pant, MD, Houston, TX

Matthew B. Yurgelun, MD, Boston, MA

Mehmet Sitki Copur, MD, FACP, Omaha, NE

GENITOURINARY CANCER

L. Michael Glodé, MD, FACP, Denver, CO

Paul Mathew, MD, Boston, MA

Elisabeth Heath, MD, FACP, Detroit, MI

William Oh, MD, New York, NY

GYNECOLOGIC ONCOLOGY

Mario M. Leitao Jr, MD, New York, NY

Franco Muggia, MD, New York, NY
HEAD AND NECK CANCER

Apar K. Ganti, MD, MS, FACP, Omaha, NE

HEALTH ECONOMICS

Nora Janjan, MD, Dallas, TX

HEMATOLOGIC MALIGNANCIES

Danielle M. Brander, MD, Durham, NC

Christopher R. Flowers, MD, Houston, TX

Steven T. Rosen, MD, Duarte, CA

Naval G. Daver, MD, Houston, TX

Ehab L. Atallah, MD, Milwaukee, WI

INFECTIOUS DISEASE

Genovefa Papanicolaou, MD, New York, NY

INTEGRATIVE ONCOLOGY

Ting Bao, MD, New York, NY

Linda Carlson, PhD, RPsych, Calgary, Alberta,

Canada

LUNG CANCER

David S. Ettinger, MD, Baltimore, MD

James L. Mulshine, MD, Chicago, IL

Edward S. Kim, MD, Charlotte, NC

Jennifer W. Carlisle, MD, Atlanta, GA

MELANOMA

Richard D. Carvajal, MD, New York, NY

Jason Luke, MD, FACP, Pittsburgh, PA

\author{
NEURO-ONCOLOGY \\ David A. Reardon, MD, Boston, MA \\ Stuart A. Grossman, MD, Baltimore, MD \\ Nicole A. Shonka, MD, Omaha, NE \\ PEDIATRIC ONCOLOGY \\ David G. Poplack, MD, Houston, TX \\ Richard A. Drachtman, MD, New Brunswick, NJ \\ PROSTATE CANCER \\ Tomasz M. Beer, MD, Portland, OR \\ E. David Crawford, MD, Denver, CO \\ Judd W. Moul, MD, FACS, Durham, NC \\ PSYCHO-ONCOLOGY \\ Daniel C. McFarland, DO, New York, NY \\ Michelle Riba, MD, Ann Arbor, MI \\ RADIATION ONCOLOGY \\ Louis Potters, MD, FACR, Hempstead, NY \\ James B. Yu, MD, MHS, New Haven, CT \\ SARCOMA \\ Kenneth Cardona, MD, FACS, Atlanta, GA \\ SUPPORTIVE AND PALLIATIVE CARE \\ Thomas J. Smith, MD, FACP, Baltimore, MD \\ N. Simon Tchekmedyian, MD, Long Beach, CA \\ SURGICAL ONCOLOGY \\ Burton L. Eisenberg, MD, Newport Beach, CA
}

INTERESTED IN SUBMITTING TO ONCOLOGY ${ }^{\circledR}$ ?

Please contact Managing Editor Audrey Sternberg at ASternberg@mjhlifesciences.com for submission guidelines. 


\section{Exploring Novel Mechanisms of Action-Then and Now}

The success of systemic therapies in the treatment of hematologic and solid tumors has revolutionized oncology treatment, with multiple immunotherapy and targeted agents supplanting prior standard-of-care disease management practices. Advances in lung cancer are unrivaled by research in many diseases, making reflection on the last generation of breakthroughs critical in understanding how far the field has come.

"[T]he fact that we could take scientific discoveries...and [translate that to] targeted therapy and immunotherapy is fantastic," Roy S. Herbst, MD, PhD, of Yale Cancer Center and Smilow Cancer Hospital, in New Haven, Connecticut, and cochair of the 22nd Annual International Lung Cancer Congress ${ }^{\circledR}$, hosted by Physicians' Education Resource ${ }^{\circledR}$, LLC 0, said in an interview with ONCOLOGY ${ }^{\circledR}$. "It speaks to how we are bridging the gap and helping people live longer with this disease. That's what gets me up every morning: seeing the science translated to the patient."

In the interview, Herbst takes a deep dive into his career as a lung cancer physician and the research he has conducted. He details the timelines of significant clinical research studies, such as pivotal trials that led to approvals of targeted agents against EGFR and have revolutionized approaches to treating advanced non-small cell lung cancer.

Also in this issue, the ONCOLOGY ${ }^{\circledR}$ editors debuted the first installment of Product Profile, aimed at discovering real-world implications of systemic therapies with new indications. This month, we spoke with Kathryn Maples, PharmD, BCOP, clinical pharmacy specialist of multiple myeloma at Winship Cancer Institute of Emory Healthcare in Atlanta, Georgia, about belantamab mafodotin (Blenrep). In a disease state that saw multiple FDA approvals and designations throughout the preceding year, belantamab mafodotin stood out as being the first B-cell maturation antigen-targeted agent for use in patients with myeloma, offering a new therapeutic mechanism of action in a population of patients who are often treated with multiple lines of therapy throughout the course of their disease.

Aiko Nagayama, MD, PhD, of Keio University School of Medicine in Tokyo, Japan, and her colleagues presented a comprehensive review of novel therapies for triple-negative breast cancer, which has traditionally been associated with poor outcomes as compared with other tumor subtypes. At the forefront, the authors discuss antibody-drug conjugates and immunotherapy agents that have recently been approved.

As always, the articles within these pages and more on breakthroughs in oncology across multidisciplinary specialties can be found at CancerNetwork.com, home of the journal ONCOLOGY ${ }^{\circledR}$.

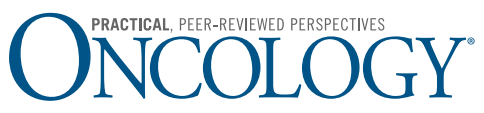

MAY 2021 - VOL. 35 - NO. 5

\section{EDITORIAL}

KRISTIE L. KAHL Vice President, Content AUDREY STERNBERG Managing Editor MATTHEW FOWLER Assistant Editor JENNIFER POTASH Copy Chief RACHELLE LALIBERTE, PAUL SILVERMAN Copy Supervisors KELLY KING Senior Copy Editor

CHENEY BALTZ, GEORGINA CARSON, REBEKAH HARRISON, KIRSTY MACKAY, RON PANAROTTI

Copy Editors

\section{DESIGN \& PRODUCTION}

ROBERT MCGARR Creative Director

KRISTEN MORABITO Art Director

RACHEL KEATLEY Senior Graphic Designer

JONATHAN SEVERN Circulation Director

KEYONNA GRAHAM Production Director

\section{Publishing \& SALES}

BRIAN HAUG Executive Vice President 609-325-4780•bhaug@mmhgroup.com

MICHELLE JANIN Director of Sales

732-429-4316•mjanin@mmhgroup.com

PATRIC PASTORE National Accounts Associate 609-955-1694 • ppastore@mjhlifesciences.com

KRISTEN KOEDERITZ National Accounts Associate KKoederitz@mjhlifesciences.com

\section{AUDIENCE DEVELOPMENT \\ KELLY KEMPER Audience Development Manager}

\section{CORPORATE}

MIKE HENNESSY SR Chairman \& Founder

JACK LEPPING Vice Chairman

MIKE HENNESSY JR President \& CEO

NEIL GLASSER, CPA/CFE Chief Financial Officer

MICHAEL BAER Chief Marketing Officer

JOE PETROZIELLO Executive Vice President, Global Medical Affairs and Corporate Development

SILAS INMAN Senior Vice President, Content

MICHAEL BALL Senior Vice President Operations

JOHN MORICONE Senior Vice President, I.T. \& Enterprise Systems

SHARI LUNDENBERG Vice President, Human Resources \& Administration

CHRIS HENNESSY Vice President, Mergers \& Acquisitions JEFF BROWN Executive Creative Director, Creative Services

\section{SUBSCRIPTIONS}

888-527-7008

AN life sciences“ BRAND 


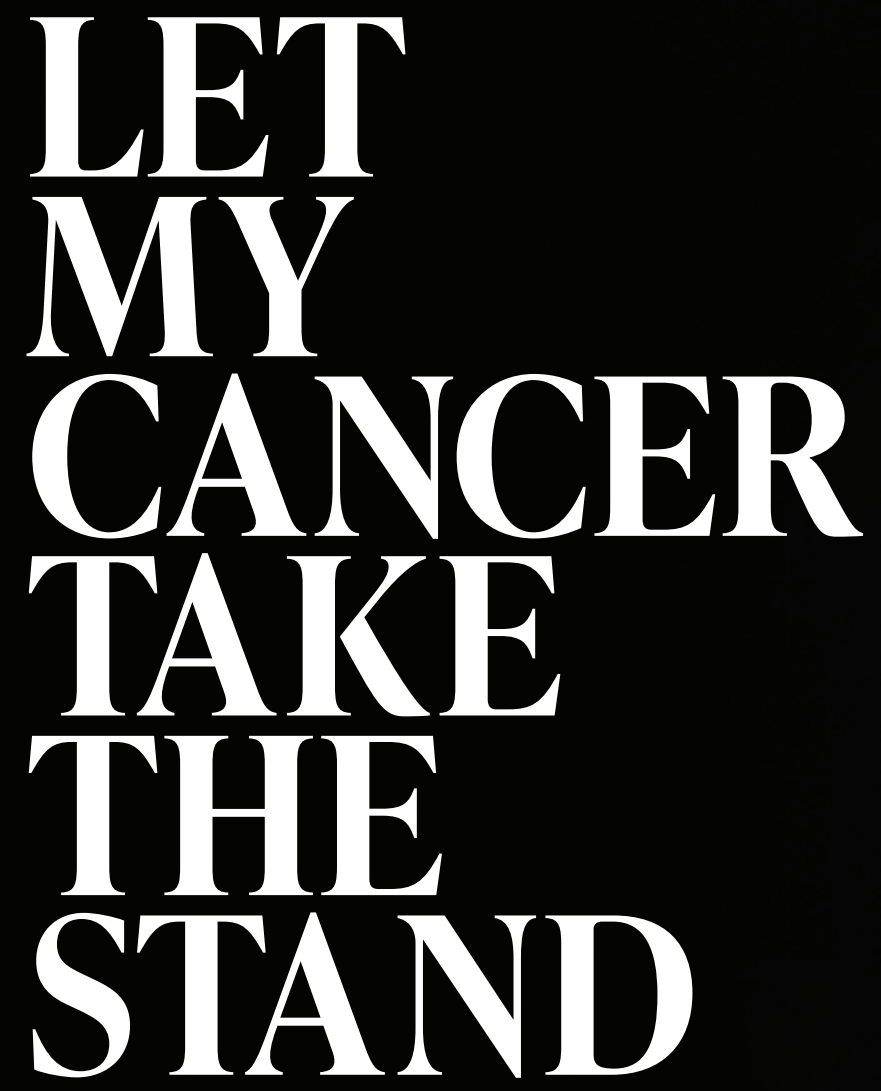

Aniyah, 40

Diagnosed with breast cancer

Cancer doesn't discriminate. So why do our clinical trials?

African Americans make up only 3\% of participants in cancer clinical trials. ${ }^{*}$ Yet they represent $22 \%$ of the total expected incidence of cancer in the United States.

\begin{tabular}{|l|}
\hline \\
THISISTHE \\
TRIAL for \\
\#CLINICAL \\
EQUALITY \\
\hline
\end{tabular}

\section{Demand change at} ClinicalEquality.com

\section{FCBHEALTH}

NETWORK

\footnotetext{
*Percentages of patients calculated from trials supporting FDA drug approvals between July 2008 and June 2018 that documented the inclusion of White, Asian, Black, and Hispanic patients.
}

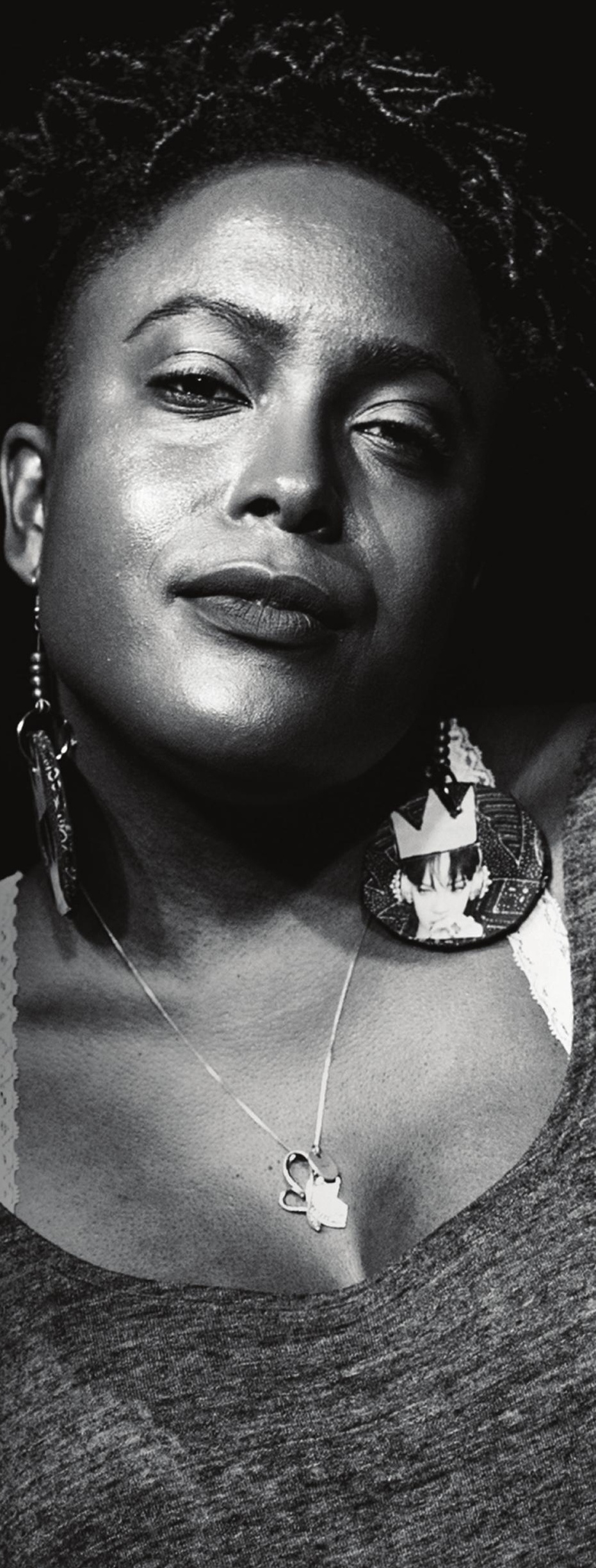




\section{Next-Generation Sequencing for Advanced Pancreatic Cancer: $A$ Technology Whose Time Has NOT Come}

Howard S. Hochster, MD

$\mathrm{n}$ this edition of ONCOLOGY ${ }^{\circledR}$, Christine Y. Chung, DO, and colleagues report on the next-generation sequence (NGS) testing status of 77 patients referred to their practice for phase 1 study enrollment. It seems in the case of both community and academic practices, in their Colorado area referral zone, about half the patients had previously received either tumor or circulating tumor (ct)DNA testing for actionable mutations. Although the authors point out the rate of sequencing is approximately equal in the 2 settings, my question is why physicians are even doing this expensive testing.

Through The Cancer Genome Atlas $^{1}$ determination of molecular profile for 150 pancreatic cancers, we know that $93 \%$ of patients will have KRAS mutations and another 10\% other, usually concurrent RAS mutations. TP53 mutations occur $72 \%$ of the time, and only $4 \%$ to $5 \%$ have a BRCA mutation. Other actionable mutations are really quite rare. For example, $3 \%$ will have $B R A F \mathrm{mu}-$ tations. Also, in the SWOG 1513 study (NCT02890355), , E. Gabriela Chiorean, MD, and her colleagues showed, in a second-line chemotherapy setting, that perhaps $30 \%$ had some kind of DNA repair defective gene based on a defined "HDR" assay, yet the addition of a PARP inhibitor to FOLFIRI (folinic acid, 5-FU, oxaliplatin, and irinotecan) was not beneficial.

In the "Know Your Tumor" (KYT) study $^{3}$ referenced by Chung, the patients who had sequencing-informed treatment seemed to do better. But let's look further at these data. Nearly 1900 patients were enrolled and 1082 were sequenced. Only 667 had outcome data and $38 \%$ of these were considered "actionable," yet many of these are mutations with no known molecular drugs effective for pancreatic cancer, including $A T M$, AKT2, PALB2, and CDK mutations. The outcome data are heavily influenced by the very few with microsatellite instability-high tumors, diagnosable with immunohistochemistry-no NGS required-and NTRK fusions. The outcome of this so-called actionable group was indeed superior, yet what does it represent? This is a noncontrolled trial that perhaps tells us that patients with DNA repair deficits do better overall, perhaps due to sensitivity to our current chemotherapy drugs. It does tell us very little beyond the few actionable mutations we already know about.

Finally, we know that patients with germline mutations may be candidates for olaparib (Lynparza) following induction chemotherapy. However, the guidelines recommend that all patients receive germline testing. This is less expensive than tumor NGS or even ctDNA testing, and may be done even by generally available platforms such as 23andMe. If we perform tumor NGS to find BRCA mutations and then later perform germline testing, this will lead to increased costs to the health care system. Additionally, the approval for use of this PARP inhibitor in pancreatic cancer does not include patients with somatic BRCA mutations.

A new class of RAS inhibitor drugs, specifically targeting KRAS G12C is now in the clinic. These drugs are showing activity in non-small cell lung cancer and potentially will be active in colon cancer. Once these drugs and others targeting more common KRAS mutations have been shown effective for pancreatic cancer, there will be a good reason to perform NGS testing on all patients with pancreatic cancer. At the moment, NGS testing is an expensive test that would cost the health care system over $\$ 200$ million annually, if all 60,000 cases of newly diagnosed pancreatic cancer were tested. Personally, I am looking forward to when this will be a costeffective technology.

\section{REFERENCES}

1. Cancer Genome Atlas Research Network. Integrated genomic characterization of pancreatic ductal adenocarcinoma. Cancer Cell. 2017;32(2):185-203.e13. doi: 10.1016/j. ccell.2017.07.007

2. Chiorean EG, Guthrie KA, Philip $P A$, et al. Randomized phase II study of second-line modified FOLFIRI with PARP inhibitor ABT-888 (Veliparib) (NSC-737664) versus FOLFIRI in metastatic pancreatic cancer (mPC): SWOG S1513. J Clin Oncol. 2019;37(suppl 15):4014. doi:10.1200/ JC0.2019.37.15 suppl.4014

3. Pishvaian M, Blais E, Brody J, et al. Overall survival in patients with pancreatic cancer receiving matched therapies following molecular profiling: a retrospective analysis of the Know Your Tumor registry trial. Lancet Oncol. 2020;21(4):508-518. doi: 10.1016/S1470-2045(20)30074-7 


\section{ARE YOU THINKING DEEP ENOUGH IN RELAPSED OR REFRACTORY MULTIPLE MYELOMA?}

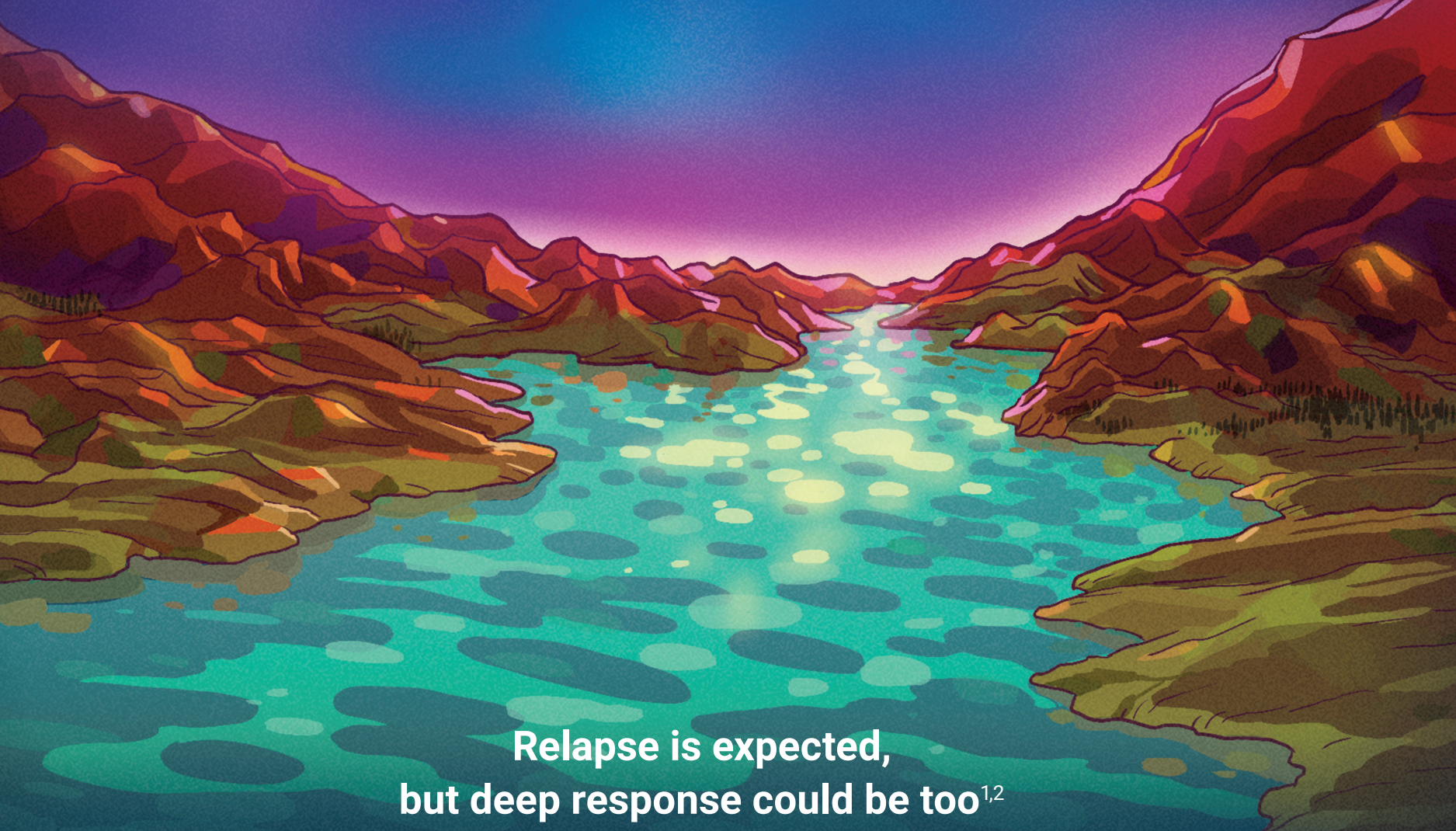

With each relapse, multiple myeloma becomes increasingly difficult to control. As the disease progresses, very few patients (less than $5 \%$ ) experience a deep response ${ }^{1,2}$ However, evidence suggests a deep response may be associated with improved PFS and OS..$^{1-3}$ Therefore, shouldn't a goal of treatment be to achieve a deep response in as many patients as possible?

The hope is that more patients may achieve a deep response with emerging therapies on the horizon.

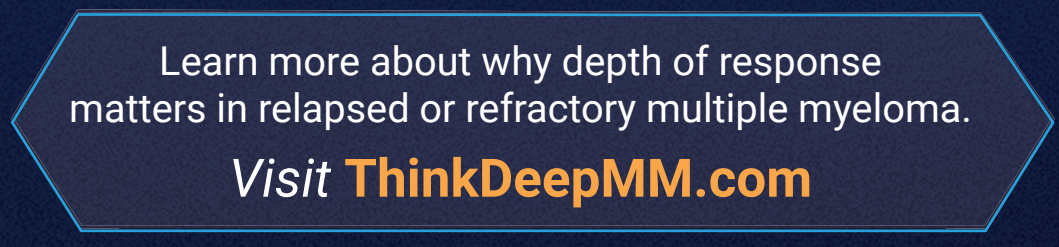

OS=overall survival; PFS=progression-free survival.

References: 1. Kumar SK, Dimopoulos MA, Kastritis E, et al. Natural history of relapsed myeloma, refractory to immunomodulatory drugs and proteasome inhibitors: a multicenter IMWG study. Leukemia. 2017;31(11):2443-2448. 2. Gandhi UH, Cornell RF, Lakshman A, et al. Outcomes of patients with multiple myeloma refractory to CD38-targeted monoclonal antibody therapy. Leukemia. 2019;33(9):2266-2275. 3. Lonial S, Anderson KC. Association of response endpoints with survival outcomes in multiple myeloma. Leukemia. 2014;28(2):258-268.

Janssen Oncology

(C) Janssen Biotech, Inc. 2020

All rights reserved. $11 / 20 \mathrm{cp}-183009 \mathrm{v} 1$

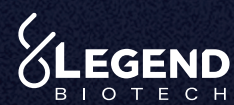

BIOTECH 


\section{MEET OUR EXPERT}

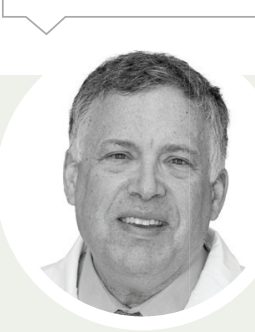

Herbst is Ensign

Professor of Medicine

(medical oncology)

and professor of

pharmacology, chief

of medical oncology,

and associate cancer

center director for

translational science

at Yale Cancer Center

and Smilow Cancer

Hospital, in New Haven,

Connecticut, as well

as cochair of the 22nd

Annual International

Lung Cancer Congress ${ }^{\circledR}$,

hosted by Physicians'

Education Resource,

LLC (PER $\left.{ }^{\circledR}\right)$.

\section{Tracing Steps of Clinical Research in Lung Cancer}

\section{"Using science to guide therapy... has dropped the death rate in lung cancer historically."}

Compared with decades ago, survival times for patients with lung cancer have dramatically increased, thanks to the introduction of targeted therapies and immune checkpoint inhibitors. Since these initial advances, investigators have focused on ways to further these results, by overcoming mechanisms of innate and acquired resistance across modalities and by discovering additional biomarkers of response.

Even as therapies garner increasingly favorable efficacy profiles, it will be imperative to identify the barriers to response that continue to be seen in certain subsets of patients. To discuss the research path that led the field to this point, ONCOLOGY $Y^{\circledR}$ sat down with Roy S. Herbst, MD, PhD, who is Ensign Professor of Medicine (medical oncology) and professor of pharmacology, chief of medical oncology, and associate cancer center director for translational science at Yale Cancer Center and Smilow Cancer Hospital, in New Haven, Connecticut, to shed light on the topic.

Herbst is the program cochair of the 22nd Annual International Lung Cancer Congress ${ }^{\circledR}$, hosted by Physicians' Education
Resource, LLC (PER $\left.{ }^{\circledR}\right)$, to be held July 29 to 31, 2021. The meeting will cover emerging topics in lung cancers and will review continuing trends in the treatment paradigm.

\section{During your career,} become one of the most rapidly progressing disciplines in oncology. What has changed in that time? HERBST: I started in this field in 1994. At that time, I was a fellow at Dana-Farber [Cancer Institute]. What we had was cytotoxic chemotherapy, and it was pretty toxic with little benefit. There was no real plateau [in the survival curves], or no tail on the curve, so to speak.

I had trained in research. Not much progress had [occurred in the field when I started]. We were seeing some effects with bringing chemotherapy to the early stages of disease with radiation, but it was clear we needed to do more.

I started to see patients with lung cancer at Dana-Farber and I got involved with some early clinical trials. Soon thereafter, I was recruited to [The University of Texas] MD Anderson Cancer Center in Houston. [I was involved with] early trials with tyrosine kinase inhibi- tors, against EGFR, the first one being ZD1839 (gefitinib; Iressa). I was very interested in that pathway scientifically, and I became the lead investigator on those trials at MD Anderson. They were open in lung, breast, and prostate cancer, and they worked really well in lung. We saw about 1 in 10 patients responding, and we started to see these drugs were working. We treated patients and we searched for the biomarker. We realized that those patients who had not smoked to date did best and, of course, around 2004, several groups in Boston, using samples from trials that we had actually helped design, found a specific mutation in the EGFR gene in patients who responded well. ${ }^{1}$ The rest is history.

From then on, we saw such great advances in lung cancer with targeted therapy. Then around 2009 to 2010, we saw immunotherapy [emerge]. Using science to guide therapy and bring the therapy from the lab to the clinic has dropped the death rate in lung cancer historically.

Q: What is it like for you to be a part of that kind of revolution? HERBST: We've always treated patients with compassion. 
We've always used multimodality care, and when you can, surgery and radiation with medical oncology, or chemotherapy. We've been able to raise the bar, [but it's] still not nearly far enough. Many patients still die from this disease.

But the fact that we could take scientific discoveries—sometimes with biomarkers, so we can understand who benefits [more] than others, and learning more about the science in the lab first - and [translate that to] targeted therapy and immunotherapy is fantastic, I think. It speaks to how we are bridging the gap and helping people live longer with this disease. That's what gets me up every morning: seeing the science translated to the patient.

Q: In frontline advanced disease, treatment breaks down along 2 tracks: targeted therapy, if a driver mutation is present, or immunotherapy. Will that approach continue, or is there a setting in which immunotherapy would displace targeted therapy?

HERBST: Right now, we look for driver mutations. While the response rates are high [with targeted therapy], the recurrence will occur at some point because of resistance to those drugs. But right now, it's pretty clear that if you have a target, you use the target first, and then immunotherapy is used more in patients who don't have those targets. Many of them are more likely to be smokers, and they have [higher] tumor mutational burden.

That said, the fact that we don't cure anyone with targeted therapy, and [the fact that] these patients do [develop recurrent disease], begs this question: Can we use either standard checkpoint PD-1/PD-L1 or other immunotherapy in these patients to try to have a better effect? These are all things that we're looking at now in the lab and in the clinic.

\section{Q: How do you break through and expand the benefit of} immunotherapy for more patients? HERBST: You have to ask [whether there's] primary resistance or acquired resistance. [An example of] primary resistance is that even with PD-L1-high tumors, [when patients are treated] with pembrolizumab [Keytruda], only $50 \%$ [respond]. Why are those other $50 \%$ negative? We have to look at those $50 \%$ and understand what else is happening. What are the regulatory mechanisms going on even though we see PD-L1 [expression]?

[Then, some patients] initially respond to immunotherapy, and then they become refractory; [in other words], they've responded and then they stopped responding. What's going on? That's the group for whom we have to look at tumor images. Are there new mutations? Have they lost MHC1? We are uncovering resistance mechanisms. What we do is we take those models of those patients' tumors and put them into mice. [When] we have a mouse model of a patient's tumor that became resistant, then we can start to look at innate immune mechanisms or other ways to activate the immune system [in that patient]. That's what keeps me going: trying to find new combos, new immunotherapies that use biology to promote even better therapy.

\section{Q:}

\section{What is the latest thinking} about the best way to use liquid biopsy vs tissue testing?

HERBST: I clearly want tissue testing for diagnosis; I don't think you should make a histology [decision] based on a liquid biopsy. But after that, if [the test is] sensitive enough and assuming enough tumor is being shed, the [liquid] biopsy is a very good way to measure mutational status. If the liquid is negative, I would look to tissue, [the test of] which is going to be more sensitive.

Q: How did you become interested in improving diversity in clinical trials? Why, in your experience, is it so difficult to get patients of color from underserved communities to participate? HERBST: Diversity coordinates with access. We certainly want to make sure everyone has access to clinical trials. I think that by putting someone on a clinical trial, it forces the clinician to think about the standard of care. I think care is improved in settings where there are clinical trials. You [also] want to have experience in those minority populations for the [disease] activity, although I don't believe that in most cases there'll be much difference. You want there to be trust of the community and the drugs. Diversity is important. Diversity is important in the workplace. It's important in clinical trials [and] supported in all aspects of life.

\section{Of all your research, what - results do you think have had the most impact?}

HERBST: My most impactful work is the use of EGFR inhibitors in lung cancer: understanding of their use, of toxicity, the response, bringing those drugs to the clinic, and understanding biomarkers involved in their use. Then, of course, bringing it all the way to phase 3 [trials of osimertinib (Tagrisso) in untreated patients as adjuvant] therapy with highly positive response. Those results were presented at the American Society of Clinical Oncology 2020 Virtual Scientific Program plenary and in the New England Journal of Medicine. ${ }^{2,3}$

FINANCIAL DISCLOSURE: The author has no significant financial interest in or other relationship with the manufacturer of any product or provider of any service mentioned in this article.

\section{REFERENCES}

1. Lynch TJ, Bell DW, Sordella R, et al. Activating mutations in the epidermal growth factor receptor underlying responsiveness of non-small-cell lung cancer to gefitinib. N Engl J Med. 2004;350(21):2129-2139. doi:10.1056/ NEJMoa04093

2. Herbst RS, Tsuboi M, John T, et al. Osimertinib as adjuvant therapy in patients (pts) with stage IB-IIIA EGFR mutation-positive (EGFRm) NSCLC after complete tumor resection: ADAURA. J Clin Oncol. 2020;38(18 suppl 18):abstr LBA5. doi:10.1200/JCO.2020.38.18_suppl. LBA5

3. Wu Y-L, Tsuboi M, He J, et al; ADAURA Investigators. Osimertinib in resected EGFR-mutated non-small-cell lung cancer. N Engl J Med. 2020;383(18):1711-1723. doi:10.1056/NEJMoa2027071 


\section{Molecular Profiling Practices in Pancreatic Adenocarcinoma: Academic vs Community Physicians}

Christine Y. Chung, DO; Rachael Galvin, DO, MPH; Ella Achenbach; Oliwier Dziadkowiec, PhD; and Shiraj Sen, MD, PhD

\begin{abstract}
:
BACKGROUND: Pancreatic adenocarcinoma (PDAC) is relatively rare but highly aggressive, with most patients diagnosed once they have metastatic or locally invasive disease. Molecular profiling is being explored as a tool for selecting patients for targeted therapy clinical trials and for assessing whether targeted therapies may be effective in PDAC. Whether molecular profiling is being performed at both academic and community oncology clinics has yet to be examined. Here, we characterized the molecular profiling practice patterns in patients with PDAC in academic versus community practices in Denver, Colorado.
\end{abstract}

METHODS: We retrospectively reviewed records of all patients with refractory, metastatic PDAC who were referred to a tertiary clinical trials drug development unit in Denver between 2014 and 2019.

RESULTS: Of 77 patients, 41 (55\%) were men with a mean age of 65 years (SD, 9.3). Fifty-three patients (69\%) were referred from the community and $20(26 \%)$ from academic centers; 4 (5\%) were self-referred. A total of 51\% received profiling prior to referral; 29 of $50(58 \%)$ were from the community and 10 of 21 (47\%) from academic settings. Guardant was the most commonly ordered test ( 47 of 77;61\%); FoundationOne was the second most common (40 of 77; 52\%). Twenty-three of 77 patients (30\%) received both Guardant and FoundationOne testing, and 3 of 77 (4\%) received Caris MI Profile. One patient received a Mocha assay and another received Ascend/Clarient fluorescence in situ hybridization (FISH). Four patients were self-referred, 2 of whom underwent both Guardant and FoundationOne, 1 who underwent Guardant testing only, and 1 who did not receive any molecular profiling testing.

CONCLUSIONS: This study characterizes molecular profiling practice patterns in individuals with advanced PDAC who were referred to a tertiary clinical trials drug development unit. Both academic and community physicians were found to order profiling about $50 \%$ of the time. Further research is needed to determine impact on clinical trial enrollment and detection of PDAC.

KEYWORDS: molecular profiling, pancreatic adenocarcinoma, ctDNA

\section{Introduction}

Pancreatic adenocarcinoma (PDAC) is the fourth leading cause of cancer death in the United States ${ }^{1}$ with a 5 -year overall survival $(\mathrm{OS})$ rate around $8 \%$ in the United States, ${ }^{2}$ despite years of research and therapeutic development. Current treatment of PDAC is mostly focused on surgical resection for cure. In cases of unresectable or metastatic PDAC, however, chemotherapy has been the only other treatment option outside clinical trials until recently.
In the last 15 -to 20 years, a large body of transcriptomic and immunophenotypic research has led to the development of numerous targeted therapy-based and immunotherapy-based clinical trial options; subsequently, the first FDA approval of a targeted therapy in advanced PDAC occurred in 2005. With the recent approval of olaparib (Lynparza) for the maintenance treatment of adult patients with germline BRCA-mutated metastatic PDAC, pancreatic cancer treatment has begun to transition from a one-size-fitsall approach to a more precise approach based on genetic variants. ${ }^{3}$

While tissue-based assays such as FoundationOne $\mathrm{CDx}$ remain the mainstay for molecularly characterizing PDAC, newer blood-based platforms have begun to emerge. Guardant360, for instance, molecularly profiles tumors based on circulating tumor DNA that is released into the blood via apoptosis, necrosis, and active secretion. ${ }^{3}$ Guardant360 can 
detect EGFR mutations in non-small cell lung cancer (NSCLC); consequently, it became the first FDA-approved liquid biopsy companion diagnostic that uses next-generation sequencing (NGS). The role of molecular profiling in PDAC remains under investigation.

In a retrospective analysis of 1856 patients with pancreatic cancer who were referred to the Know Your Tumor (KYT) program, ${ }^{4} 1082$ (58\%) received personalized reports based on molecular testing results. Only 189 patients had actionable molecular alterations and underwent matched therapies, but these treatments were associated with a significantly longer median OS than that of patients who received only unmatched therapies. ${ }^{4}$ More recently, the POLO trial $^{5}$ (NCT02184195) evaluated the efficacy of olaparib as maintenance therapy in patients with a germline BRCA1 or BRCA2 mutation and metastatic pancreatic cancer who had not progressed during first-line platinum-based chemotherapy. The results revealed that progression-free survival was significantly longer in the olaparib group, leading to the first FDA approval for a targeted therapy in BRCA-mutated PDAC with a PARP inhibitor. Because it is FDA approved for all solid tumors, pembrolizumab (Keytruda) is FDA approved for PDAC specifically, disease with microsatellite instability (MSI) tumors and for disease with high tumor mutation burden. ${ }^{6}$ Numerous other active studies are examining potential molecular targets of PDAC.

In the past, molecular profiling has been shown to be underutilized in community-based practices. In one study, 292 community oncologists completed a questionnaire to assess how well they understood this technology; about 69\% either could not define what molecular profiling was, or answered incorrectly. ${ }^{7}$ The investigators also found that community-based oncologists use molecular profiling far less frequently than do academic-based oncologists (59 of 292 community oncologists of whom were polled

\section{TABLE 1. Patient Demographics}

\begin{tabular}{|c|c|}
\hline \multirow[t]{2}{*}{ Patient characteristic } & Total $(n=77)$ \\
\hline & n (\%) \\
\hline Age in years, mean (SD) & $65(9.3)$ \\
\hline \multicolumn{2}{|l|}{ Gender } \\
\hline Female & $36(45)$ \\
\hline Male & $41(55)$ \\
\hline \multicolumn{2}{|l|}{ Initial stage } \\
\hline IA & $3(3.9)$ \\
\hline IB & $3(3.9)$ \\
\hline IIA & $4(5.2)$ \\
\hline IIB & $13(16.9)$ \\
\hline III & $5(6.5)$ \\
\hline IV & 37 (48) \\
\hline Unknown & $13(16.9)$ \\
\hline Comorbidities, median (range) & $2(0-7)$ \\
\hline Previous surgery & $18(23.4)$ \\
\hline Pancreatoduodenectomy & $13(16.9)$ \\
\hline Distal pancreatectomy & $1(1.3)$ \\
\hline Segmental pancreatectomy & $1(1.3)$ \\
\hline Total pancreatectomy & $1(1.3)$ \\
\hline Other & $2(2.6)$ \\
\hline No surgery & $59(76.6)$ \\
\hline \multicolumn{2}{|l|}{ Prior chemotherapy regimen } \\
\hline Gemcitabine/abraxane & $64(83.1)$ \\
\hline FOLFIRINOX & $55(71.4)$ \\
\hline $5-\mathrm{FU}$ & $14(18.2)$ \\
\hline Xeloda & $10(13)$ \\
\hline
\end{tabular}

on use of these tests). For example, 33\% of community oncologists vs $74 \%$ of academic oncologists said they use molecular profiling in treating their patients with lung cancer. ${ }^{7}$

The aim of our study was to evaluate whether there are differences in molecular profiling practice patterns based on treatment site for individuals with PDAC, a question that has not yet been addressed in the literature. Here, we examined the practice patterns of academic vs community physicians in relation to molecular profiling practices and PDAC in Denver, Colorado.

\section{Methods}

We performed a retrospective review of 77 patients with refractory, metastatic PDAC who were referred between August 2014 and May 2019 to the Sarah Cannon Research Institute (SCRI) at HealthONE, an oncology drug development unit in Denver. These patients all had refractory disease after surgical intervention, chemotherapy, and/or radiation therapy. All patients with metastatic PDAC who were referred to SCRI in this period were included.

Patients with PDAC were referred by 31 community physicians from 12 different community-based practices and by 
12 academic physicians from 4 different academic medical centers (Table $\mathbf{1}$ ). As a proxy for identifying academic vs nonacademic practices, we classified each referring oncologist based on whether they are affiliated with a National Cancer Institute-designated cancer center.

All available data were entered into a Microsoft Excel database (Microsoft Corporation, 2016). A univariate analysis was performed to compare patients who were referred to Sarah Cannon Research Institute (SCRI) from community vs academic medical centers. Patients who "self-referred" ( $n=4)$ were not included in the univariate analysis. This analysis was completed using R Studio (Team, R, 2015). Fisher exact and $\chi 2$ tests were used to assess categorical variables; the $t$ test was used for continuous variables. Statistical significance was set at $P<.05$. This research was approved by the HealthOne HCA institutional review board.

\section{Results}

A total of 41 of 77 patients $(55 \%)$ were men; the mean age of the cohort was 65 years (Table 1 ). Patients had between 0 and 7 medical comorbidities. Functional status was determined by ECOG performance score (PS), which ranged from 0 to 3; $53 \%$ of patients had an ECOG PS of 1. Additionally, 28 of 77 patients (39\%) were smokers; 41 (57\%) were not and 3 had unknown status (4\%). Initial staging varied from stage I to stage IV, and 37 of $77(48 \%)$ were stage IV when referred to our facility. As seen in Table 1, surgical intervention prior to referral occurred in 18 of 77 patients $(23 \%)$; the surgeries including 13 pancreatoduodenectomies, 1 distal pancreatectomy, 1 segmental pancreatectomy, and 1 total pancreatectomy. Almost all patients $(97 \% ; 75 / 77)$ underwent chemotherapy prior to referral, which consisted of either gemcitabine and nab-paclitaxel (Abraxane); FOLFIRINOX (folinic acid, fluorouracil, irinotecan, oxaliplatin); fluorouracil; or a combination of these. Eleven patients (14\%) participated in some treatment portion of a clinical trial

TABle 1 CONTINUED. Patient Demographics

\begin{tabular}{|c|c|}
\hline \multirow[t]{2}{*}{ Patient characteristic } & Total $(n=77)$ \\
\hline & n (\%) \\
\hline \multicolumn{2}{|l|}{ On trial prior to referral } \\
\hline$Y_{e s}{ }^{a}$ & $11(14.3)$ \\
\hline No & 66 (85.7) \\
\hline Patients receiving radiation & $22(28.6)$ \\
\hline ECOG 0 & $7(9.1)$ \\
\hline ECOG 1 & $53(68.8)$ \\
\hline ECOG 2 & $6(7.8)$ \\
\hline ECOG 3 & $4(5.2)$ \\
\hline ECOG unknown & $6(7.8)$ \\
\hline \multicolumn{2}{|l|}{ Smoking status } \\
\hline Current & $28(38.9)$ \\
\hline Nonsmoker & $41(56.9)$ \\
\hline Unknown & $3(4.2)$ \\
\hline \multicolumn{2}{|l|}{ Patient referral source } \\
\hline Academic medical center & $20(26)$ \\
\hline Community medical center & $53(68.8)$ \\
\hline Self-referred & $6(7.8)$ \\
\hline \multicolumn{2}{|l|}{ Physician location } \\
\hline Academic medical center & 12 \\
\hline Community medical center & 31 \\
\hline \multicolumn{2}{|l|}{ Center type } \\
\hline Academic medical center & 4 \\
\hline Community medical center & 12 \\
\hline
\end{tabular}

5-FU, fluorouracil; FOLFIRINOX, folinic acid, fluorouracil, irinotecan, oxaliplatin.

aTrials: nivolumab/ulocuplumab (1), capecitabine+/-ruxolitinib (1), TH302 hypoxia-inducing prodrug conjugate) (1), SBP-101 (polyamine inhibitor) (1), unknown (1), 15-1613 Bayer PTEFb (CDK9i) (1), Trial 15-1135: MLN0128 + alisertib (1), OncoMed M18-006 demcizumab and gemcitabine/abraxane, AM0010 + FOLFOX (1), phase 1 trial TAK-931 (CDC7 inhibitor) (1), phase 1 OMP52M51, notch 1 Rc inhibitor (1), phase 1 Kinex oral paclitaxel (1), Protocol BBI503 stemness kinase inhibitor, phase 1 trial FF-10502

prior to referral and 22 received radiation.

Fifty-three of the 77 patients $(69 \%)$ were referred from the community and $20(26 \%)$ from academic centers; $4(5 \%)$ were self-referred. A total of $51 \%$ received profiling prior to referral; 29 of 50 (58\%) were from the community and 10 of 21 (47\%) from academic settings. Guardant was the most commonly ordered test (47 of $77 ; 61 \%)$; FoundationOne was the second most common (40 of $77 ; 52 \%$ ). Twentythree of 77 patients $(30 \%)$ received both Guardant and FoundationOne testing, and
3 of 77 (4\%) received Caris MI Profile. One patient received a Mocha assay and another received Ascend/Clarient fluorescence in situ hybridization (FISH). Four patients were self-referred, 2 of whom underwent both Guardant and FoundationOne, 1 who underwent Guardant testing only, and 1 who did not receive any molecular profiling testing. All blood-based molecular tests were ordered between 2013 and 2019.

When we compared patients from community and academic medical centers, we found them to be of similar age, with 
TABLE 2. Univariate Differences of Characteristics Between Patients Referred From Academic vs Community Medical Centers

\begin{tabular}{|c|c|c|c|}
\hline \multirow[t]{2}{*}{ Characteristic } & \multicolumn{2}{|l|}{ Referral source } & \multirow[t]{2}{*}{$P$} \\
\hline & $\begin{array}{l}\text { Academic MC } \\
(n=20 ; 26.0 \%)\end{array}$ & $\begin{array}{l}\text { Community MC } \\
(n=52 ; 67.5 \%)\end{array}$ & \\
\hline Age in years, mean (SD) & $66(8.3)$ & $64(8.1)$ & $.49^{\mathrm{a}}$ \\
\hline \multicolumn{4}{|l|}{ Gender } \\
\hline Female & $7(35)$ & $28(53.8)$ & $.24^{\mathbf{b}}$ \\
\hline Male & $13(65)$ & $24(46.2)$ & \\
\hline \multicolumn{4}{|l|}{ Initial stage } \\
\hline IA & $0(0)$ & $3(5.8)$ & $.55^{\mathrm{c}}$ \\
\hline IB & $0(0)$ & $2(3.8)$ & $1.0^{\mathrm{c}}$ \\
\hline IIA & $1(5)$ & $3(5.8)$ & $1.0^{\mathrm{c}}$ \\
\hline IIB & $2(10)$ & $8(15.3)$ & $0.72^{\mathrm{c}}$ \\
\hline III & $1(5)$ & $4(7.7)$ & $1.0^{\mathrm{c}}$ \\
\hline IV & $10(50)$ & $26(50)$ & $1.0^{\mathrm{b}}$ \\
\hline Unknown & $6(30)$ & $6(11.5)$ & $.08^{\mathrm{c}}$ \\
\hline Underwent surgery & $18(69)$ & $21(75)$ & \\
\hline Receiving chemotherapy & 19 (95) & $51(98.1)$ & $.48^{\mathrm{c}}$ \\
\hline Gemcitabine/abraxane & $18(90)$ & $44(84.6)$ & $.72^{\mathrm{c}}$ \\
\hline FOLFIRINOX & $17(85)$ & $36(69)$ & $.24^{\mathrm{c}}$ \\
\hline 5-FU & $3(11)$ & $11(21)$ & $.74^{\mathrm{c}}$ \\
\hline Xeloda & $1(5)$ & $9(17)$ & $.26^{\mathrm{c}}$ \\
\hline Received radiation & $6(30)$ & $14(26.9)$ & $.77^{\mathrm{c}}$ \\
\hline On trial prior to presentation & $5(25)$ & $6(11.5)$ & $.16^{\mathrm{c}}$ \\
\hline ECOG 0 & $3(15)$ & $3(5.8)$ & $.26^{\mathrm{c}}$ \\
\hline ECOG 1 & $15(75)$ & 35 (67.3) & $.58^{\mathrm{c}}$ \\
\hline ECOG 2 & $1(5)$ & $3(5.8)$ & $1.0^{\mathrm{c}}$ \\
\hline ECOG 3 & $0(0)$ & $4(7.7)$ & $.57^{\mathrm{c}}$ \\
\hline ECOG unknown & $1(5)$ & 7 (13.5) & $.43^{\mathrm{c}}$ \\
\hline Current smoker & $7(35)$ & $1.93(1.30)$ & $.79^{\mathrm{c}}$ \\
\hline
\end{tabular}

5-FU, fluorouracil; FOLFIRINOX, folinic acid, fluorouracil, irinotecan, oxaliplatin; MC, medical center. ${ }^{a}$ t test

${ }^{b} x 2$

'Fisher exact

more male patients being referred from the academic than community settings $(65 \%$ vs $46 \%$ ) and more individuals with initial stage being unknown being referred from the academic than community settings (30\% vs $11 \%$ ), although these differences were not statistically significant (Table 2). We also found that a higher percentage of patients referred from academic medical centers $(25 \%)$ than community medical centers clinical trials drug development unit in Denver. Both academic and community physicians were found to order profiling about $50 \%$ of the time. Given that bloodbased molecular profiling has become more widely available and supported by more generous patient financial assistance programs only in recent years, it is possible that its use may overtake tissue-based testing in the future. It was just in the past year (August 2020) that Guardant360, a blood-based test, was approved by the FDA as the first liquid biopsy NGS companion diagnostic test for solid tumors. ${ }^{8}$ All bloodbased molecular tests in our study were ordered between 2013 and 2019. During this time, only 40 tissue-based molecular profiling tests were ordered, fewer than the 47 blood-based molecular profiling tests ordered. Moreover, given our understanding of tumor heterogeneity and challenges of limited biopsy samples in advanced PDAC, it remains important to study and validate how concordant or discordant these testing modalities are. Interestingly, blood-based testing was ordered in addition to tissue-based testing in 30\% of patients in our analysis. As more patients receive both types of testing, the results may provide insight into answering questions regarding concordance. Ongoing trials are also exploring whether there may be some benefit to ordering repeat molecular profiling at various lines of therapy in order to best understand mechanisms of resistance to anticancer therapies.

Historically, a lack of insurance coverage has often been cited as a barrier to obtaining molecular profiling of an individual's tumor. Recently, however, CMS has proposed national coverage of diagnostic tests that use NGS technology in patients with recurrent or metastatic solid cancers who have not previously used the test and who wish to pursue further treatment. ${ }^{9}$ Additionally, the FDA has approved tissueand blood-based testing as general tumor profiling or as companion diagnostic tests in all solid tumors (but not blood cancers). Specifically, testing is approved in PDAC for BRCA1/2; in NSCLC for EGFR, ALK, 
and $B R A F$; in breast cancer for $E R B B 2$; and in colorectal cancer for KRAS and NRAS. ${ }^{10}$ In our analysis, insurance was a factor in only 1 case in which the provider ordered the test; testing was not performed due to pending insurance approval.

Our study had the notable finding that community and academic oncologists ordered molecular profiling at the same rate-about $50 \%$ of the time-a result that is contrary to previous data. As more targeted therapy clinical trials move into the community setting and we continue to see more US FDA approvals for targeted therapies every year, it is possible that community oncologists will continue to perform molecular profiling with the same frequency as academic oncologists.

As mentioned previously, the KYT trial ${ }^{4}$ was a retrospective study in which patients received personalized reports based on molecular testing results. It was the first step in attempting to understand the application of molecular profiling in PDAC. Only 189 patients had actionable molecular alterations and underwent matched therapies, but these treatments were associated with a significantly longer median OS than that of patients who received only unmatched therapies. Although the KYT trial results showed promising, positive results, multiple potential confounding factors may have affected them. For example, the matched treatment may be effective in prolonging survival itself, or it could be that the presence of an actionable mutation and/or the ability to receive matched treatment might in themselves be a good prognostic factor for better outcomes. Also, the selection of patients who survived long enough to receive 2 different therapies introduced a selection bias toward those individuals with more indolent disease and better prognosis. Additionally, matched therapies were often given in conjunction with chemotherapy, which poses a challenge in attributing the contribution of parts of each therapy to any clinical benefits seen. Moreover, it should be noted that although the results of the KYT trial seemed promising, the adoption of precision medicine for patients with PDAC had no effect on survival for $97.5 \%$ of patients. For the $2.5 \%$ who could receive a matched treatment, further research by way of randomized clinical trials is warranted to better understand the role of molecular profiling in PDAC. ${ }^{11}$ Continuing to perform molecular profiling on PDAC tumors will be necessary in order to conduct these clinical trials. Given that less than 10\% of those with advanced cancers may have actionable mutations ${ }^{12}$ as determined by currently available commercial assays, further research is also needed in the discovery of novel targets in PDAC.

Several recent tumor-agnostic approvals have also paved the way for targeted therapy in advanced PDAC. For example, 2 tropomyosin receptor kinase inhibitorslarotrectinib (Vitrakvi) and entrectinib (Rozlytrek)—have recently been FDA approved for NTRK fusion-positive cancers, having demonstrated response rates greater than $75 \%$ independent of tumor histology. ${ }^{13}$ Closely analyzing the real-world data of outcomes with these targeted therapies in PDAC will be critical to understanding their efficacy in this specific indication.

When we compared patients referred for early-phase clinical trials from community and academic medical centers, we found no statistically significant differences in the age, gender, stage at diagnosis, prior treatments, or ECOG PS between the 2 groups (Table 2). Thus, we do not believe there was a clinically relevant confounding variable related to these characteristics that may have influenced why there were no differences in rate of molecular profiling testing.

To date, it is not entirely clear what the correct setting and application of molecular profiling in PDAC is. Continued enrollment into clinical trials will be necessary to answer this question. Similarly, performing molecular profiling on PDAC is needed to generate real-world data on the efficacy of targeted therapies that have been approved in a tumor-agnostic manner and to help identify potential new treatments. Outside of a clinical trial, randomized controlled trials would need to be conducted in order to determine whether molecular profiling in metastatic PDAC is cost-effective and whether it improves survival. Such answers are important, because private and public payers will have to determine how much information is required to establish clinical utility and, subsequently, the tier at which molecular testing continues to be covered.

A main limitation to this study is its retrospective nature and the small sample size of 77 patients. It was also a single-center study, in which most patients were from Colorado. Additionally, only $25 \%$ of physicians were from academic institutions, which may reveal inherent bias. Lastly, as all of these patients were referred to our center for clinical trial consideration, they represent only a subset of individuals with PDAC and may be individuals who do not represent the entire population in terms of willingness to receive molecular profiling. The driving factors that underlie why a select group of community oncologists send for molecular profiling and refer patients to a tertiary clinical trials center are unclear at this time. These oncologists may represent a more select group of community physicians who are more inclined to be leaders in molecular-based testing and treatments.

\section{Conclusions}

This study analyzes molecular profiling practices in community vs academic centers in Denver, Colorado. Our study demonstrated that community- and academic-based oncologists both ordered molecular profiling in patients with PDAC about $50 \%$ of the time. This is contrary to prior data suggesting that academic centers perform molecular profiling more often. Further research is needed to determine how molecular profiling practices may impact treatment selection, clinical trial enrollment, and OS.

FINANCIAL DISCLOSURE: The authors have no significant financial interest in or other relationship with the manufacturer of any product or provider of any service mentioned in this article.

For full reference list, visit cancernetwork.com/Chung 5.21 


\title{
Novel Therapies for Metastatic Triple-Negative Breast Cancer: Spotlight on Immunotherapy and Antibody-Drug Conjugates
}

Aiko Nagayama, MD, PhD; Neelima Vidula, MD; and Aditya Bardia, MD, MPH

\begin{abstract}
:
BACKGROUND: Triple-negative breast cancer (TNBC) is a biologically heterogeneous disease that is often associated with worse outcomes compared with other subtypes such as hormone receptor-positive tumors and HER2-positive tumors. While chemotherapy remains the mainstay of standard therapy for metastatic TNBC (mTNBC), several novel treatments have been developed over the past few years. In this review article, we review the major developments in the management of patients with mTNBC.
\end{abstract}

SUMMARY: The combination of chemotherapy and immunotherapy is a potential therapeutic option for PD-L1-positive mTNBC, as the FDA recently approved atezolizumab (Tecentriq) and pembrolizumab (Keytruda) in combination with chemotherapy. Also, 2 targeted therapies-olaparib (Lynparza) and talazoparib (Talzenna)-are FDA approved for the management of mTNBC with germline BRCA mutations, and sacituzumab govitecan, an anti-Trop2 antibody-drug conjugate $(A D C)$, was recently approved for previously treated mTNBC. A number of promising therapies are on the horizon, including AKT inhibitors for PI3K-altered TNBC as well as other ADCs.

KEY MESSAGE: The successful clinical development of immunotherapies, PARP inhibitors, and ADCs for the management of mTNBC has improved the survival outcome of patients. Over the coming years, the therapeutic developments in precision medicine will likely change the mTNBC landscape, and might make the current definition of TNBC as breast cancer that is estrogen receptor negative, progesterone receptor negative, and HER2 negative obsolete.

\section{Introduction}

Triple-negative breast cancer (TNBC) is a heterogenous disease that essentially is a diagnosis of receptor exclusion, referring to tumors that lack hormone receptors and lack of HER2 amplification. Clinically, patients with TNBC, as compared with other breast cancer subtypes, have a higher risk of recurrence and poor prognosis. ${ }^{1}$ Better treatments for TNBC represent a major unmet clinical need in the field of breast oncology, and over the past few years, a number of attempts have been made to change the natural history of this biologically aggressive disease.

One of the major breakthroughs for TNBC has come from a better understanding of the molecular subtypes of TNBC. Recent genomic, transcriptomic, proteomic, and epigenomic studies have shed light on the multilayered heterogeneity of TNBC tumors (Table 1). In one of the first descriptions of the heterogeneity of TNBC, Lehmann et al reported that there are at least 6 subtypes of TNBC: basal-like 1 (BL1), basal-like 2 (BL2), immunomodulatory (IM), mesenchymal (M), mesenchymal stem-like, and luminal androgen receptor (LAR), with distinct gene expression profiles. ${ }^{2}$ Each of these molecular subtypes displays different but overlapping clinicopathological characteristics, heterogenous mutational profiles, and genomic instability. ${ }^{3}$ This classification was later refined to 4 subtypes-BL1, BL2, M, and LAR-by excluding the contribution from tumor-infiltrating lymphocytes (TILs) and stromal cells. ${ }^{4} \mathrm{~A}$ recent study of whole genome sequencing 
of TNBC tumors also confirmed that $59 \%$ were predicted to be homologous recombination repair deficiency (HRD) high, mainly by $B R C A 1 / 2$ deficiency, and HRD-high patients demonstrated better prognosis whereas HRD-low patients, some with mismatch repair deficiency and PIK3CA/AKT1 pathway alterations, demonstrated worse prognosis. ${ }^{5}$ On the protein level, quantitative analysis of TNBC cell lines and breast tumors again showed diverse expression of signaling and biological pathways. ${ }^{6}$ Furthermore, some of the hormone receptor-positive tumors can lose the hormone receptors under pressure from endocrine therapy and evolve into acquired TNBC with a distinct genomic profile. ${ }^{7,8}$ Collectively, TNBC tumors display considerable intratumoral heterogeneity, highlighting the potential value of single-cell sequencing over bulk RNA sequencing in understanding the biology of TNBC. ${ }^{9}$

In terms of treatment targets in TNBC tumors, traditional chemotherapies, such as anthracycline, taxanes, and platinum agents, have been the mainstay of earlyline management of mTNBC. Apart from cytotoxic chemotherapy, other treatment strategies and actionable targets such as PARP inhibition, immunotherapy, AKT inhibition, and antibody-drug conjugates (ADCs) in mTNBC are quickly drawing attention. In this review article, we discuss the major developments of targeted therapies and immunotherapies in the management of patients with mTNBC.

\section{PARP Inhibitors for BRCA- Mutated TNBC}

The DNA damage response process involves sensing and responding to DNA damage, mediating DNA repair, cell cycle regulation, replication stress response, and apoptosis. ${ }^{10}$ The deficiency of DNA damage response harbors genomic instability in cells, but it also provides therapeutic targets, particularly to DNA-damage agents. Carriers of deleterious heterozygous germline mutations in the BRCA1 and $B R C A 2$ genes demonstrate the deficiency of homologous recombination repair, a conservative process of repairing double strand DNA breaks. PARP inhibitors induce apoptosis in $B R C A$-deficient tumor cells by mediating stalled replication forks and double-strand DNA breaks. ${ }^{11}$ For patients with germline BRCA1/2 mutations, PARP inhibitors, including olaparib (Lynparza) and talazoparib (Talzenna), are FDA approved as targeted therapies.

The efficacy and safety of olaparib was demonstrated in the randomized, open-label, phase 3 OlympiAD trial (NCT02000622) conducted in patients with metastatic HER2-negative breast cancer with germline $B R C A$ mutations. Between April 2014 and November 2015, 205 participants were assigned to receive olaparib and 97 to receive standard therapy with single-agent chemotherapy of physician's choice (capecitabine, eribulin, or vinorelbine). The results demonstrated a significant improvement in median progression-free survival (PFS) with olaparib (7.0 vs 4.2 months; HR, $0.58 ; P<.001$ ) with fewer grade 3 and above adverse events (AEs) in the olaparib arm. The response rate was $59.9 \%$ in the olaparib group and $28.8 \%$ in the standard-therapy group. ${ }^{12}$ The final overall survival (OS) analysis demonstrated no statistically significant improvement (19.3 vs 17.1 months; HR, $0.90 ; P=.513) \cdot{ }^{13}$ In the TNBC subgroup, PFS favored olaparib (HR, 0.43, 95\% CI, 0.29-0.63) but the median OS was not statistically different between the 2 groups (17.4 vs 14.9 months; HR, 0.93; $P=.51$ ). Similarly, another PARP inhibitor, talazoparib, was investigated in a randomized, open-label, phase 3 EMBRACA trial (NCT01945775) in patients with advanced breast cancer and germline BRCA1/2 mutations. ${ }^{14}$ Between October 2013 and April 2017, 287 patients were assigned to receive talazoparib and 144 to standard therapy (capecitabine, eribulin, gemcitabine, or vinorelbine). Median PFS was significantly improved in the talazoparib group compared with the standard therapy group
(8.6 vs 5.6 months; HR, $0.54 ; P<.001$ ) with manageable toxicities. ${ }^{15}$ Again, the OS analysis demonstrated no statistically significant improvement (19.3 vs 19.5 months; HR, $0.848 ; P=.17$ ) in the entire cohort. ${ }^{16}$ In TNBC subgroup (130 patients in the talazoparib arm and 60 patients in the standard-therapy arm), PFS favored talazoparib (5.8 vs 2.9 months; HR, 0.60 ; $95 \%$ CI, 0.41-0.87; $P=.0058) .{ }^{16}$

Putting together these data, PARP inhibitors for patients with germline $B R C A 1 / 2$ mutation improved clinically meaningful PFS with manageable toxicity. The potential utility of PARP inhibitors in TNBC is being explored in patients with early-stage breast cancer in the neoadjuvant ${ }^{17}$ and adjuvant settings, ${ }^{18}$ in overcoming resistance by combination therapy, ${ }^{19}$ and in wider patient selection with HRD phenotype beyond germline $B R C A$ mutations. ${ }^{20}$ Notably, a single agent, olaparib, achieved an $82 \%$ response rate in 11 patients with germline PALB2 mutation in the recent proof-of-principle study. ${ }^{21}$ The comparison of efficacy and safety of a PARP inhibitor vs platinum-based treatment in patients with TNBC and germline $B R C A 1 / 2$ mutation has not yet been done.

\section{Immunotherapy for mTNBC}

In the past decade, considerable evidence has been uncovered showing that tumors acquire an immune escape system to facilitate tumor growth by usurping the PD-1/PD-L1 axis between TILs and tumor cells. ${ }^{22}$ Also, inhibiting PD-L1 can induce antitumor activity. ${ }^{23}$

The pivotal IMpassion 130 (NCT02425891) phase 3 trial investigated a selective PD-L1 inhibitor, atezolizumab (Tecentriq), plus nab-paclitaxel (Abraxane) vs placebo plus nab-paclitaxel in patients with mTNBC. From June 2015 through May 2017, a total of 451 patients were randomized on the study. Patients receiving atezolizumab plus nab-paclitaxel had a statistically significant but clinically moderate improvement in PFS in the PD-L1-positive subgroup 
TABLE 1. Key Studies Reporting Genetic and Transcriptomic Classifications in Early Triple-Negative Breast Cancer

\begin{tabular}{|c|c|c|c|c|c|c|}
\hline Classifications & \multicolumn{6}{|c|}{ Triple-negative breast cancer } \\
\hline IHC/FISH & \multicolumn{6}{|c|}{ Lack of ER, PgR, and HER2 } \\
\hline \multirow[b]{2}{*}{ Lehmann subtype $e^{2,4}$} & BL1 & BL2 & IM & M & MSL & LAR \\
\hline & $\begin{array}{l}\text { DNA-damage } \\
\text { response, TP53 } \\
\text { mutation }\end{array}$ & $\begin{array}{l}\text { Growth factor } \\
\text { signaling, metabolic } \\
\text { pathway }\end{array}$ & $\begin{array}{l}\text { Immune signatures, } \\
\text { checkpoint inhibitor } \\
\text { genes }\end{array}$ & $\begin{array}{l}\text { Cell motility, } \\
\text { differentiation, } \\
\text { EMT }\end{array}$ & Angiogenesis & $\begin{array}{l}\text { Luminal gene } \\
\text { expression, AR } \\
\text { expression }\end{array}$ \\
\hline & BLIA & BLIS & \multicolumn{3}{|c|}{ MES } & LAR \\
\hline RNA/DNA profiling ${ }^{60}$ & $\begin{array}{l}\text { STAT signal } \\
\text { transduction } \\
\text { molecules } \\
\text { and cytokines, } \\
\text { overexpression of } \\
\text { CTLA4 }\end{array}$ & $\begin{array}{l}\text { Downregulation of } \\
\text { immune-regulating } \\
\text { pathways }\end{array}$ & \multicolumn{3}{|c|}{ Cell cycle, mismatch repair, DNA damage networks } & $\begin{array}{l}\text { AR, ER, prolactin, } \\
\text { HER4, focal gains on } \\
11 q 13 \text { (CCND1, FGF } \\
\text { family) }\end{array}$ \\
\hline \multirow[b]{2}{*}{ WGS $^{5}$} & \multicolumn{3}{|c|}{ HRD-high } & \multicolumn{3}{|c|}{ HRD-low } \\
\hline & \multicolumn{3}{|c|}{$\begin{array}{l}\text { Germline/somatic mutations of } B R C A 1 / 2 \text {; hypermethylation of } B R C A 1 \\
\text { and } R A D 51 C \text { or loss of } P A L B 2 \text { and } P T E N\end{array}$} & \multicolumn{3}{|c|}{ Mismatch repair deficiency; mutations of $P I K 3 C A, A K T 1$} \\
\hline
\end{tabular}

AR, androgen receptor; BL1, basal-like 1; BL2, basal-like 2; BLIA, basal-like immune-activated; BLIS, basal-like immunosuppressed; EMT, epithelial-mesenchymal transition; ER; estrogen receptor; FISH, fluorescence in situ hybridization; HRD, homologous recombination deficiency; IHC, immunohistochemistry; IM, immunomodulatory; LAR, luminal androgen receptor; M, mesenchymal; MSL, mesenchymal stem-like; WGS, whole-genome sequencing.

(7.5 vs 5.0 months; HR, 0.62; $95 \% \mathrm{CI}$, 0.49-0.78; $P<.001) .{ }^{24}$ However, a more impressive and significant improvement in OS (25.4 vs 17.9 months; HR, 0.67; 95\% CI, 0.53-0.86) was demonstrated in the final analysis. ${ }^{25,26}$ This led to the FDA approval of atezolizumab in combination with nab-paclitaxel as first-line therapy for PD-L1-positive (defined as $\geq 1 \%$ in immune cells by the SP142 Ventana assay) mTNBC in 2019.

In contrast to the results of IMpassion130, the phase 3 IMpassion131 study (NCT03125902) evaluated atezolizumab in combination with paclitaxel compared with placebo plus paclitaxel and failed to demonstrate an improvement in PFS and OS. ${ }^{26}$ This study enrolled and randomized 651 participants in a 2:1 ratio to receive atezolizumab plus paclitaxel or placebo plus paclitaxel. The primary end point, PFS in the PD-L1-positive population, was not statistically different between the 2 arms (6.0 vs 5.7 months; HR, 0.82; $P=.20)$. Further research is needed to better understand these results, and how the difference of chemotherapy partner, and the impact of steroids in modulating a response to immunotherapy, may have contributed to the study's results. More research is also needed to understand how to optimize PD-L1 positivity evaluation to select patients who may benefit from immunotherapy. ${ }^{27}$

As per the FDA label, the atezolizumab approval is for "adult patients with unresectable locally advanced or metastatic TNBC whose tumors express PD-L1 (PD-L1 stained tumor-infiltrating immune cells of any intensity covering $\geq 1 \%$ of the tumor area), as determined by an FDA-approved test." This is particularly important because there is considerable variation in the median PFS estimates with immunotherapy depending on the type of assay used. ${ }^{27} \mathrm{~A}$ post hoc analysis evaluated PD-L1 status using 3 assays: Ventana SP142, SP263 immunohistochemistry (IHC) assay (positivity defined as $1 \%$ or more PD-L1 stained immune cells), or the Dako PD-L1 IHC 22C3 assay (positivity defined as 1 or more combined proportion score [CPS]). Patients in the SP142 PD-L1-positive population achieved the greatest clinical benefit from atezolizumab plus nab-paclitaxel. Thus, PD-L1 expression as a predictive biomarker needs to be assessed cautiously due to its variability with measurement assays and positivity thresholds both within and across tumor types. ${ }^{28}$

The optimal criteria and assay of PD-L1 positivity continued to be important considerations in KEYNOTE-355, the study of another immune checkpoint inhibitor, pembrolizumab (Keytruda). The phase 3 KEYNOTE-355 trial (NCT02819518) investigated pembrolizumab with chemotherapy (investigator's choice of either nab-paclitaxel, paclitaxel, or gemcitabine/ carboplatin) compared with placebo and chemotherapy for patients with previously untreated, advanced or metastatic TNBC whose tumors expressed PD-L1, defined by a CPS of 10 or greater, a different assay from the SP142/Ventana assay utilized in the IMpassion130 trial. ${ }^{29}$ The study enrolled 847 patients and demonstrated a statistically significant improvement in PFS with pembrolizumab and chemotherapy compared with chemotherapy alone $(9.7$ vs 5.6 months; HR, 0.65; $P=$ .0012 ) in the population with CPS 10 or greater. ${ }^{30}$ The study results led to the regulatory approval of pembrolizumab for patients with PD-L1-positive mTNBC, in combination with various chemotherapy partners, in November 2020. 
TABLE 2. Summary of Clinical Trials for Metastatic Triple-Negative Breast Cancer and the Efficacy Analysis by Biomarkers

\begin{tabular}{|c|c|c|c|c|c|c|c|c|}
\hline \multicolumn{9}{|c|}{ First-line therapy } \\
\hline Clinical trial & $\begin{array}{l}\text { Study } \\
\text { phase }\end{array}$ & Population & Treatment arms & Pts (N) & ORR (\%) & PFS (m) & OS (m) & Study ID (NCT) \\
\hline \multirow{4}{*}{ TNT } & \multirow{4}{*}{3} & \multirow{2}{*}{ Unselected first-line mTNBC } & Carboplatin & 188 & 31.4 & 3.1 & 12.8 & \multirow{4}{*}{$00532727^{61}$} \\
\hline & & & DTX & 188 & 34.0 & 4.4 & 12.0 & \\
\hline & & \multirow{2}{*}{ Germline $B R C A 1 / 2$ mut } & Carboplatin & 25 & 68.0 & 6.8 & NR & \\
\hline & & & DTX & 18 & 33.3 & 4.4 & NR & \\
\hline \multirow{2}{*}{ OlympiAD } & \multirow{2}{*}{3} & \multirow{2}{*}{$\begin{array}{l}\text { HER2-negative metastatic } \mathrm{BC} \\
\text { with germline } B R C A 1 / 2 \text { mut }\end{array}$} & Olaparib & 205 & 59.9 & 7.0 & 19.3 & \multirow{2}{*}{$02000622^{12}$} \\
\hline & & & Cape/eribulin, VNR & 97 & 28.8 & 4.2 & 19.6 & \\
\hline \multirow[b]{2}{*}{ EMBRACA } & \multirow[b]{2}{*}{3} & \multirow{2}{*}{$\begin{array}{l}\text { Advanced } \mathrm{BC} \text { with germline } \\
B R C A 1 / 2 \text { mut }\end{array}$} & Talazoparib & 287 & 61.8 & 5.8 & 22.3 & \multirow[b]{2}{*}{$01945775^{14,16,62}$} \\
\hline & & & $\begin{array}{l}\text { Eribulin/cape/VNR/ } \\
\text { GEM }\end{array}$ & 144 & 12.5 & 2.9 & 19.5 & \\
\hline \multirow{4}{*}{ IMpassion 130} & \multirow{4}{*}{3} & \multirow{2}{*}{ Unselected first-line mTNBC } & Atezo + nab-PTX & 451 & 56.0 & 7.2 & 21.0 & \multirow{4}{*}{$02425891^{24-26}$} \\
\hline & & & Nab-PTX & 451 & 45.9 & 5.5 & 18.7 & \\
\hline & & \multirow{2}{*}{ PD-L1 positive } & Atezo + nab-PTX & 185 & 58.9 & 7.5 & 25.4 & \\
\hline & & & Nab-PTX & 184 & 42.6 & 5.0 & 17.9 & \\
\hline \multirow{4}{*}{ IMpassion 131} & \multirow{4}{*}{3} & \multirow{2}{*}{ Unselected first-line mTNBC } & Atezo + PTX & 431 & 54.0 & 5.7 & 19.2 & \multirow{4}{*}{$03125902^{63}$} \\
\hline & & & PTX & 220 & 47.0 & 5.6 & 22.8 & \\
\hline & & \multirow[b]{2}{*}{ PD-L1 positive } & Atezo + PTX & 191 & 63.0 & 6.0 & 22.1 & \\
\hline & & & PTX & 101 & 55.0 & 5.7 & 28.3 & \\
\hline \multirow{4}{*}{ KEYNOTE-355 } & \multirow{4}{*}{3} & \multirow[t]{2}{*}{ mTNBC } & $\begin{array}{l}\text { Pembro + chemo- } \\
\text { therapy }\end{array}$ & 566 & \multirow[t]{2}{*}{ NR } & 7.5 & \multirow[t]{2}{*}{ NR } & \\
\hline & & & chemotherapy & 281 & & 5.6 & & 1001050 \\
\hline & & PD-L1 positive (CPS $\geq 10$ ) & $\begin{array}{l}\text { Pembro + chemo- } \\
\text { therapy }\end{array}$ & 220 & NR & 9.7 & NR & $02819518^{\circ 0}$ \\
\hline & & & chemotherapy & 103 & & 5.6 & & \\
\hline & & & Ipatasertib + PTX & 62 & 40.0 & 6.2 & 25.8 & \\
\hline & & mINBC & PTX & 62 & 32.0 & 4.9 & 16.9 & \\
\hline LOTUS & 2 & & Ipatasertib + PTX & 26 & 50.0 & 9.0 & 25.8 & $02162719^{33,34}$ \\
\hline & & PIKЗCA/AKT1/PTEN altered & PTX & 16 & 44.0 & 4.9 & 22.1 & \\
\hline & & mTNBC with PIK3CA/AKT1/ & Ipatasertib + PTX & 168 & 39.0 & 7.4 & NDP & 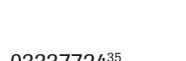 \\
\hline IPATunity130 & 3 & PTEN alteration & PTX & 87 & 35.0 & 6.1 & NR & $03337724^{35}$ \\
\hline & & TTRO & Capivasertib + PTX & 70 & 34.8 & 5.9 & 19.1 & \\
\hline & & & PTX & 70 & 28.8 & 4.2 & 12.6 & \\
\hline РАKI & 2 & & Capivasertib + PTX & 17 & 35.3 & 9.3 & NR & $02423603^{39}$ \\
\hline & & PIK3CA altered & PTX & 11 & 18.2 & 3.7 & 10.4 & \\
\hline
\end{tabular}


TABLE 2 CONTINUED. Summary of Clinical Trials for Metastatic Triple-Negative Breast Cancer and the Efficacy Analysis by Biomarkers

\begin{tabular}{|c|c|c|c|c|c|c|c|c|}
\hline Clinical trial & $\begin{array}{l}\text { Study } \\
\text { phase }\end{array}$ & Population & Treatment arms & Pts (N) & ORR (\%) & PFS (mo) & OS (m) & Study ID (NCT) \\
\hline \multirow{4}{*}{ KEYNOTE-119 } & \multirow{4}{*}{3} & \multirow[b]{2}{*}{ mTNBC } & Pembro & 312 & 9.6 & 2.1 & 9.9 & \multirow{4}{*}{$02555657^{64}$} \\
\hline & & & $\begin{array}{l}\text { Cape/eribulin/ } \\
\text { GEM/VNR }\end{array}$ & 310 & 10.6 & 3.3 & 10.8 & \\
\hline & & \multirow[b]{2}{*}{ PD-L1 positive $(C P S \geq 10)$} & Pembro & 96 & 17.7 & 2.1 & 12.7 & \\
\hline & & & $\begin{array}{l}\text { Cape/eribulin/ } \\
\text { GEM/VNR }\end{array}$ & 98 & 9.2 & 3.4 & 11.6 & \\
\hline Bardia et al & $1 / 2$ & mTNBC & $\begin{array}{l}\text { Sacituzumab } \\
\text { govitecan }\end{array}$ & 108 & 33.3 & 5.5 & 13.0 & $01631552^{45}$ \\
\hline \multirow{2}{*}{ ASCENT } & \multirow{2}{*}{3} & \multirow{2}{*}{ Pretreated mTNBC } & $\begin{array}{l}\text { Sacituzumab } \\
\text { govitecan }\end{array}$ & 235 & 35 & 5.6 & 12.1 & \multirow{2}{*}{$02574455^{46}$} \\
\hline & & & $\begin{array}{l}\text { Eribulin/cape/VNR/ } \\
\text { GEM }\end{array}$ & 233 & 5 & 1.7 & 6.7 & \\
\hline Modi et al & $1 b$ & HER2-low BC & $\begin{array}{l}\text { Trastuzumab } \\
\text { deruxtecan }\end{array}$ & 54 & 37.0 & 11.1 & NR & $02564900^{53}$ \\
\hline
\end{tabular}

atezo, atezolizumab; BC, breast cancer; cape, capecitabine, CPS, combined proportion score; DTX, docetaxel; GEM, gemcitabine; mTNBC, metastatic triple-negative breast cancer; mut, mutated; NR, not reported; ORR, objective response rate; OS, overall survival; pembro, pembrolizumab; PFS, progression-free survival; pts, patients; PTX, paclitaxel; VNR, vinorelbine.

\section{Targeting the PIK3CA/AKT/ PTEN Pathway}

The PIK3CA/AKT signaling pathway is often activated through activation mutations in PIK3CA or AKT1 or alterations in $P T E N^{31,32}$; also, approximately half of TNBC tumors have deficient expression of tumor suppressor PTEN, ${ }^{3}$ which is associated with AKT pathway activation. These findings have led to considerable therapeutic interest in evaluating AKT inhibitors, particularly in combination with chemotherapy as well as immunotherapy.

The LOTUS trial (NCT02162719), a randomized, double-blind, phase 2 trial, evaluated the efficacy of a selective oral ATP-competitive small molecule AKT inhibitor, ipatasertib, as first-line therapy in combination with paclitaxel, vs placebo and paclitaxel in patients with mTNBC. ${ }^{33}$ Between September 2014 and February 2016, 62 patients each were assigned to ipatasertib plus paclitaxel and to placebo plus paclitaxel. The addition of ipatasertib to paclitaxel was associated with a longer median PFS in the intention-to-treat population (6.2 vs 4.9 months; HR, 0.60; $95 \%$
CI, 0.37-0.98; $P=.037)$. Although the PFS in 48 patients with PTEN-low tumors assessed by IHC failed to demonstrate a statistically significant improvement with ipatasertib vs placebo, 42 patients with genetic PTEN-inactivating alterations or PIK3CA/AKT1-activating mutations demonstrated a PFS improvement with ipatasertib (9.0 vs 4.9 months; nonstratified HR, 0.44; 95\% CI, 0.20-0.99; $P=$ .041). ${ }^{33}$ The median OS was numerically longer in the ipatasertib arm compared with placebo (25.8 vs 16.9 months; HR, 0.80 ), but given the phase 2 design needed confirmation in a phase 3 trial $^{34}$.

As follow-up to the LOTUS trial, the IPATunity 130 phase 3 registration trial (NCT03337724) assigned 168 patients with advanced or metastatic TNBC with PIK3CA/AKT1/PTEN alterations to receive ipatasertib, and 87 patients to receive placebo, both combined with paclitaxel on days 1,8 , and 15 of 28day cycles. ${ }^{35}$ Surprisingly, there was no difference in median PFS between the 2 groups (7.4 vs 6.1 months; HR, 1.02; 95\% CI, 0.71-1.45; $P=.9237$ ) after
8.3 months of follow-up. Thus, the phase 3 trial failed to demonstrate the PFS benefit expected based on the results of the phase 2 LOTUS trial. The OS data are still immature, and a question that needs to be addressed is whether there may be a subpopulation among patients with PIK3CA/AKT1/PTEN-altered TNBC who may derive benefit from the drug. In addition, a pan-class I PI3K inhibitor, buparlisib, failed to improve the outcome. In the adaptive phase 2/3 BELLE-4 study (NCT01572727), 416 patients with HER2-negative locally advanced and metastatic breast cancer with no prior chemotherapy in the advanced setting were randomized 1:1 to receive either buparlisib plus paclitaxel or placebo plus paclitaxel. ${ }^{36}$ No PFS improvement occurred with buparlisib vs placebo in the entire cohort (8.9 vs 9.2 months, respectively; HR, 1.18) or PI3K pathway-activated cohort (9.1 vs 9.2 months, respectively; HR, 1.17). The results of this study also contrasted with the findings from the LOTUS trial. Capivasertib is another potent, highly selective oral small 
molecule that inhibits the isoforms of AKT1, AKT2, and AKT3. ${ }^{37,38}$ The PAKT trial (NCT02423603) is a double-blind, randomized phase 2 trial that enrolled patients with untreated mTNBC. Between May 2014 and June 2017, a total of 140 patients were randomly assigned to paclitaxel with either capivasertib or placebo in a 1:1 ratio. Similar to LOTUS, this study demonstrated an improvement in median PFS with capivasertib and paclitaxel compared with paclitaxel and placebo among 28 patients with genetic alterations of PI3CA, AKT1, PTEN (9.3 vs 3.7 months; HR, 0.30; 95\% CI, 0.11$0.79 ; P=.01) .{ }^{39}$ The most frequent serious AE was diarrhea, experienced in $13.2 \%$ of the capivasertib cohort.

Overall, the best approach to incorporate an $\mathrm{AKT}$ inhibitor into the treatment of TNBC has yet to be established. ${ }^{40}$ More specifically, questions remain as to which PTEN alterations confer sufficient loss of function to be targeted as frameshift mutations.

\section{Antibody-Drug Conjugates for $\mathrm{mTNBC}$}

Finally, ADCs have gained considerable momentum in the field of mTNBC. An ADC is composed of 3 well-defined components: a monoclonal antibody $(\mathrm{mAb})$, a cytotoxic payload, and a linker that connects them. A cancer-specific antibody binds to an antigen on the cell surface, gets internalized, and releases its potent payload to take effect. ${ }^{41}$ Apart from the selective delivery of ADC, the $\mathrm{mAb}$ induces apoptosis by inhibiting signal transduction ${ }^{42}$ or by the bystander killing effect, in which free payload in the cytosol permeates the plasma membrane in the tumor microenvironment to impact cells, including those that do not express the target antigen. ${ }^{43}$

Sacituzumab govitecan (IMMU-132; Trodelvy) is an ADC that targets Trop-2, which is overexpressed in the majority of TNBC, with the antibody linked with SN-38, a topoisomerase-1 inhibitor. ${ }^{44}$ In a basket single-arm phase $1 / 2$, multicenter trial enrolling patients with advanced epithelial cancers, sacituzumab govitecan demonstrated considerable single-agent activity among heavily pretreated patients with $\mathrm{mTNBC} .{ }^{45} \mathrm{~A}$ total of 108 patients received sacituzumab govitecan and the response rate was $33.3 \%$ (95\% CI, $24.6 \%-43.1 \%$ ) and the median PFS was 5.5 months $(95 \%$ CI, 4.1-6.3). This result compared favorably with the historical data of response rates with chemotherapy, thus leading to the accelerated approval of sacituzumab govitecan by the FDA in April 2020. Shortly after this approval, results of the confirmatory phase 3 trial (ASCENT; NCT02574455) investigating sacituzumab govitecan vs chemotherapy of physician's choice (eribulin, capecitabine, gemcitabine, or vinorelbine) in $\mathrm{mTNBC}$ were reported. This trial demonstrated a survival benefit from sacituzumab govitecan with a significantly improved median PFS (5.6 vs 1.7 months; HR, $0.41 ; P<.0001)$ and median OS (12.1 vs 6.7 months; HR, 0.48; $P<.0001)$ compared with chemotherapy of physician's choice. ${ }^{46}$ Also, sacituzumab govitecan induced clinical benefitimproved PFS and OS-over physician's choice of therapy in patients with mTNBC, irrespective of Trop-2 expression; however, greater efficacy was observed in those who had a medium or high Trop-2 score, according to data from an exploratory biomarker analysis of the phase 3 ASCENT trial that were presented during the 2020 San Antonio Breast Cancer Symposium. The most common any-grade AEs included nausea, neutropenia, diarrhea, fatigue, and anemia. Due to its compelling evidence of efficacy and no additional safety concerns, this trial was terminated early based on the recommendation by the independent data safety monitoring committee in March 2020. Collectively, these results demonstrate the promising potential of sacituzumab govitecan to impact the standard management of pretreated $\mathrm{mTNBC}$.
Similarly, ladiratuzumab vedotin, an ADC targeting LIV-1, combined with a microtubule inhibitor, monomethyl auristatin $\mathrm{E}$, as the toxic payload, has been developed for patients with relapsed/ refractory TNBC. ${ }^{47}$ As a monotherapy, ladiratuzumab vedotin is being studied to determine its safety and tolerability profile in patients with LIV-1-positive advanced or metastatic breast cancer, including 51 patients with $\mathrm{mTNBC}$, in a phase 1 , open-label, dose-escalation study (NCT01969643). Preliminary results from this trial have shown an objective response rate (ORR) of $32 \%$ and clinical benefit rate (CBR) of $36 \%$ with good tolerability, suggesting an encouraging antitumor signal in patients. ${ }^{48,49}$ In combination with immunotherapy, the synergistic effect of ladiratuzumab vedotin with pembrolizumab is being investigated in a single-arm, open-label, phase $1 \mathrm{~b} / 2$ study. ${ }^{50}$ The ongoing study including 26 patients with mTNBC showed an ORR of $54 \%$ with at least 3 months of follow-up.

In preclinical studies, the bystander killing effect mediated by anti-HER 2 ADCs showed the antitumor effect in HER2-low-expressing tumors, including HER2 IHC $1+$ or 2+, and HER3-positive tumors. ${ }^{51}$ Trastuzumab deruxtecan (DS-8201; Enhertu) is a humanized anti-HER $2 \mathrm{mAb}$ conjugated with a potent topoisomerase inhibitor, DX-8951 ${ }^{52}$; this drug demonstrated antitumor activity in HER2-low-expressing breast cancer, with an ORR of $37.0 \%$ and duration of response of 10.4 months in a phase $1 \mathrm{~b}$ trial. ${ }^{53}$ Trastuzumab deruxtecan was approved by the FDA for patients with HER2-positive metastatic breast cancer in December 2019, and its efficacy in HER2-low tumors is being assessed in an ongoing phase 3 clinical trial. ${ }^{54}$ Similarly, ongoing studies are evaluating combination of ADCs with targeted therapies, particularly therapeutic agents synergistic with the payload moiety..$^{55}$ The summary of various clinical trials and results by biomarkers is outlined in Table 2. 


\section{Other Targets}

A number of ongoing clinical trials are targeting key signaling pathways and molecular alterations in TNBC. For example, a subset of TNBC expresses the androgen receptor (AR), and there has been interest in targeting the receptor, similar to prostate cancer. ${ }^{56,57}$ In a pivotal phase 2 clinical trial, patients with AR-positive TNBC received bicalutamide. However, despite the biomarker selection, the efficacy was modest, with a 6-month CBR of $19 \% .{ }^{56}$ More encouraging results were seen with next-generation potent AR inhibitors such as enzalutamide, with a CBR (16 weeks) of $33 \%$ in patients with evaluable AR-positive TNBC. ${ }^{57}$ Further research is needed to develop better predictive biomarkers as well as combinatorial therapies for AR-positive TNBC. Another approach is to improve tolerability of chemotherapy by developing oral agents such as oral taxanes, as well as drugs like trilaciclib (Cosela) which can put bone marrow cells in G1 cell-cycle arrest, thereby reducing the myelosuppressive toxicity from chemotherapy and improving the efficacy/ toxicity ratio..$^{58,59}$

In summary, over the past few years there have been major strides in both understanding TNBC biology and in targeting TNBC with precision therapies. However, major questions and challenges remain. In particular, in the era of precision medicine, the need exists to develop biomarkers beyond routine genotyping. For example, can we identify patients with HRD-high subtype TNBC who could derive benefit from PARP inhibitors? Can we identify biomarkers to predict the response from single-agent immunotherapy? How can we identify activation of compensatory pathways and overcome resistance to targeted therapies? Successful clinical application of different therapies targeting different subsets of TNBC could potentially transform the therapeutic landscape of mTNBC and make the current definition of TNBC obsolete.

\section{FINANCIAL DISCLOSURE: The authors}

received no financial support for the research, authorship, and/or publication of this article.

AN owns stock of Chugai, Inc. Nagayama's immediate family member has a leadership position with Chugai, Inc, and Roche, Inc.

NV reports research funding to their institution (Massachusetts General Hospital) from Merck, Daehwa, Novartis, Pfizer (also travel reimbursement for conference presentation of ongoing study), and Radius, as well as prior advisory board participation with AbbVie.

$A B$ reports consultant/advisory board to Genentech/Roche, Immunomedics, Novartis, Pfizer, Merck, Radius Health, Daiichi Sankyo/ AstraZeneca, Sanofi, Puma Biotechnology, Phillips; and research funding to the institution: Genentech/Roche, Immunomedics, Novartis,

Pfizer, Merck, Radius Health, Sanofi, Mersana.

For full reference list, visit cancernetwork.com/Nagayama_5.21

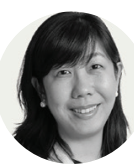

Nagayama

is from the Department of Surgery (Breast Group) at the Keio University School of Medicine in

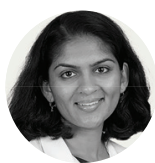

Boston, MA.

\section{Vidula}

is from the Massachusetts General Hospital Cancer Center and Harvard Medical School in

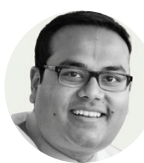

Boston, MA.

\section{Bardia}

is from the Massachusetts General Hospital Cancer Center and Harvard Medical School in

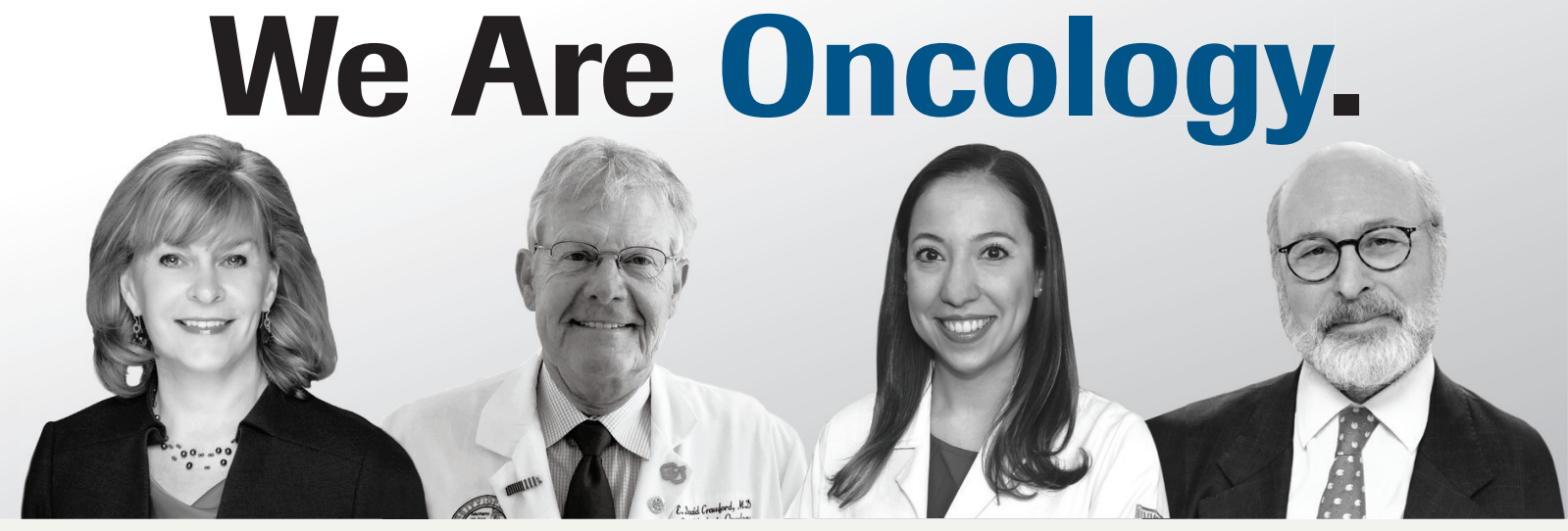

Peer review and perspective. More context-deeper insight. Explore us online. 


\section{For appropriate patients faced with relapsed/refractory multiple myeloma}

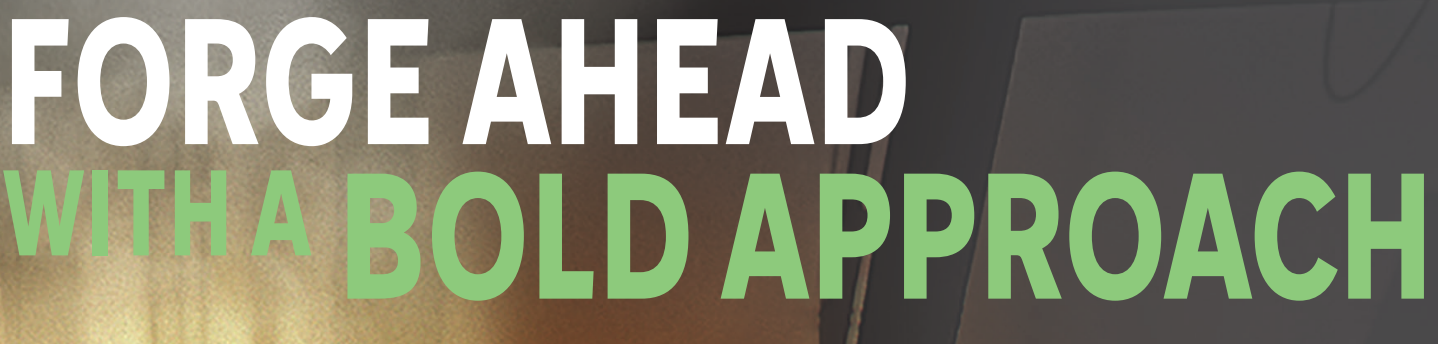

\section{Target BCMA for RRMM}

BLENREP is the first and only BCMA-targeted ADC monotherapy. So you can offer your RRMM patients a different option.

\section{INDICATION}

BLENREP is indicated for the treatment of adults with relapsed or refractory multiple myeloma who have received at least 4 prior therapies, including an anti-CD38 monoclonal antibody, a proteasome inhibitor, and an immunomodulatory agent.

This indication is approved under accelerated approval based on response rate. Continued approval for this indication may be contingent upon verification and description of clinical benefit in a confirmatory trial(s).

\section{IMPORTANT SAFETY INFORMATION}

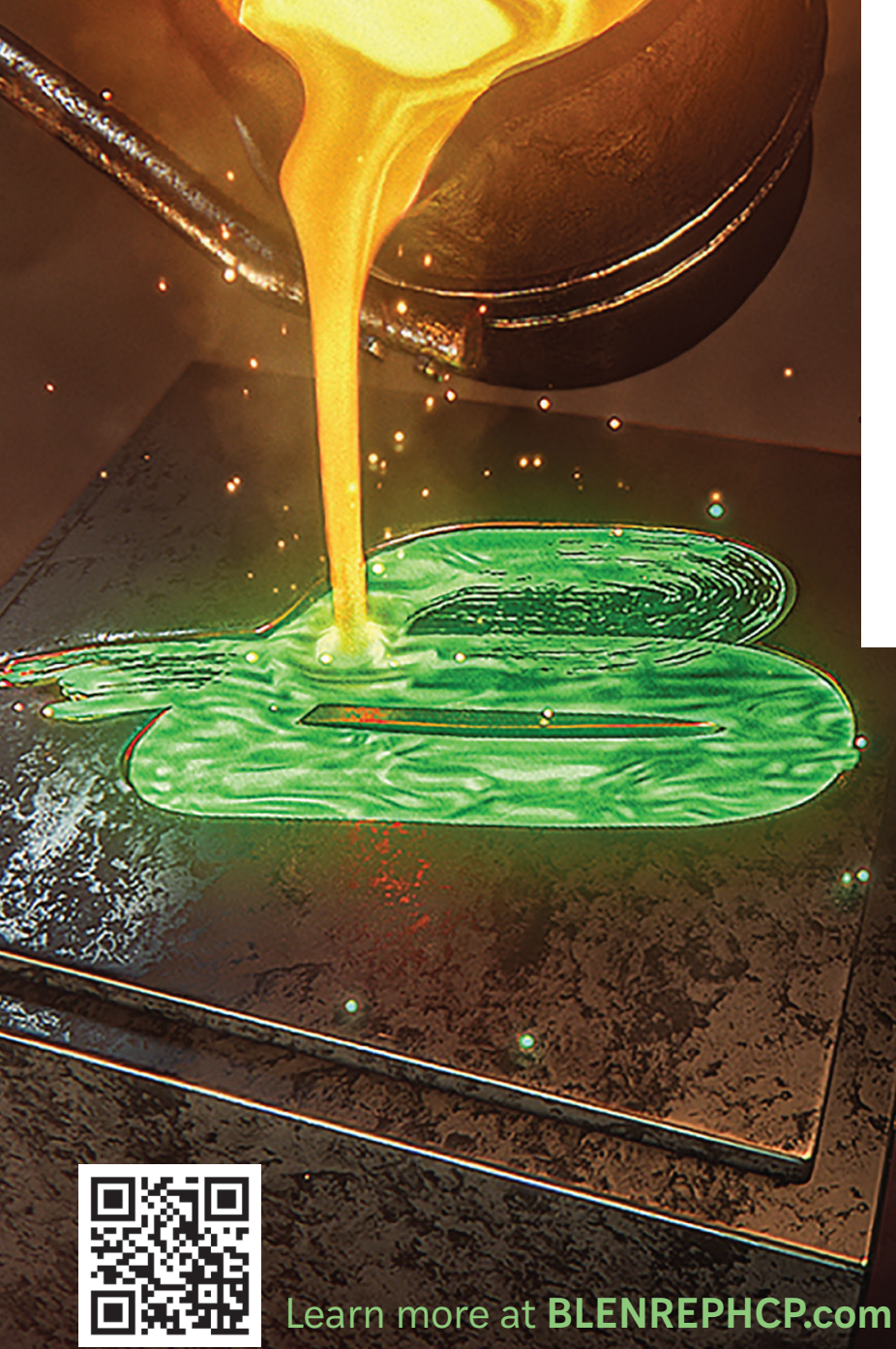




\section{IMPORTANT SAFETY INFORMATION} WARNINGS AND PRECAUTIONS

Ocular Toxicity: Ocular adverse reactions occurred in $77 \%$ of the 218 patients in the pooled safety population. Ocular adverse reactions included keratopathy (76\%), changes in visual acuity (55\%), blurred vision (27\%), and dry eye (19\%). Among patients with keratopathy ( $n=165)$, 49\% had ocular symptoms, $65 \%$ had clinically relevant visual acuity changes (decline of 2 or more lines on Snellen Visual Acuity in any eye), and $34 \%$ had both ocular symptoms and visual acuity changes.

Keratopathy: Keratopathy was reported as Grade 1 in $7 \%$ of patients, Grade 2 in 22\%, Grade 3 in 45\%, and Grade 4 in $0.5 \%$ per the KVA scale. Cases of corneal ulcer (ulcerative and infective keratitis) have been reported. Most keratopathy events developed within the first 2 treatment cycles (cumulative incidence of $65 \%$ by Cycle 2). Of the patients with Grade 2 to 4 keratopathy ( $n=149$ ), 39\% recovered to Grade 1 or lower after median follow-up of 6.2 months. Of the $61 \%$ who had ongoing keratopathy, $28 \%$ were still on treatment, $9 \%$ were in follow-up, and in $24 \%$ the follow-up ended due to death, study withdrawal, or lost to follow-up. For patients in whom events resolved, the median time to resolution was 2 months (range: 11 days to 8.3 months).

Visual Acuity Changes: A clinically significant decrease in visual acuity of worse than 20/40 in the better-seeing eye was observed in $19 \%$ of the 218 patients and of $20 / 200$ or worse in the better-seeing eye in $1.4 \%$. Of the patients with decreased visual acuity of worse than 20/40, 88\% resolved and the median time to resolution was 22 days (range: 7 days to 4.2 months). Of the patients with decreased visual acuity of 20/200 or worse, all resolved and the median duration was 22 days (range: 15 to 22 days).

Monitoring and Patient Instruction: Conduct ophthalmic examinations (visual acuity and slit lamp) at baseline, prior to each dose, and promptly for worsening symptoms. Perform baseline examinations within 3 weeks prior to the first dose. Perform each follow-up examination at least 1 week after the previous dose and within 2 weeks prior to the next dose. Withhold BLENREP until improvement and resume at same or reduced dose, or consider permanently discontinuing based on severity. Advise patients to use preservative-free lubricant eye drops at least 4 times a day starting with the first infusion and continuing until end of treatment. Avoid use of contact lenses unless directed by an ophthalmologist. Changes in visual acuity may be associated with difficulty for driving and reading. Advise patients to use caution when driving or operating machinery. BLENREP is only available through a restricted program under a REMS.

Thrombocytopenia: Thrombocytopenia occurred in 69\% of 218 patients in the pooled safety population, including Grade 2 in 13\%, Grade 3 in $10 \%$, and Grade 4 in 17\%. The median time to onset of the first thrombocytopenic event was 26.5 days.

Thrombocytopenia resulted in dose reduction, dose interruption, or discontinuation in $9 \%, 2.8 \%$, and $0.5 \%$ of patients, respectively. Grade 3 to 4 bleeding events occurred in $6 \%$ of patients, including Grade 4 in 1 patient. Fatal adverse reactions included cerebral hemorrhage in 2 patients. Perform complete blood cell counts at baseline and during treatment as clinically indicated. Consider withholding and/or reducing the dose based on severity.

Infusion-Related Reactions: Infusion-related reactions occurred in $18 \%$ of 218 patients in the pooled safety population, including Grade 3 in 1.8\%. Monitor patients for infusion-related reactions. For Grade 2 or 3 reactions, interrupt the infusion and provide supportive treatment. Once symptoms resolve, resume at a lower infusion rate. Administer premedication for all subsequent infusions. Discontinue BLENREP for life-threatening infusion-related reactions and provide appropriate emergency care.

Embryo-Fetal Toxicity: Based on its mechanism of action, BLENREP can cause fetal harm when administered to a pregnant woman. Advise pregnant women of the potential risk to a fetus.
Advise females of reproductive potential to use effective contraception during treatment with BLENREP and for 4 months after the last dose. Advise males with female partners of reproductive potential to use effective contraception during treatment with BLENREP and for 6 months after the last dose. Pregnancy testing is recommended for females of reproductive potential prior to initiating BLENREP.

\section{ADVERSE REACTIONS}

The pooled safety population described in Warnings and Precautions reflects exposure to BLENREP at a dosage of $2.5 \mathrm{mg} / \mathrm{kg}$ or $3.4 \mathrm{mg} / \mathrm{kg}$ (1.4 times the recommended dose) administered intravenously once every 3 weeks in 218 patients in DREAMM-2. Of these patients, 194 received a liquid formulation (not the approved dosage form) rather than the lyophilized powder.

Patients received BLENREP at the recommended dosage of $2.5 \mathrm{mg} / \mathrm{kg}$ administered intravenously once every 3 weeks $(n=95)$. Permanent discontinuation due to an adverse reaction occurred in $8 \%$ of patients who received BLENREP; keratopathy (2.1\%) was the most frequent adverse reaction resulting in permanent discontinuation. Dosage interruptions due to an adverse reaction occurred in $54 \%$ of patients who received BLENREP. Adverse reactions which required a dosage interruption in $>3 \%$ of patients included keratopathy (47\%), blurred vision (5\%), dry eye (3.2\%), and pneumonia (3.2\%). Dose reductions due to an adverse reaction occurred in $29 \%$ of patients. Adverse reactions which required a dose reduction in $>3 \%$ of patients included keratopathy (23\%) and thrombocytopenia (5\%).

The most common adverse reactions ( $\geq 20 \%$ ) were keratopathy (71\%), decreased visual acuity (53\%), nausea (24\%), blurred vision (22\%), pyrexia (22\%), infusion-related reactions (21\%), and fatigue $(20 \%)$. The most common Grade 3 or $4(\geq 5 \%)$ laboratory abnormalities were lymphocytes decreased $(22 \%)$, platelets decreased (21\%), hemoglobin decreased (18\%), neutrophils decreased (9\%), creatinine increased (5\%), and gamma-glutamyl transferase increased (5\%).

Serious adverse reactions occurred in $40 \%$ of patients who received BLENREP. Serious adverse reactions in $>3 \%$ of patients included pneumonia (7\%), pyrexia (6\%), renal impairment (4.2\%), sepsis (4.2\%), hypercalcemia (4.2\%), and infusion-related reactions (3.2\%). Fatal adverse reactions occurred in $3.2 \%$ of patients, including sepsis (1\%), cardiac arrest (1\%), and lung infection (1\%).

\section{USE IN SPECIFIC POPULATIONS}

Lactation: Because of the potential for serious adverse reactions in the breastfed child, advise women not to breastfeed during treatment with BLENREP and for 3 months after the last dose.

Females and Males of Reproductive Potential: Based on findings in animal studies, BLENREP may impair fertility in females and males.

Geriatric Use: Of the 218 patients who received BLENREP in DREAMM-2, 43\% were aged 65 to less than 75 years and $17 \%$ were aged 75 years and older. Keratopathy occurred in $80 \%$ of patients aged less than 65 years and $73 \%$ of patients aged 65 years and older. Among the 95 patients who received BLENREP at the $2.5-\mathrm{mg} / \mathrm{kg}$ dose, keratopathy occurred in $67 \%$ of patients aged less than 65 years and $73 \%$ of patients aged 65 years and older.

Renal or Hepatic Impairment: The recommended dosage has not been established in patients with severe renal impairment (eGFR 15 to $29 \mathrm{~mL} / \mathrm{min} / 1.73 \mathrm{~m}^{2}$ ) or end-stage renal disease (ESRD) with eGFR $<15 \mathrm{~mL} / \mathrm{min} / 1.73 \mathrm{~m}^{2}$ not on dialysis or requiring dialysis. The recommended dosage has not been established in patients with moderate or severe hepatic impairment (total bilirubin $>1.5 \times U L N$ and any AST).

Please see Brief Summary of full Prescribing Information, including BOXED WARNING, on the following pages. 


\section{BLENREP}

\section{(belantamab mafodotin-blmf)}

for injection, for intravenous use

The following is a brief summary only; see full Prescribing Information for complete product information.

WARNING: OCULAR TOXICITY
BLENREP caused changes in the corneal epithelium resulting in
changes in vision, including severe vision loss and corneal ulcer,
and symptoms, such as blurred vision and dry eyes [see Warnings
and Precautions (5.1)].
Conduct ophthalmic exams at baseline, prior to each dose, and
promptly for worsening symptoms. Withhold BLENREP until
improvement and resume, or permanently discontinue, based on
severity [see Dosage and Administration (2.3) of full Prescribing
Information, Warnings and Precautions (5.1)].
Because of the risk of ocular toxicity, BLENREP is available
only through a restricted program under a Risk Evaluation
and Mitigation Strategy (REMS) called the BLENREP REMS
[see Warnings and Precautions (5.2)].

\section{INDICATIONS AND USAGE}

BLENREP is indicated for the treatment of adults with relapsed or refractory multiple myeloma who have received at least 4 prior therapies, including an anti-CD38 monoclonal antibody, a proteasome inhibitor, and an immunomodulatory agent. This indication is approved under accelerated approval based on response rate [see Clinical Studies (14) of full Prescribing Information]. Continued approval for this indication may be contingent upon verification and description of clinical benefit in a confirmatory trial(s).

\section{CONTRAINDICATIONS}

None.

\section{WARNINGS AND PRECAUTIONS}

\subsection{Ocular Toxicity}

0cular adverse reactions occurred in $77 \%$ of the 218 patients in the pooled safety population. Ocular adverse reactions included keratopathy $(76 \%)$, changes in visual acuity (55\%), blurred vision (27\%), and dry eye (19\%) [see Adverse Reactions (6.1)] Among patients with keratopathy ( $n=165), 49 \%$ had ocular symptoms, $65 \%$ had clinically relevant visual acuity changes (decline of 2 or more lines on Snellen Visual Acuity in any eye), and $34 \%$ had both ocular symptoms and visual acuity changes.

\section{Keratopathy}

Keratopathy was reported as Grade 1 in $7 \%$ of patients, Grade 2 in $22 \%$, Grade 3 in $45 \%$, and Grade 4 in $0.5 \%$ per the KVA scale. Cases of corneal ulcer (ulcerative and infective keratitis) have been reported. Most keratopathy events developed within the first 2 treatment cycles (cumulative incidence of $65 \%$ by Cycle 2). Of the patients with Grade 2 to 4 keratopathy $(n=149), 39 \%$ of patients recovered to Grade 1 or lower after median follow-up of 6.2 months. Of the $61 \%$ who had ongoing keratopathy, $28 \%$ were still on treatment, $9 \%$ were in follow-up, and in $24 \%$ the follow-up ended due to death, study withdrawal, or lost to follow up. For patients in whom events resolved, the median time to resolution was 2 months (range: 11 days to 8.3 months).

\section{Visual Acuity Changes}

A clinically significant decrease in visual acuity of worse than 20/40 in the better-seeing eye was observed in $19 \%$ of the 218 patients and of $20 / 200$ or worse in the better-seeing eye in $1.4 \%$. Of the patients with decreased visual acuity of worse than $20 / 40,88 \%$ resolved and the median time to resolution was 22 days (range: 7 days to 4.2 months). Of the patients with decreased visual acuity of $20 / 200$ or worse, all resolved and the median duration was 22 days (range: 15 to 22 days).

\section{Monitoring and Patient Instruction}

Conduct ophthalmic examinations (visual acuity and slit lamp) at baseline, prior to each dose, and promptly for worsening symptoms. Perform baseline examinations within 3 weeks prior to the first dose. Perform each follow-up examination at least 1 week after the previous dose and within 2 weeks prior to the next dose. Withhold BLENREP until improvement and resume at same or reduced dose, or consider permanently discontinuing based on severity [see Dosage and Administration (2.3) of full Prescribing Information].

Advise patients to use preservative-free lubricant eye drops at least 4 times a day starting with the first infusion and continuing until end of treatment. Avoid use of contact lenses unless directed by an ophthalmologist [see Dosage and Administration (2.1) of full Prescribing Information].

Changes in visual acuity may be associated with difficulty for driving and reading. Advise patients to use caution when driving or operating machinery.

BLENREP is only available through a restricted program under a REMS [see Warnings and Precautions (5.2)].

\subsection{BLENREP REMS}

BLENREP is available only through a restricted program under a REMS called the BLENREP REMS because of the risks of ocular toxicity [see Warnings and Precautions (5.1)].

Notable requirements of the BLENREP REMS include the following:

- Prescribers must be certified with the program by enrolling and completing training in the BLENREP REMS.

- Prescribers must counsel patients receiving BLENREP about the risk of ocular toxicity and the need for ophthalmic examinations prior to each dose.

- Patients must be enrolled in the BLENREP REMS and comply with monitoring.

- Healthcare facilities must be certified with the program and verify that patients are authorized to receive BLENREP.

-Wholesalers and distributers must only distribute BLENREP to certified healthcare facilities.

Further information is available, at www.BLENREPREMS.com and 1-855-209-9188.

\subsection{Thrombocytopenia}

Thrombocytopenia occurred in $69 \%$ of 218 patients in the pooled safety population, including Grade 2 in 13\%, Grade 3 in 10\%, and Grade 4 in 17\% [see Adverse Reactions (6.1)]. The median time to onset of the first thrombocytopenic event was 26.5 days. Thrombocytopenia resulted in dose reduction, dose interruption, or discontinuation in $9 \%, 2.8 \%$, and $0.5 \%$ of patients, respectively.

Grade 3 to 4 bleeding events occurred in 6\% of patients, including Grade 4 in 1 patient. Fatal adverse reactions included cerebral hemorrhage in 2 patients.

Perform complete blood cell counts at baseline and during treatment as clinically indicated. Consider withholding and/or reducing the dose based on severity [see Dosage and Administration (2.3) of full Prescribing Information].

\subsection{Infusion-Related Reactions}

Infusion-related reactions occurred in $18 \%$ of 218 patients in the pooled safety population, including Grade 3 in 1.8\% [see Adverse Reactions (6.1)].

Monitor patients for infusion-related reactions. For Grade 2 or 3 reactions, interrupt the infusion and provide supportive treatment. Once symptoms resolve, resume at a lower infusion rate [see Dosage and Administration (2.3) of full Prescribing Information]. Administer premedication for all subsequent infusions. Discontinue BLENREP for life-threatening infusion-related reactions and provide appropriate emergency care.

\subsection{Embryo-Fetal Toxicity}

Based on its mechanism of action, BLENREP can cause fetal harm when administered to a pregnant woman because it contains a genotoxic compound (the microtubule inhibitor, monomethyl auristatin F [MMAF]) and it targets actively dividing cells.

Advise pregnant women of the potential risk to a fetus. Advise females of reproductive potential to use effective contraception during treatment with BLENREP and for 4 months after the last dose. Advise males with female partners of reproductive potential to use effective contraception during treatment with BLENREP and for 6 months after the last dose [see Use in Specific Populations $(8.1,8.3)]$.

\section{ADVERSE REACTIONS}

The following clinically significant adverse reactions are described elsewhere in the labeling:

- Ocular toxicity [see Warnings and Precautions (5.1)].

- Thrombocytopenia [see Warnings and Precautions (5.3)].

- Infusion-related reactions [see Warnings and Precautions (5.4)].

\subsection{Clinical Trials Experience}

Because clinical trials are conducted under widely varying conditions, adverse reaction rates observed in the clinical trials of a drug cannot be directly compared with rates in the clinical trials of another drug and may not reflect the rates observed in practice.

The pooled safety population described in Warnings and Precautions reflects exposure to BLENREP at a dosage of $2.5 \mathrm{mg} / \mathrm{kg}$ or $3.4 \mathrm{mg} / \mathrm{kg}$ (1.4 times the recommended dose) administered intravenously once every 3 weeks in 218 patients in DREAMM-2. Of these patients, 194 received a liquid formulation (not the approved dosage form) rather than the lyophilized powder. Among the 218 patients, $24 \%$ were exposed for 6 months or longer.

Relapsed or Refractory Multiple Myeloma

The safety of BLENREP as a single agent was evaluated in DREAMM-2 [see Clinical Studies (14.1) of full Prescribing Information]. Patients received BLENREP at the recommended dosage of $2.5 \mathrm{mg} / \mathrm{kg}$ administered intravenously once every 3 weeks ( $n=95$ ). Among these patients, $22 \%$ were exposed for 6 months or longer.

Serious adverse reactions occurred in $40 \%$ of patients who received BLENREP Serious adverse reactions in $>3 \%$ of patients included pneumonia $(7 \%)$, pyrexia $(6 \%)$, renal impairment (4.2\%), sepsis (4.2\%), hypercalcemia (4.2\%), and infusion-related reactions (3.2\%). Fatal adverse reactions occurred in $3.2 \%$ of patients, including sepsis (1\%), cardiac arrest (1\%), and lung infection (1\%). 
Permanent discontinuation due to an adverse reaction occurred in $8 \%$ of patients who received BLENREP; keratopathy $(2.1 \%)$ was the most frequent adverse reaction resulting in permanent discontinuation.

Dosage interruptions due to an adverse reaction occurred in $54 \%$ of patients who received BLENREP. Adverse reactions which required a dosage interruption in $>3 \%$ of patients included keratopathy $(47 \%)$, blurred vision (5\%), dry eye $(3.2 \%)$, and pneumonia (3.2\%).

Dose reductions due to an adverse reaction occurred in $29 \%$ of patients. Adverse reactions which required a dose reduction in $>3 \%$ of patients included keratopathy (23\%) and thrombocytopenia (5\%)

The most common adverse reactions ( $\geq 20 \%$ ) were keratopathy, decreased visua acuity, nausea, blurred vision, pyrexia, infusion-related reactions, and fatigue. The most common Grade 3 or $4(\geq 5 \%)$ laboratory abnormalities were lymphocytes decreased, platelets decreased, hemoglobin decreased, neutrophils decreased, creatinine increased, and gamma-glutamyl transferase increased.

Table 1 summarizes the adverse reactions in DREAMM-2 for patients who received the recommended dosage of $2.5 \mathrm{mg} / \mathrm{kg}$ once every 3 weeks.

Table 1. Adverse Reactions ( $\geq 10 \%$ ) in Patients Who Received BLENREP in DREAMM-2

\begin{tabular}{|c|c|c|}
\hline \multirow[b]{2}{*}{ Adverse Reactions } & \multicolumn{2}{|c|}{$\begin{array}{c}\text { BLENREP } \\
\mathbf{N}=95\end{array}$} \\
\hline & $\begin{array}{c}\text { All Grades } \\
(\%)\end{array}$ & $\begin{array}{c}\text { Grade 3-4 } \\
\text { (\%) }\end{array}$ \\
\hline \multicolumn{3}{|l|}{ Eye disorders } \\
\hline Keratopathy ${ }^{\mathrm{a}}$ & 71 & 44 \\
\hline Decreased visual acuity ${ }^{b}$ & 53 & 28 \\
\hline Blurred vision ${ }^{c}$ & 22 & 4 \\
\hline Dry eyes $^{d}$ & 14 & 1 \\
\hline \multicolumn{3}{|l|}{ Gastrointestinal disorders } \\
\hline Nausea & 24 & 0 \\
\hline Constipation & 13 & 0 \\
\hline Diarrhea & 13 & 1 \\
\hline \multicolumn{3}{|c|}{ General disorders and administration site conditions } \\
\hline Pyrexia & 22 & 3 \\
\hline Fatigue $^{\mathrm{e}}$ & 20 & 2 \\
\hline \multicolumn{3}{|l|}{ Procedural complications } \\
\hline Infusion-related reactions $^{f}$ & 21 & 3 \\
\hline \multicolumn{3}{|c|}{ Musculoskeletal and connective tissue disorders } \\
\hline Arthralgia & 12 & 0 \\
\hline Back pain & 11 & 2 \\
\hline \multicolumn{3}{|c|}{ Metabolic and nutritional disorders } \\
\hline Decreased appetite & 12 & 0 \\
\hline \multicolumn{3}{|l|}{ Infections } \\
\hline Upper respiratory tract infection ${ }^{g}$ & 11 & 0 \\
\hline
\end{tabular}

a Keratopathy was based on slit lamp eye examination, characterized as corneal epithelium changes with or without symptoms.

${ }^{b}$ Visual acuity changes were determined upon eye examination.

'Blurred vision included diplopia, vision blurred, visual acuity reduced, and visual impairment.

${ }^{\mathrm{d}}$ Dry eyes included dry eye, ocular discomfort, and eye pruritus.

${ }^{\text {e}}$ Fatigue included fatigue and asthenia.

f Infusion-related reactions included infusion-related reaction, pyrexia, chills, diarrhea, nausea, asthenia, hypertension, lethargy, tachycardia.

g Upper respiratory tract infection included upper respiratory tract infection, nasopharyngitis, rhinovirus infections, and sinusitis.

Clinically relevant adverse reactions in $<10 \%$ of patients included: Eye Disorders: Photophobia, eye irritation, infective keratitis, ulcerative keratitis. Gastrointestinal Disorders: Vomiting

Infections: Pneumonia.

Investigations: Albuminuria.
Table 2 summarizes the laboratory abnormalities in DREAMM-2.

Table 2. Laboratory Abnormalities ( $\geq 20 \%$ ) Worsening from Baseline in Patients Who Received BLENREP in DREAMM-2

\begin{tabular}{|l|c|c|}
\hline \multirow{2}{*}{\multicolumn{2}{c}{ Laboratory Abnormality }} & \multicolumn{2}{c|}{$\begin{array}{c}\text { BLENREP } \\
\text { N=95 }\end{array}$} \\
\cline { 2 - 3 } & $\begin{array}{c}\text { All Grades } \\
\text { (\%) }\end{array}$ & $\begin{array}{c}\text { Grades 3-4 } \\
\text { (\%) }\end{array}$ \\
\hline Hematology & 62 & 21 \\
\hline Platelets decreased & 49 & 22 \\
\hline Lymphocytes decreased & 32 & 18 \\
\hline Hemoglobin decreased & 28 & 9 \\
\hline Neutrophils decreased & 57 & 2 \\
\hline Chemistry & 43 & 4 \\
\hline Aspartate aminotransferase increased & 38 & 3 \\
\hline Albumin decreased & 28 & 5 \\
\hline Glucose increased & 26 & 1 \\
\hline Creatinine increased & 25 & 5 \\
\hline Alkaline phosphatase increased & 22 & 1 \\
\hline Gamma-glutamyl transferase increased & 21 & 2 \\
\hline Creatinine phosphokinase increased & 20 & 2 \\
\hline Sodium decreased & & \\
\hline Potassium decreased & \multicolumn{2}{|c|}{} \\
\hline
\end{tabular}

\subsection{Immunogenicity}

As with all therapeutic proteins, there is potential for immunogenicity. The detection of antibody formation is highly dependent on the sensitivity and specificity of the assay. Additionally, the observed incidence of antibody (including neutralizing antibody) positivity in an assay may be influenced by several factors including assay methodology, sample handling, timing of sample collection, concomitant medications, and underlying disease. For these reasons, comparison of the incidence of antibodies in the studies described below with the incidence of antibodies in other studies or to other products may be misleading.

The immunogenicity of BLENREP was evaluated using an electrochemiluminescence (ECL)-based immunoassay to test for anti-belantamab mafodotin antibodies. In clinical studies of BLENREP, $2 / 274$ patients $(<1 \%)$ tested positive for antibelantamab mafodotin antibodies after treatment. One of the 2 patients tested positive for neutralizing anti-belantamab mafodotin antibodies following 4 weeks on therapy. Due to the limited number of patients with antibodies against belantamab mafodotinblmf, no conclusions can be drawn concerning a potential effect of immunogenicity on pharmacokinetics, efficacy, or safety.

\section{USE IN SPECIFIC POPULATIONS}

\subsection{Pregnancy}

\section{Risk Summary}

Based on its mechanism of action, BLENREP can cause fetal harm when administered to a pregnant woman, because it contains a genotoxic compound (the microtubule inhibitor, MMAF) and it targets actively dividing cells [see Clinical Pharmacology (12.1), Nonclinical Toxicology (13.1) of full Prescribing Information]. Human immunoglobulin $\mathrm{G}(\mathrm{lgG})$ is known to cross the placenta; therefore, belantamab mafodotin-blmf has the potential to be transmitted from the mother to the developing fetus. There are no available data on the use of BLENREP in pregnant women to evaluate for drugassociated risk. No animal reproduction studies were conducted with BLENREP. Advise pregnant women of the potential risk to a fetus.

The estimated background risk of major birth defects and miscarriage for the indicated population is unknown. All pregnancies have a background risk of birth defect, loss, or other adverse outcome. In the U.S. general population, the estimated background risk of major birth defects and miscarriage in clinically recognized pregnancies is $2 \%$ to $4 \%$ and $15 \%$ to $20 \%$, respectively.

Data

Animal Data: Animal reproductive or developmental toxicity studies were not conducted with belantamab mafodotin-blmf. The cytotoxic component of BLENREP, MMAF, disrupts microtubule function, is genotoxic, and can be toxic to rapidly dividing cells, suggesting it has the potential to cause embryotoxicity and teratogenicity. 


\subsection{Lactation}

\section{Risk Summary}

There is no data on the presence of belantamab mafodotin-blmf in human milk or the effects on the breastfed child or milk production. Because of the potential for serious adverse reactions in the breastfed child, advise women not to breastfeed during treatment with BLENREP and for 3 months after the last dose.

\subsection{Females and Males of Reproductive Potential}

BLENREP can cause fetal harm when administered to pregnant women [see Use in Specific Populations (8.1)]

\section{Pregnancy Testing}

Pregnancy testing is recommended for females of reproductive potential prior to initiating BLENREP.

\section{Contraception}

Females: Advise women of reproductive potential to use effective contraception during treatment and for 4 months after the last dose.

Males: Because of the potential for genotoxicity, advise males with female partners of reproductive potential to use effective contraception during treatment with BLENREP and for 6 months after the last dose [see Nonclinical Toxicology (13.1) of full Prescribing Information].

Infertility

Based on findings in animal studies, BLENREP may impair fertility in females and males. The effects were not reversible in male rats, but were reversible in female rats [see Nonclinical Toxicology (13.1) of full Prescribing Information].

\subsection{Pediatric Use}

The safety and effectiveness of BLENREP in pediatric patients have not been established.

\subsection{Geriatric Use}

Of the 218 patients who received BLENREP in DREAMM-2, 43\% were aged 65 to less than 75 years and $17 \%$ were aged 75 years and older. Clinical studies of BLENREP did not include sufficient numbers of patients aged 65 and older to determine whether the effectiveness differs compared with that of younger patients. Keratopathy occurred in $80 \%$ of patients aged less than 65 years and $73 \%$ of patients aged 65 years and older. Among the patients who received BLENREP at the $2.5-\mathrm{mg} / \mathrm{kg}$ dose in DREAMM-2 $(n=95)$, keratopathy occurred in $67 \%$ of patients aged less than 65 years and $73 \%$ of patients aged 65 years and older. Clinical studies did not include sufficient numbers of patients 75 years and older to determine whether they respond differently compared with younger patients.

\subsection{Renal Impairment}

No dose adjustment is recommended for patients with mild or moderate renal impairment (estimated glomerular filtration rate [eGFR] 30 to $89 \mathrm{~mL} / \mathrm{min} / 1.73 \mathrm{~m}^{2}$ as estimated by the Modification of Diet in Renal Disease [MDRD] equation) [see Clinical Pharmacology (12.3) of full Prescribing Information]. The recommended dosage has not been established in patients with severe renal impairment (eGFR 15 to $29 \mathrm{~mL} / \mathrm{min} / 1.73 \mathrm{~m}^{2}$ ) or end-stage renal disease (ESRD) with $\mathrm{eGFR}<15 \mathrm{~mL} / \mathrm{min} / 1.73 \mathrm{~m}^{2}$ not on dialysis or requiring dialysis [see Clinical Pharmacology (12.3) of full Prescribing Information].

\subsection{Hepatic Impairment}

No dose adjustment is recommended for patients with mild hepatic impairment (total bilirubin $\leq$ upper limit of normal [ULN] and aspartate aminotransferase (AST) $>$ ULN or total bilirubin 1 to $\leq 1.5 \times$ ULN and any AST).

The recommended dosage of BLENREP has not been established in patients with moderate or severe hepatic impairment (total bilirubin $>1.5 \times$ ULN and any AST) [see Clinical Pharmacology (12.3) of full Prescribing Information].

\section{PATIENT COUNSELING INFORMATION}

Advise the patient to read the FDA-approved patient labeling (Medication Guide).

\section{Ocular Toxicity}

- Advise patients that ocular toxicity may occur during treatment with BLENREP [see Warnings and Precautions (5.1)].

- Advise patients to administer preservative-free lubricant eye drops as recommended during treatment and to avoid wearing contact lenses during treatment unless directed by a healthcare professional [see Dosage and Administration (2.3) of full Prescribing Information, Warnings and Precautions (5.1)].

- Advise patients to use caution when driving or operating machinery as BLENREP may adversely affect their vision [see Warnings and Precautions (5.1)].

\section{BLENREP REMS}

BLENREP is available only through a restricted program called BLENREP REMS [see Warnings and Precautions (5.2)]. Inform the patient of the following notable requirements:

- Patients must complete the enrollment form with their provider.

- Patients must comply with ongoing monitoring for eye exams [see Warnings and Precautions (5.1)].

Thrombocytopenia

- Advise patients to inform their healthcare provider if they develop signs or symptoms of bleeding [see Warnings and Precautions (5.3)].

Infusion-Related Reactions

- Advise patients to immediately report any signs and symptoms of infusion-related reactions to their healthcare provider [see Warnings and Precautions (5.4)].

Embryo-Fetal Toxicity

- Advise pregnant women of the potential risk to a fetus. Advise females of reproductive potential to inform their healthcare provider of a known or suspected pregnancy [see Warnings and Precautions (5.5), Use in Specific Populations (8.1, 8.3)].

- Advise women of reproductive potential to use highly effective contraception during treatment and for 4 months after the last dose [see Warnings and Precautions (5.5), Use in Specific Populations (8.3)].

- Advise males with female partners of reproductive potential to use effective contraception during treatment with BLENREP and for 6 months after the last dose [see Use in Specific Populations (8.3), Nonclinical Toxicology (13.1) of full Prescribing Information].

Lactation

- Advise women not to breastfeed during treatment with BLENREP and

for 3 months after the last dose [see Use in Specific Populations (8.2)].

Infertility

- Advise males and females of reproductive potential that BLENREP may impair fertility [see Use in Specific Populations (8.3)].

Trademarks are owned by or licensed to the GSK group of companies.

Manufactured by:

GlaxoSmithKline Intellectual Property Development Ltd. England

Brentford, Middlesex, UK TW8 9GS

U.S. License No. 2148

including by use of Potelligent technology licensed from BioWa, Inc.

For:

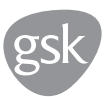

GlaxoSmithKline

Research Triangle Park, NC 27709

(C2020 GSK group of companies or its licensor.

August 2020 BRP:1BRS

(C)2021 GSK or licensor.

BLMADVT190001 January 2021

Produced in USA.
BLENREP belantamab mafodotin-blmf for injection $100 \mathrm{mg}$ 


\section{Primary Focal Therapy for Localized Prostate Cancer: A Review of the Literature}

Mitch Hayes, MD; Michael Lin-Brande, MD; and Sudhir Isharwal, MD

\begin{abstract}
:
BACKGROUND: Currently established treatment strategies for localized prostate cancer (PCa) include active surveillance and whole-gland treatments, such as radical prostatectomy and radiation therapy. These whole-gland treatments are not uncommonly associated with significant morbidity. Recent studies suggest that a single index lesion drives the disease progression in most cases. With improvements in pretreatment imaging modalities such as multiparametric MRI, the prostate's index lesion can often be accurately localized and targeted.
\end{abstract}

METHODS: We performed a review of focal therapies for PCa, focusing on oncologic and functional outcomes.

RESULTS: Several modalities for focal therapy approaches are under investigation. Focal therapy of PCa is a rapidly developing area of research. In comparison with whole-gland treatment, it is clear that short- and intermediateterm functional and quality-of-life outcomes are excellent with focal therapies. However, only intermediate-term surrogate oncologic outcomes are available for specific focal treatments such as high-intensity focused ultrasound and cryoablation. For many other modalities such as irreversible electroporation, only follow-up biopsy information is available.

CONCLUSIONS: This review discusses the rationale for focal prostate treatment, describes the current state of the literature, and lists potential advantages and drawbacks to various therapeutic modalities.

\section{Introduction}

Prostate cancer (PCa) is the most common noncutaneous cancer in men in the United States. Approximately 191,930 men will be diagnosed and 33,330 men will die from PCa in 2020. ${ }^{1}$ With the introduction of widespread prostate-specific antigen (PSA) screening, most men are diagnosed at the localized PCa stag- es. Several well-established treatment options, such as radical prostatectomy, radiation therapy, and whole-gland cryotherapy, are available for localized PCa. Although these treatment modalities are highly effective in providing a cure at the localized stage, they are often associated with debilitating adverse effects (AEs) such as erectile dysfunction

\section{PERSPECTIVE}

Thomas J. Polascik, MD

considers the future of primary

focal therapy for localized

prostate cancer. page 269

and urinary incontinence. As a result, patients often delay or defer treatment of their PCa altogether.

In the past decade, active surveillance (AS) has increased for low- and very low-risk PCa, which has reduced overtreatment and sequelae of definitive treatment. However, as noted in the PROTECT trial, approximately $50 \%$ of AS patients will ultimately require treatment due to disease progression. ${ }^{2}$ Additionally, some patients will progress to the nonlocalized disease stages during the surveillance period and miss the opportunity for a cancer cure. Risk-stratification methods to better select and monitor patients for AS is an area of active investigation. Yet, for many patients, both the increased oncologic risks associated with AS and the quality-of-life concerns related to wholegland treatment are understandably unacceptable. As a result, the evolving literature around focal therapy has garnered much attention among patients and providers.

Focal treatment modalities treat the diseased area of the organ while 
preserving the normal surrounding tissue to preserve organ function and minimize AEs. Focal treatment of malignancies has been successfully implemented in breast, thyroid, lung, colon, and kidney cancers among others, with oncologic outcomes comparable with those of radical surgical resection. Recently, focal treatment modalities have been applied to PCa. Although PCa is largely a multifocal disease, a single focus of cancer, often called the index lesion, is thought to drive progression and to harbor the genetic precursor cells of advanced late-stage cancer. ${ }^{3}$ Pretreatment disease localization was previously a challenge in $\mathrm{PCa}$, but significant strides have been made in this area with the advances in multiparametric MRI (mpMRI) for diagnosis and staging. mpMRI is highly sensitive for high-grade $\mathrm{PCa}$ and for detecting the index lesion at rates of $72 \%$ and $80 \%$, respectively, on examining whole-mount pathology. With ultrasound fusion platforms, the disease area can be precisely localized and targeted with a transrectal or transperineal approach. Several devices that deliver ablative energies have become available to target the specific disease area for treatment. Standard-of-care options such as surgery and radiation are also being modified and subsequently investigated to evaluate the safety and efficacy of partial prostate treatment.

This article describes the available focal therapy options for primary treatment of localized PCa, along with their respective outcomes, drawbacks, and applicability.

\section{Methods}

We reviewed PubMed using multiple permutations of relevant search terms including but not limited to prostate cancer, focal therapy, focal ablation, focal high-intensity focused ultrasound (HIFU)" cryoablation, focal laser ablation, irreversible electroporation, photodynamic therapy, brachytherapy, radiofrequency ablation, partial prosta- tectomy, and focal transurethral ultrasound ablation (TULSA). We focused on the studies describing the primary treatment of PCa. Studies of salvage focal therapies were excluded. A fulltext review of all selected articles was performed.

\section{Results \\ Rationale for the Use of Focal Therapy}

In 2012, in response to increasing concerns over harms caused by $\mathrm{PCa}$ overtreatment, the US Preventative Services Task Force (USPSTF) discouraged the use of PSA screening to detect PCa. The USPSTF has since reversed its PSA screening position, due to longer follow-up of clinical trial patients that showed a decrease in number necessary to screen to prevent 1 death from $\mathrm{PCa}$, and due to more frequent adoption of AS to avoid overtreatment of indolent disease. Now the USPSTF makes a grade $\mathrm{C}$ recommendation for PSA screening based on shared decision-making between physicians and patients. Although increased adoption of AS for low-risk disease has decreased overtreatment and related AEs, the decision of any patient and their physician to cease AS and pursue definitive whole-gland treatment remains challenging. Discontinuation of AS occurs both because of progression to aggressive disease and because of patient and physician anxiety about untreated cancer. A clear unmet need exists for a more nuanced balance of treatment options, falling between AS and radical therapies, to optimally treat visible cancer with minimal AEs.

Between $13 \%$ and $38 \%$ of radical prostatectomy specimens contain a single focus of cancer. ${ }^{5}$ The histopathology of index lesions predicts PCa's natural history in the vast majority of patients. ${ }^{6}$ Advances in MRI technology, including improved protocols and the introduction of the 3 Tesla machine, allow better localization of PCa lesions. Whole-mount pathology of prostatectomy specimens has demonstrated concordance of the lesion with a visible lesion on MRI. ${ }^{4}$ Further, systematic biopsy combined with MRI-targeted biopsy allows optimal sampling of the prostate to determine the extent and multifocality of PCa. Image-guided localization enables investigators to target and treat these lesions with a multitude of ablative energies, radiation, and partial-gland surgery. These treatment modalities allow the preservation of anatomical structures sustaining continence and erectile function, thus minimizing AEs.

\section{Focal Therapy Treatment Modalities}

Several focal treatment modalities are available to selectively treat the disease area in the prostate. They differ in terms of energy source and their effects on the prostate tissue. Selection of the treatment modality for focal treatment of PCa depends on the availability of the focal treatment modality at the treatment center, training of the physician, size of the prostate, location of the index lesion, extent of disease outside the index lesion, calcifications in the prostate, and patient-specific factors. Widely used patient selection criteria, as well as common focal treatments, are summarized in the Figure.

Here, we summarize available treatment modalities, proposed mechanisms, and findings from the pivotal studies. Important oncologic outcomes from the studies are noted in the Table.

\section{Focal High-Intensity Focused Ultrasound}

HIFU uses high-energy ultrasound to target and ablate a parabolic-shaped area of prostate tissue. The prostate is imaged via a transrectal ultrasound probe, and the PCa lesion is mapped out. Ultrasound energy then induces cell necrosis by thermal energy, heating the tissue up to $100^{\circ} \mathrm{C} .^{7}$

Several devices are commercially available and have been studied, including 


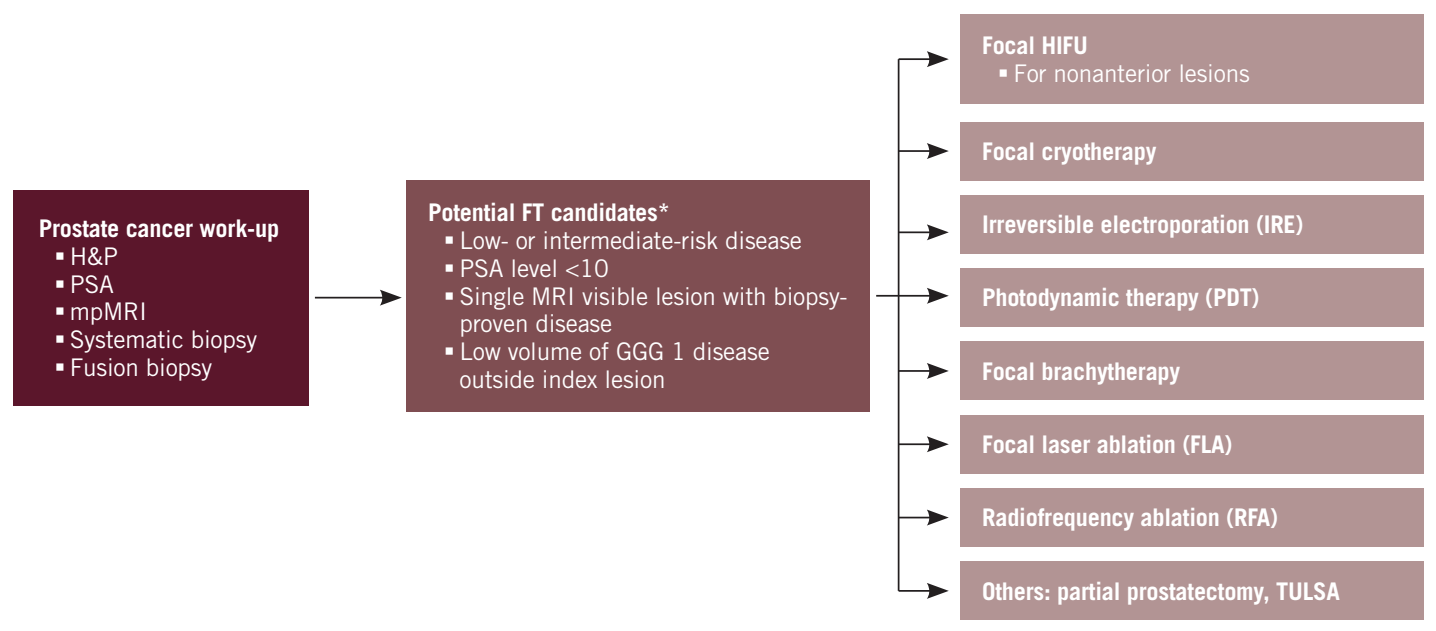

FT, focal therapy; H\&P, history and physical; HIFU, high-intensity focused ultrasound; GGG, Gleason grade group; mpMRI, multiparametric MRI; PSA, prostatespecific antigen; TULSA, transurethral ultrasound ablation

Ablatherm, Focal One, and Sonablate 500. HIFU has been studied in primary whole-gland treatment, salvage treatment, and primary focal therapy settings. National Comprehensive Cancer Network (NCCN) guidelines recommend salvage HIFU for radio-recurrent PCa. However, no guideline recommendations exist for the use of HIFU in the primary setting. The FDA approved HIFU for tissue ablation in 2015, but it was not approved for PCa treatment due to inadequate data for its efficacy in the primary setting.

A multicenter study reported 5-year outcomes for 625 men who received primary focal HIFU. Patients with localized Gleason 9 or less, cT3b or lower, and PSA $\leq 30 \mathrm{ng} / \mathrm{mL}$ were included in the study. The primary outcome was failure-free survival (FFS), a composite outcome of avoiding whole-gland therapy, systemic therapy, metastasis, and $\mathrm{PCa}-$ specific death. In the study, $83 \%$ of the cohort had Gleason 6 or 7 disease, and $75 \%$ had PSA $<10 \mathrm{ng} / \mathrm{mL}$. FFS was $88 \%$ at 5 years for all patients. In a prespecified subgroup analysis, FFS for low-, intermediate-, and high-risk patients was $96 \%, 88 \%$, and $84 \%$, respectively, and metastasis-free survival was $96 \%$, $99 \%$, and $97 \%$, respectively. The most common complication was urinary tract infection at a rate of $10.4 \%$. The most severe complication was rectourethral fistula formation in 2 patients $(0.3 \%)$, 1 of whom required reconstruction. At 2 to 3 years of follow-up, $80 \%$ of patients reported no urinary leakage at all, and $98 \%$ were pad-free. Erectile function and retreatment rates were not reported. ${ }^{8}$

Recently, Abreu and colleagues evaluated the use of hemigland HIFU in the primary setting in 100 men with localized PCa. By NCCN risk stratification, 20\% had low-risk, $50 \%$ had intermediate favorable, $17 \%$ had intermediate unfavorable, and $5 \%$ had high-risk disease. The 2-year survival rates specific for freedom from treatment failure, local recurrence of Gleason 7 or greater, repeat focal HIFU, and radical treatment were $73 \%, 76 \%$, $90 \%$, and $91 \%$, respectively. Of the men who underwent posttreatment biopsy, $17 \%$ had in-field recurrence and $14 \%$ had out-of-field recurrence with Gleason grade group 2 and higher. No declines in potency, measured by the International Index of Erectile Function (IIEF), were noted after treatment. Posttreatment continence was also excellent. ${ }^{9}$ In another study by Nahar and colleagues, 52 patients were treated with primary focal HIFU, of whom $67 \%$ harbored Gleason score 7 or higher. In-field (17\%) and out-of-field recurrences $(13 \%)$ were noted. Approximately $10 \%$ of patients had grade 3 or higher complications. In all patients with available data, urinary and sexual function returned to baseline at 3 to 6 months and at 12 months, respectively. ${ }^{10}$

Based on the available short- to intermediate-term studies, HIFU is marked by good efficacy, low rates of in-field and out-of-field recurrences, and preservation of urinary and sexual function. Several limitations are noted for focal HIFU, however. The patient must be free of limiting rectal pathology that would preclude use of a transrectal probe for delivery. Large glands $>40$ g may be a contraindication for HIFU using some older devices, as the focal length may not 
be adequate. ${ }^{11}$ Anterior lesions treated with primary HIFU have been shown to require additional treatment at a higher rate than posterior lesions, as the penetration depth of the ultrasound energy is limited. ${ }^{12}$ Widespread calcifications may also be a limitation; they can cause acoustic shadowing, which does not allow passage of the therapeutic sound waves. Further efforts are under way to identify patients who will fail the focal HIFU treatment, so they may be offered a different focal modality or whole-gland treatment.

\section{Focal Cryoablation}

Cryoablation is rapid cooling performed by transperineal needle placement down to temperatures of $-40^{\circ} \mathrm{C}$. It results in the formation of an ice ball and subsequent tumor lysis. Preclinical trials suggested that margins of at least $1 \mathrm{~cm}$ around the target tissue are needed to ensure cancer ablation. ${ }^{13}$

Most of the data for the focal prostate cryoablation studies come from the COLD (Cryo On-Line Data) registry. The largest prospective registry of focal cryoablation reported early outcomes of 122 men; largely, they had intermediate-risk disease, confirmed with mpMRI targeted biopsy or transperineal mapping, with a maximum cancer core length of $6 \mathrm{~mm}$. These patients were explicitly selected due to unsuitability for HIFU (because of anterior disease, anterior-posterior height of greater than $3 \mathrm{~cm}$, or widespread calcifications). Standard FFS was the primary outcome, at a rate of $90.5 \%$ at 3 years. Stratified by risk, FFS was better in intermediate-risk disease $(93 \%)$ compared with high-risk disease $(84 \%)$. A total of $6.5 \%$ required additional focal cryotherapy at last follow-up. In a subgroup analysis of 2 groups stratified by PSA, those with PSA $<10 \mathrm{ng} / \mathrm{mL}$ had higher FFS $(97 \%)$ compared with patients with PSA > $10 \mathrm{ng} / \mathrm{mL}(84 \%) .{ }^{14}$

Complications were reported in $27.8 \%$ of men; urinary tract infection and temporary urinary retention were the most common. Serious complications included osteomyelitis in 1 patient who was managed conservatively, and no rectourethral fistulas were reported. At 3 years, 87\% and $89 \%$ had returned to baseline International Prostate Symptom Scores (IPSS) and IIEF scores, respectively. ${ }^{15}$

Another prospective single-institution registry of 119 men who received primary focal cryotherapy reported slightly lower FFS, $73 \%$ and $56 \%$ at 3 and 5 years, respectively. Radical treatment-free survival was also reported, at rates of $83 \%$ and $70 \%$ at 3 and 5 years, respectively. ${ }^{16}$ Similar outcomes have been noted with hemigland ablation. In a retrospective study of 160 patients with largely intermediate-risk cancer, FFS without retreatment was $85 \%$ at 5 years. High baseline PSA was predictive of failure. ${ }^{17}$

Cryotherapy is not the first energy modality of choice for posterior or apical tumors, as surrounding tissue damage can occur, affecting the neurovascular bundles and subsequently erectile function. ${ }^{18}$ As such, patient selection is critical in the success of focal cryotherapy. Similarly, cryotherapy may also not be well suited for small glands, as the expanding ice ball may extend beyond the prostate. ${ }^{18}$ However, although these preferences for tumor location and prostate size are repeatedly seen in the literature, trials with well-defined selection criteria do not support or refute those preferences.

\section{Photodynamic Therapy}

Photodynamic therapy (PDT) uses oral or intravenous drugs that are inactive until they are photosensitized, at which time they form reactive oxygen species leading to tissue destruction. A laser fiber is targeted at the prostate, which is typically placed using transrectal ultrasound guidance. Several types of photosensitizing drugs are used and are usually categorized as either tissue-based or vascular-based, depending on where the drugs are activated. The drugs can take up to several days to reach maximum dose in the tissue, while vascu- lar-based drugs activate within minutes after photosensitization. ${ }^{19}$

In a large phase 3 randomized controlled trial, patients with low-risk PCa were randomized to either AS or padeliporfin vascular-targeted PDT. The median follow-up was 24 months and 206 men were randomized to PDT. The primary outcome was time to progression, a composite outcome of having 4 or more positive cores, any Gleason pattern 4 or greater, at least 1 cancer core length greater than $5 \mathrm{~mm}, \mathrm{PSA}>10 \mathrm{ng} / \mathrm{mL}$ on 3 consecutive measures, any T3 PCa, or metastasis. The study measured the median time for the PCa to progress from low-risk to moderate-risk or high-risk; this time was longer in the PDT group than the AS group, at 28.3 months vs 14.1 months $(P<.0001)$. When compared with the AS group, more men in the PDT group had negative biopsies at 24 months (49\% vs $14 \% ; P<.0001)$ and fewer men in the PDT group underwent radical therapy of either radiation or surgery $(6 \%$ vs $29 \%$; $P<.0001)$. Erectile and urinary function were similar between the 2 groups at 24 months. AEs in the PDT group were pain, urethral stricture, urge incontinence, and urinary retention. ${ }^{20}$

Other studies of relatively small cohorts have shown similar short-term outcomes. In one prospective trial, 82 men with low-risk PCa were treated with PDT. The primary end point was progression-free survival (PFS), defined as an intermediate-risk or high-risk group by D'Amico classification or radical therapy. Median PFS was 86 months, and only $24 \%$ of patients underwent radical treatment. ${ }^{21}$

Overall, PDT is effective in delaying the progression of low-risk cancer compared with AS. Potential drawbacks to PDT include the need to avoid direct sunlight following the procedure for 48 hours to reduce AEs and the potential of an allergic reaction to the photosensitized agent.

\section{Irreversible Electroporation}

Irreversible electroporation (IRE) uses 
TABLE. Oncologic Outcomes of Focal Therapies by Modality

\begin{tabular}{|c|c|c|c|c|c|c|c|}
\hline Mode & Authors and year & $\begin{array}{l}\text { Prospective }(p) \text { or } \\
\text { retrospective }(r)\end{array}$ & Patients & $\begin{array}{l}\text { Highest } \\
\text { GGG }\end{array}$ & Follow-up & Primary outcome & Rate of outcome \\
\hline \multirow{3}{*}{ Focal HIFU } & Guillamier et al (2018) & $\mathrm{p}$ & 625 & 2 & $56 \mathrm{mo}$ & FFS & $88 \%$ at $5 y$ \\
\hline & $\begin{array}{l}\text { Tourinho-Barbosa et al } \\
(2020)\end{array}$ & $\mathrm{p}$ & 190 & 3 & $45 \mathrm{mo}$ & $\mathrm{FFS}^{\mathrm{a}}$ & $54 \%$ at $5 y$ \\
\hline & Abreu et al (2020) & r & 100 & 4 & $18 \mathrm{mo}$ & FFS & $73 \%$ at $2 \mathrm{y}$ \\
\hline \multirow{3}{*}{ Focal cryotherapy } & Shah et al (2019) & $\mathrm{p}$ & 122 & 3 & $28 \mathrm{mo}$ & FFS & $\begin{array}{l}93 \% \text { for intermediate } \\
\text { risk and } 85 \% \text { for high } \\
\text { risk at } 3 y\end{array}$ \\
\hline & $\begin{array}{l}\text { Tourinho-Barbosa et al } \\
\text { (2020) }\end{array}$ & $\mathrm{p}$ & 119 & 3 & $45 \mathrm{mo}$ & $\mathrm{FFS}^{\mathrm{a}}$ & $56 \%$ at $5 y$ \\
\hline & Oishi et al (2019) & r & 160 & 5 & $40 \mathrm{mo}$ & TFS & $85 \%$ at $5 y$ \\
\hline \multirow[t]{2}{*}{ Focal PDT } & Azzouzi et al (2017) & $\mathrm{p}$ & 206 & 1 & $24 \mathrm{mo}$ & Treatment failure & $\begin{array}{l}28 \% \text { at } 24 \text { mo } \\
\text { (compared with } 58 \% \\
\text { in active surveillance) }\end{array}$ \\
\hline & Lebdai et al (2017) & $\mathrm{p}$ & 82 & 1 & $68 \mathrm{mo}$ & PFS & $76 \%$ at $80 \mathrm{mo}$ \\
\hline \multirow[b]{2}{*}{ Focal IRE } & Blazevski et al (2020) & $\mathrm{p}$ & 123 & 3 & $36 \mathrm{mo}$ & FFS & $97 \%$ at $3 \mathrm{yr}$ \\
\hline & Scheltema et al. (2018) & $r$ & 50 & 3 & $12 \mathrm{mo}$ & $\begin{array}{l}\text { Residual csPCa at } \\
\text { follow-up biopsy }\end{array}$ & $29.5 \%$ at $12 \mathrm{mo}$ \\
\hline \multirow[b]{2}{*}{ HDR brachytherapy } & Peters et al (2019) & $\mathrm{p}$ & 30 & 3 & $48 \mathrm{mo}$ & BCDFS & $70 \%$ at $4 \mathrm{y}$ \\
\hline & King et al (2018) & $r$ & 354 & 2 & $8.6 \mathrm{y}$ & Metastasis, PCSM & $\begin{array}{l}15.7 \% \text { and } 8.2 \% \text { at } \\
12 \mathrm{y} \text { (intermediate } \\
\text { risk only) }\end{array}$ \\
\hline \multirow{2}{*}{ FLA } & Eggener et al (2016) & $\mathrm{p}$ & 27 & 2 & $12 \mathrm{mo}$ & $\begin{array}{l}\text { Residual cancer at } \\
\text { follow-up biopsy }\end{array}$ & $\begin{array}{l}37 \% \text { at } 12 \mathrm{mo}(11 \% \\
\text { in field) }\end{array}$ \\
\hline & Chao et al (2018) & $\mathrm{p}$ & 34 & 3 & $24 \mathrm{mo}$ & $\begin{array}{l}\text { Residual cancer at } \\
\text { follow-up biopsy }\end{array}$ & $\begin{array}{l}40 \% \text { at } 24 \mathrm{mo} \text { (all in } \\
\text { field) }\end{array}$ \\
\hline Focal RFA & Orczyk et al (2020) & $\mathrm{p}$ & 20 & 3 & $12 \mathrm{mo}$ & $\begin{array}{l}\text { Residual csPCa at } \\
\text { follow-up biopsy }\end{array}$ & $\begin{array}{l}20 \% \text { at } 6 \mathrm{mo} \text { (all in } \\
\text { field) }\end{array}$ \\
\hline $\begin{array}{l}\text { Partial } \\
\text { prostatectomy }\end{array}$ & Villers et al (2017) & $\mathrm{p}$ & 17 & 3 & $24 \mathrm{mo}$ & $\begin{array}{l}\text { Local recurrence-free } \\
\text { survival }\end{array}$ & $67 \%$ at $3 y$ \\
\hline Focal TULSA & Antinnen et al (2019) & $\mathrm{p}$ & 6 & 4 & $3 w k$ & $\begin{array}{l}\text { Histological } \\
\text { destruction of tissue }\end{array}$ & $100 \%$ in field \\
\hline
\end{tabular}

BCDFS, biochemical disease-free survival; csPCa, clinically significant prostate cancer; FFS, failure-free survival; FLA, focal laser ablation; GGG, Gleason grade group; HDR, high dose rate; HIFU, high-intensity focused ultrasound; IRE, irreversible electroporation; PCSM, prostate cancer-specific mortality; PDT, photodynamic therapy; PFS, progression-free survival; RFA, radiofrequency ablation; TFS, treatment-free survival; TULSA, transurethral ultrasound ablation.

alncludes retreatment.

microsecond electrical pulses to generate pores in the cell membrane. While reversible electroporation allows for temporary changes to the cell, IRE leads to cell destruction as the cells cannot maintain homeostasis. Compared with other ablative modalities, IRE appears to spare surrounding nerves and vessels from collateral damage. ${ }^{22}$ In theory, this property suggests that IRE is better suited for focal treatment of PCa compared with other ablative modalities. During IRE of the prostate tumor, patients are placed in the lithotomy position under general anesthesia with full muscle paralysis to reduce muscle spasms. Needle probes are placed transperineally, typically using a brachytherapy template. ${ }^{23}$

Valerio and colleagues studied the safety and feasibility of focal IRE. A total of 34 patients, primarily with lowand intermediate-risk $\mathrm{PCa}$, underwent focal IRE. No grade 3 complications were noted. At 6 months follow-up, from a functional perspective, $100 \%$ of men were continent and $95 \%$ were potent after focal IRE. Approximately $20 \%$ had residual cancer, as evaluated by mpMRI. ${ }^{24}$ In a phase $1 / 2$ study, van den Bos and colleagues evaluated the histopathology of the prostate tissues of men who underwent IRE, and all cells within the electrode zone were completely 
ablated. No skip lesions were noted, demonstrating the effectiveness of the IRE as an ablation modality. ${ }^{25}$

Blazevski and colleagues evaluated their experience with IRE in 123 men with $\mathrm{PCa}$, a majority $(91 \%)$ with intermediate-risk disease, at a median follow-up of 3 years. FFS, defined by whole-gland treatment, systemic disease, or death, was $96 \%$ at 3 years. In up to $9 \%$ of the patients, in-field recurrences were noted. Eighteen men required retreatment: 12 underwent repeat IRE and 6 had whole-gland treatment. Functional outcomes were excellent, with $98 \%$ of men remaining pad free and $76 \%$ having no change in erectile function. ${ }^{26}$

There are no randomized trial comparisons of IRE with radical prostatectomy in the literature. However, Scheltema and colleagues made a pair match comparing 50 men with cT1c-T2b, unifocal disease who underwent IRE with 50 men who underwent robot-assisted nerve-sparing radical prostatectomy. No significant differences emerged in the baseline oncologic and functional characteristics. At 12 months, urinary continence and sexual function were superior in the men who underwent IRE treatment compared with those who had robot-assisted laparoscopic radical prostatectomy (RARP), but higher local failure $(29.5 \%)$ was noted in the men treated with IRE. ${ }^{27}$

IRE is a promising focal therapy treatment. However, further larger studies with appropriate control arms are needed to establish its role in PCa treatment, as well as to define potential selection criteria and drawbacks.

\section{Focal Brachytherapy}

Whole-gland brachytherapy and salvage brachytherapy have been used to treat localized PCa and have been recommended in the NCCN guidelines to treat low-risk disease. However, little evidence exists for primary MRI-guided brachytherapy for focal therapy of PCa. Although the field has anticipated the promise of focal high dose rate (HDR) brachytherapy for PCa, clinical trials have not, in many cases, delivered promising outcomes..$^{28}$

King and colleagues reviewed their experience with focal MRI-guided brachytherapy in a large cohort of 354 men: $40 \%, 39 \%$, and $21 \%$ of patients had very low-risk, low-risk, and intermediate-risk disease, respectively. At a median of 11 years follow-up, 22 men $(6.2 \%)$ had developed metastatic disease. The 12-year metastasis rates for patients with very low-risk, low-risk, and intermediate-risk disease were $0.8 \%$, $8.7 \%$, and $15.7 \%$, respectively.

More importantly, the 12 -year PCa-specific mortality estimates were $1.6 \%$ in very low-risk, $1.4 \%$ in low-risk, and $8.2 \%$ in intermediate-risk disease, respectively. Due to the high rate of metastasis and $\mathrm{PCa}$-specific mortality rates, the authors concluded that partial gland ablation with brachytherapy might not be appropriate for patients with intermediate-risk disease. ${ }^{29}$

Other prospective studies with shorter-term follow-up have shown concordant results. Biochemical recurrence-free survival after focal HDR brachytherapy was poor in one study with 4 years of follow-up, at a rate of $70 \%$ in 30 patients. ${ }^{30}$ No other relevant oncologic outcomes, such as FFS or salvage whole-gland treatment, were reported in this study.

\section{Focal Laser Ablation}

Focal laser ablation (FLA) is an MRI-guided technique that uses a low-powered laser fiber to deliver thermal energy transrectally to the soft-tissue target. The tissue ablation temperature exceeds $60^{\circ} \mathrm{C}$. This technology was adapted from use in intracranial neoplasms. ${ }^{31}$

Only a few small studies have reported outcomes from use in the primary treatment of PCa. One phase 2 trial of 27 men, primarily with low-risk disease, received FLA for up to 2 MRI-visible lesions. No residual cancer was noted in $96 \%$ of men, utilizing MRI-targeted biopsy 3 months after treatment, but residual cancer was detected in $37 \%$ at a fol- low-up systematic biopsy at 12 months. The complication profile was favorable, with hematuria, perineal ecchymosis, and urinary retention as the most common AEs. ${ }^{32}$ No significant difference was seen in IPSS or Sexual Health Inventory for Men (SHIM) scores between baseline and at 12 months. In another study by Lepor and colleagues, 25 men with Gleason score $<8$, cT1c-T2a, and PSA $<10 \mathrm{ng} / \mathrm{mL}$ were treated with FLA and monitored with MR thermometry. No decline in IPSS or SHIM score was noted at 3 months, with $96 \%$ of men noted to have a negative ablation-zone biopsy. ${ }^{33}$

In a larger prospective trial of $120 \mathrm{pa}-$ tients who underwent FLA for primarily low- or intermediate-risk disease, $15 \%$ harbored residual clinically significant disease upon follow-up MRI-targeted biopsy; $17 \%$ required retreatment of some sort. A change in technique significantly limited this study during the study period. ${ }^{34}$ Two-year oncologic outcomes were reported in another prospective study of primary FLA, and $18.8 \%$ harbored clinically significant $\mathrm{PCa}$ at follow-up biopsy. ${ }^{35}$

Prostate volume limitations and contraindications to FLA are not well defined in the literature. ${ }^{36}$

\section{Focal Radiofrequency Ablation}

Focal radiofrequency ablation (RFA) has been studied as a treatment for cancer in various organs, including the liver and kidney. It delivers thermal energy using medium-frequency alternating current by way of a transperineal probe. ${ }^{37}$

Only 1 prospective phase 2 study exists in the literature. It recently reported the short-term outcomes of 20 men who underwent bipolar RFA, $90 \%$ of whom had Gleason 7 disease. At a 6-month follow-up targeted biopsy, $80 \%$ of men were free of clinically significant PCa within the treated lesion. Utilizing the Expanded Prostate Cancer Index Composite questionnaire, $89 \%$ of patients who had no baseline urinary dysfunction were found to be free of incontinence at 
12 months. Using responses to the IIEF, the investigators found that $91.7 \%$ of patients with no baseline erectile dysfunction reported the ability to achieve adequate erections with or without phosphodiesterase-5 inhibitors. ${ }^{38}$ No serious AEs were reported.

Due to the paucity of literature for focal RFA in this setting, additional research is needed to establish selection criteria and drawbacks.

\section{Partial Prostatectomy}

Partial prostatectomy is a subtotal surgical resection of a prostate tumor, and cancer control outcomes of this approach have been sparsely recorded in the literature. However, recently, the short-term results of a single-arm multicenter prospective trial of robotic partial prostatectomy for anterior tumors were reported. ${ }^{39}$ Seventeen patients, primarily with anterior preurethral tumors visible on MRI, were enrolled. All patients underwent robotic partial prostatectomy via a transperitoneal approach. Perioperative outcomes were excellent, with no grade 3 complications reported. Three-month continence and erectile function rates were $100 \%$ and $83 \%$, respectively. Long-term oncologic outcomes were not reported. However, the local recurrence-free survival rate was $67 \%$ at 3 years, and $24 \%$ of men underwent a completion radical prostatectomy.

Although this is a novel approach for the treatment of anterior zone PCa, only a few men would be eligible to meet the inclusion criteria of preurethral tumors, and with a high local failure rate, some would require whole-gland therapy soon after.

\section{Focal Transurethral Ultrasound Ablation}

MRI-guided TULSA is a novel procedure in which prostate tissue is ablated in a continuous sweeping fashion by the directed ultrasound. The ablation zone is monitored with MR thermometry. It has been studied mainly as a whole-gland therapy for PCa.
Recently, the TULSA-PRO (designated for the technology's manufacturer, Profound Medical) Ablation Clinical Trial protocol was developed, and the single-arm multicenter trial was completed for the whole-gland treatment of PCa. Patients with Gleason 6 and 7, cT1 to cT2b, and MRI visible tumors, with PSA $<15 \mathrm{ng} / \mathrm{mL}$, were enrolled in the study. Patients with prostate volume $>90 \mathrm{cc}$, intraprostatic calcifications $>1 \mathrm{~mm}$, and prostate tumors within 3 $\mathrm{mm}$ of the prostate apex on MRI were excluded. The trial's primary objectives were safety and efficacy, defined as PSA decline equal to or greater than $75 \%$. The secondary oncologic objective was disease improvement at repeat biopsy, defined as no cancer or reduced cancer grade on 10-core biopsy at 12 months. Urinary function was evaluated with IPSS, and erectile dysfunction was evaluated with the IIEF score. A total of 115 men, 33\% with low-risk disease and 67\% with intermediate-risk disease, underwent whole-gland therapy. At 12 months, 8\% of patients had grade 3 complications, with urinary tract infection and urethral stricture being the most common. A total of $96 \%$ of patients met the primary objective of PSA decline $>75 \%$. A total of $23 \%$ of patients developed moderate erectile dysfunction requiring medical management. No cancer was noted at 12-month biopsy in $75 \%$ to $80 \%$ of men. The risk factors for treatment failure were noted to be intraprostatic calcification, failure to achieve thermal dose coverage $>96 \%$, and Prostate Imaging-Reporting and Data System >3 lesion at 12 months. ${ }^{40}$

TULSA-PRO is awaiting FDA approval, and further studies are under way to study its role in the focal therapy of PCa. The results of multiple treat-then-resect studies have been reported, indicating the safety and potential efficacy of focal TULSA. A phase 1 study investigated the feasibility of MRI-guided TULSA. Six men with 1 to 2 MRI-visible lesions far from the neurovascular bundles were enrolled. No treatment-related complica- tions were reported in the 3 weeks leading up to RARP. At surgical pathology, no residual in-field cancer was seen. ${ }^{41}$

\section{Discussion}

Treatment of localized $\mathrm{PCa}$ reduces the risks of progression and $\mathrm{PCa}$-specific death, but significant adverse long-term functional outcomes of prostatectomy and radiation therapy for localized $\mathrm{PCa}$ treatment have been well characterized. Up to $18 \%$ of patients report significant bother from urinary leakage, and 43\% are bothered by sexual dysfunction at 15 years. ${ }^{42}$ AS may preserve functionality for some well-selected patients at the cost of a higher risk of progression before definitive treatment can be performed. For many intermediate-risk patients for whom the risk of progression with AS is unacceptably high, but for whom quality of life with whole-gland treatment would be too low, focal therapy offers a possible solution. This potential benefit with regard to functional preservation is well supported by the data we have discussed. Incontinence and erectile dysfunction rates for focal therapy are milder in both the maximum degree and duration of recovery when compared with rates for whole-gland therapy. Even in the most contemporary nerve-sparing robotic prostatectomy series, severe incontinence and erectile dysfunction are reported at detectable rates that are not seen in any of the focal therapy cohorts. ${ }^{43}$

However, many questions remain unanswered for every mode of focal therapy for PCa. The longest-term oncologic outcomes are 5 years for HIFU and cryoablation. Metastasis-free and cancer-specific survival are very high, as would be expected with intermediate-term outcomes. Radical whole-gland treatment is avoided in most patients, and FFS is as high as $88 \%$ in men with intermediate-risk disease 5 years following focal HIFU. ${ }^{8}$ Similarly, high radical treatment-free survival is seen with focal cryotherapy over a medium-term period, but it has been reported as low as $70 \%$ 
at 5 years. Many patients receiving both cryotherapy and HIFU will need repeat focal treatment for definitive cancer control. ${ }^{44}$ The majority of patients who undergo focal treatment avoid radical therapy, likely at a rate lower than those with intermediate-risk disease who initially pursue AS in contemporary protocols. ${ }^{2,45}$ However, to our knowledge, only 1 randomized trial comparing any focal therapy to AS has reported this outcome, and this was for a low-risk cohort. ${ }^{28}$ As for energy modalities beyond HIFU and cryoablation, it is unclear at this point whether similar intermediate-term outcomes will be seen.

Other considerations are the outcomes of patients who require salvage treatment after focal therapy, our understanding of which is limited to small retrospective series. Marra and colleagues conducted a systematic review of short-term oncologic and functional outcomes following radical salvage treatment after focal therapy; they found little significant difference between outcomes following primary radical treatment, but the overall quality of evidence was low. ${ }^{46}$ Another more recent series of 45 men who underwent post-HIFU prostatectomy found that one-third of men required either additional radiation or androgen deprivation therapy at 12 months postoperatively, and only $65 \%$ were pad free, suggesting worse outcomes compared with primary prostatectomy. ${ }^{47}$

Patient selection criteria for focal therapy continue to evolve as the literature matures. Various cancer risk groups have been evaluated in many of the above studies, and as would be expected, highrisk patients do not fare as well as the patients with intermediate- or low-risk disease. ${ }^{48}$ Ideal tumor location has been widely described but poorly studied. A recent retrospective article comparing focal HIFU outcomes for anterior tumors and posterior tumors found that anterior location was predictive of treatment failure on multivariate analysis. ${ }^{12}$ This has been postulated in the literature previously. Prostate swelling during treatment can shift target lesions, and this effect has been described as having an exaggerated effect on anterior lesions. ${ }^{13}$ Comparable tumor location studies for cryotherapy have not been published. However, posterior tumors are thought to be unfavorable for this modality of treatment, due to collateral tissue destruction of the neurovascular bundle that is not seen with focal HIFU. ${ }^{13}$ The effects of other clinical factors have been described as well, such as PSA $>10 \mathrm{ng} /$ $\mathrm{mL}$; this has been demonstrated as a risk factor for treatment failure in multiple studies. ${ }^{48}$

In the AS era, a Delphi consensus statement has claimed that the ideal focal therapy candidate is a patient with a small unifocal mpMRI-visible lesion with biopsy-proven intermediate-risk disease and a maximum volume of $3 \mathrm{~mL}$ if confined to a single hemigland. ${ }^{48}$ What is known is that due to the intensive fol- low-up imaging and biopsies necessary, and the possible need for retreatment in most protocols, a high level of long-term patient adherence is required. Current guidelines do not recommend primary focal therapy outside the context of a clinical trial.

\section{Conclusions}

Focal therapy for PCa could balance undertreatment and overtreatment of localized PCa for highly selected patients. Long-term oncologic outcomes are not yet available for any modality. Intermediate-term oncologic outcomes appear promising for HIFU and cryoablation, and functional outcomes are superior to those of any form of whole-gland therapy. Patient selection remains key in the success of focal treatment modalities. Patients should be informed regarding currently available outcomes, the necessity of adherence to a stringent follow-up protocol, and the possible need for additional targeted therapy or future radical treatment in case of recurrence.

FINANCIAL DISCLOSURE: The authors have no significant financial interest in or other relationship with the manufacturer of any product or provider of any service mentioned in this article.

For full reference list, visit cancernetwork.com/Hayes_5.21

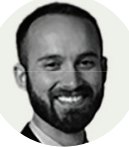

Portland, OR.

\section{Hayes}

is a resident physician in the Department of Urology at Oregon Health \& Science University in

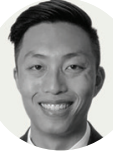

Portland, OR
Lin-Brande

is a resident physician in the Department of Urology at Oregon Health \& Science University in

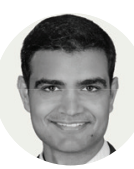

Portland, OR.

\section{Isharwal}

is an assistant professor in the Department of Urology at Oregon Health \& Science University in

Portland, OR. 


\section{Prostate Cancer Focal Therapy Has Made Great Strides and the Future Remains Bright}

A Ithough partial-gland therapies, also known as focal therapies, have been incorporated into the surgical treatment pathway of nearly every solid organ system, the prostate has been particularly challenging due mainly to 2 concerns: cancer multifocality and the difficulties of accurately imaging the boundaries of a distinct tumor(s) as typically occurs in other organs, such as the kidney. Despite these challenges, however, the slow but steady acceptance of the concept of focal therapy for prostate cancer has been growing over the past 2 decades, with gratitude to the dedicated individuals who have had the foresight to propel focal therapy to eventually become a standard treatment for select men. Support for the development of image-targeted interventions is also extended to our industry partners and collaborative scientists who continue to push the envelope on technical enhancements and new discoveries that aid in the detection, characterization, and image-guided treatment of such neoplasms.

With the widespread adoption of multiparametric $\mathrm{MRI}$, we now have a reliable imaging platform to direct patient selection and provide intraprocedural guidance and posttherapy surveillance. ${ }^{1} \mathrm{MRI}$ still leaves room for improvement, though, as up to $15 \%$ of tumors are MRI-invisible and the 3dimensional shape of each cancer with precise determination of the boundaries leaves room for discovery., Also, over the past 2 decades, physicians and patients have become more comfortable with the management of multifocality, provided that untreated, small, and indolent satellite tumors can be confidently monitored and do not pose a threat to a patient's life expectancy.
As the authors discuss, a number of short- to medium-term outcomes have been reported on primary focal therapy, ranging from single-institution and multicenter series to small clinical trials. ${ }^{4}$ From a functional point of view, most would agree that the results of focal therapy overall have been exceptional at preserving urinary function and continence. Potency outcomes have also been very competitive with, if not better than, traditional whole-gland therapies. However, cancer control has yet to demonstrate mature long-term data. Regarding oncologic outcomes, the reality is that even traditional therapies such as radiation and surgery have failure rates and certainly have adverse effects on the quality of life of many men. We should not expect focal therapy to improve the failure rate when compared with whole-gland therapy. Since it is a partial-gland therapy, a consensus panel believed that retreatment rates up to $20 \%$ with focal therapy were clinically acceptable. ${ }^{5}$ Further therapy may be required if a targeted ablation does not control a clinically significant tumor, and other men may require additional treatment if a de novo, or out-of-field, tumor is subsequently detected and deemed in need of therapy during follow-up.

Several ablative devices are on the market, some of which combine physical ablation with targeted drug/device therapy. This takes on yet another challenge when determining efficacy of focal therapy, as new devices and treatments will similarly be introduced over time. We need a means to address the effectiveness of these treatments, particularly in situations where the natural history of prostate cancer is often prolonged, requiring in excess of 10 years to determine oncologic outcomes. For the most part, clearly functional outcomes can be assessed in the short term, and the data to date have been very supportive of focal ablation. $^{4,5}$

Patient selection remains the cornerstone of properly applied focal therapy. With the well-chosen tumor/patient, many will be able to avoid radical treatment in the intermediate term while preserving genitourinary function. If this goal can be realized, it should be understood that a limited percentage of men may require an additional ablation at some point that may be reasonable as long as the cancer can continue to be controlled and quality of life maintained. We have already seen this in the renal literature, where a small percentage of patients will require a second ablation for treatment of a small T1a renal cell carcinoma, and this has been accepted by most practitioners and patients.

As we move forward with collecting long-term cancer outcomes, it is important to be mindful that patient and tumor selection remain the cornerstone of successful partial-gland ablation. Finally, it is important to be forthcoming with patients regarding the intended goals, benefits, and limitations of focal therapy, and to have them understand that retreatment, identification of de novo tumors, or progression is always possible.

FINANCIAL DISCLOSURE: The authors have no significant financial interest in or other relationship with the manufacturer of any product or provider of any service mentioned in this article.

For full reference list, visit cancernetwork.com/Polascik_5.21 


\section{IN A WORLD FILLED WITH COVID- 19 ...}

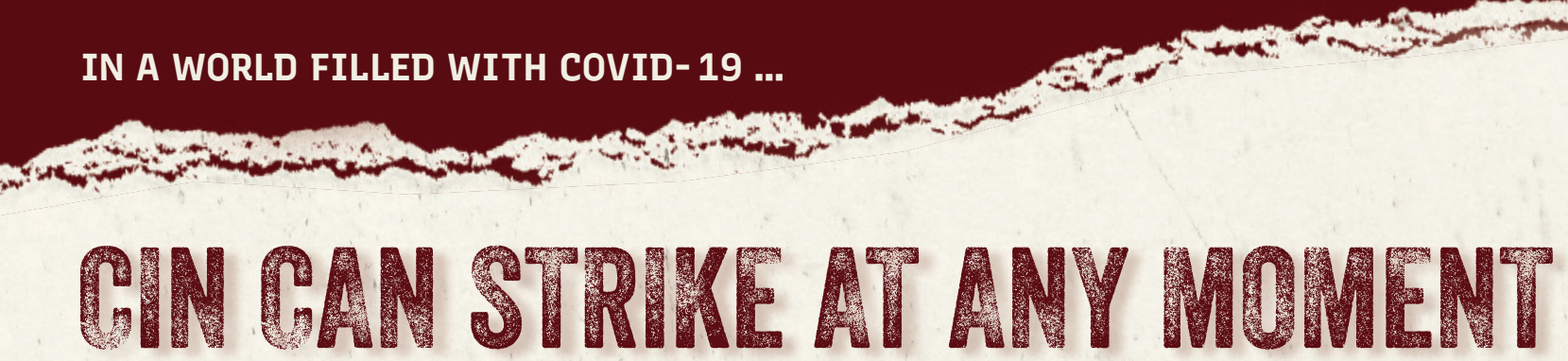

It's time to take a critical fresh look at chemotherapy-induced neutropenia (CIN), and how the COVID-19 pandemic is changing the standard-of-care guidelines.

Cancer plus COVID-19-and the threat patients everywhere are now facing

Infection has always been a concern in patients who may be at risk of neutropenia associated with chemotherapy. And now, with the onset of the global COVID-19 pandemic, they are facing an even higher mortality rate. While cancerassociated risk factors with COVID-19 are not yet fully understood, individuals with active hematologic or lung malignancies, lymphopenia, or baseline neutropenia show worse outcomes when also diagnosed with COVID-19. ${ }^{1}$ The result? A revisiting of practice guidelines, the expansion of prophylactic granulocytecolony stimulating factor (G-CSF) for patients who are at intermediate risk for neutropenia, and a renewed urgency to address the high unmet need of preventing CIN. ${ }^{2}$

When ANC drops, so do your patient's defenses

During chemotherapy, the absolute neutrophil count (ANC) drops until it reaches its lowest point (nadir). The timely detection and diagnosis of CIN make it possible to administer the appropriate G-CSF. ${ }^{3}$ Otherwise, severe (Grade 4) neutropenia may cause dose reductions in therapy regimens and compromise the chemotherapy's efficacy, potentially reducing a patient's survival. ${ }^{4}$ Fever and systemic infections from neutropenia are also serious complications (Figure 1), as they may lead to hospitalizations, which place substantial economic costs and strains on the healthcare system. ${ }^{5-8}$

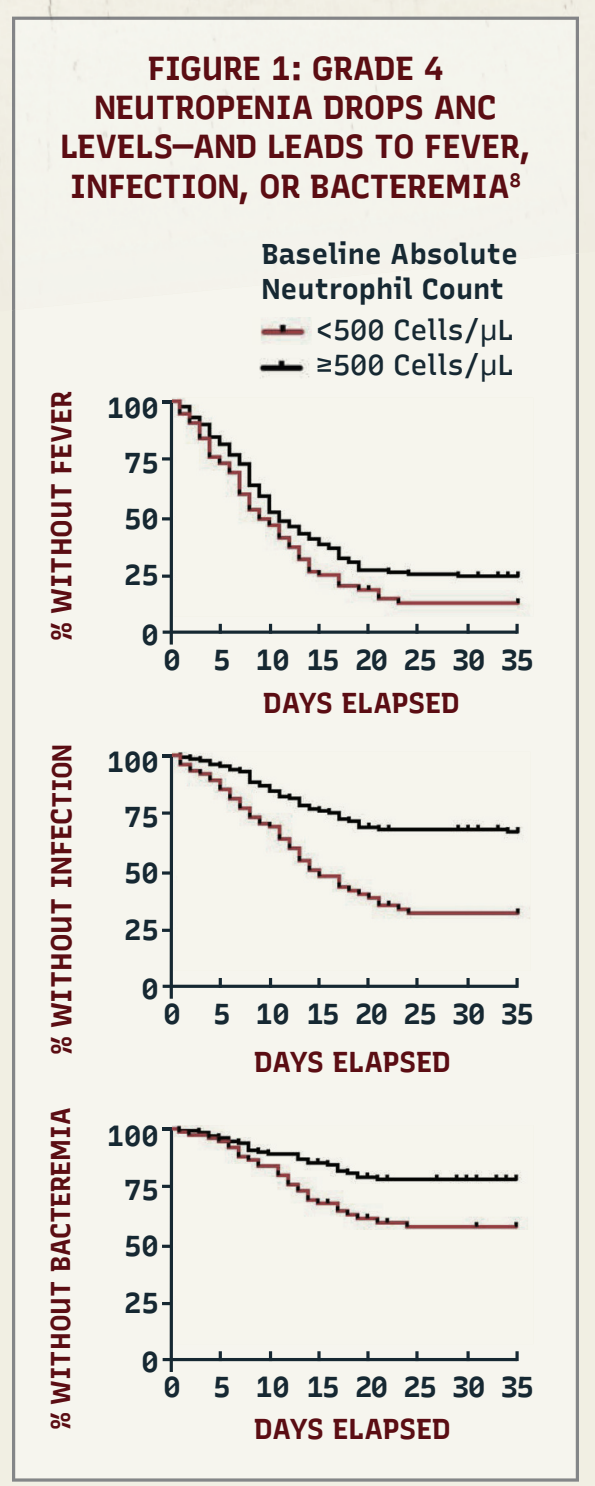

Republished with permission of [WILEY], from Buckley, S.A., Othus, M., Vainstein, V., Abkowitz, J.L., Estey, E.H. and Walter, R.B. (2014), Prediction of adverse events during intensive induction chemotherapy for acute myeloid leukemia or high-grade myelodysplastic syndromes. Am. J. Hematol., 89: 423-428. https://doi.org/10.1002/ ajh.23661; permission conveyed through Copyright Clearance Center, Inc.

There is a direct association between baseline blood counts and the likelihood of developing a fever or being diagnosed with a documented infection or bacteremia. ${ }^{8}$
Once regimens are disrupted due to low ANC levels, the eruption of complications begin

CIN remains the most common serious toxicity of current chemotherapeutic regimens. Despite advances in therapy, it continues to put patients at risk of immune-compromising infections. ${ }^{9}$ These repeated infections and subsequent lower ANC levels may result in treatment delays and dose reductions ${ }^{3}$-as well as costly extended or repeated hospitalizations and eventually decreased survival. ${ }^{10,11}$

CIN's devastating impact on your patient's treatment regimen

CIN can not only compromise your patient's immune system and make them vulnerable to long-term infection, but it may also wreak havoc on the regimen you prescribed, making them face the devastating "4 Ds":

\section{DECREASE}

their recommended treatment dose

\section{DELAY}

their treatment cycles

\section{DOWNGRADE}

their prescribed regimen

\section{DISCONTINUE}

their recommended treatment dose 


\section{G-CSFs leave patients vulnerable}

- It takes up to 8 days for G-CSFs to accelerate the maturation and proliferation of neutrophil precursors in bone marrow ${ }^{1}$

- G-CSF benefits are not seen until the eighth day following chemotherapy ${ }^{12}$

- Patients treated with various chemotherapies may still experience Grade 4 neutropenia between Days $6-8$, even with the use of G-CSF ${ }^{12}$

\section{Cycle 1 of chemotherapy may} put patients at risk

- In Cycle 1, the ANC nadir takes place between Days 7-1013

- CIN episodes are the highest in Cycle 1 in patients with breast cancer ${ }^{9}$

- Most initial neutropenic events and infection events occur in Cycle $1^{14}$

- The risk of initial hospitalization spikes by Day 10, particularly in high-risk patients with febrile neutropenia (FN) ${ }^{15}$ (Figure 2)

- Chemotherapy-related death rates have been observed in certain study populations to be as high as 13\%, with $37 \%$ of these deaths occurring during Cycle $1^{16}$

\section{Patients go unprotected as early as Week 1}

G-CSFs may leave patients vulnerable to CIN as early as Week 1 of Cycle 1.12,17 Should their ANC drop and approach 1.0 (or below), they run the risk of encountering life-threatening consequences, including infection, FN, hospitalization, and death. ${ }^{18}$

\section{FIGURE 2: CUMULATIVE PROBABILITY \\ OF INITIAL HOSPITALIZATION FOR FN BY RISK COMPOSITE SCORE ${ }^{15}$}

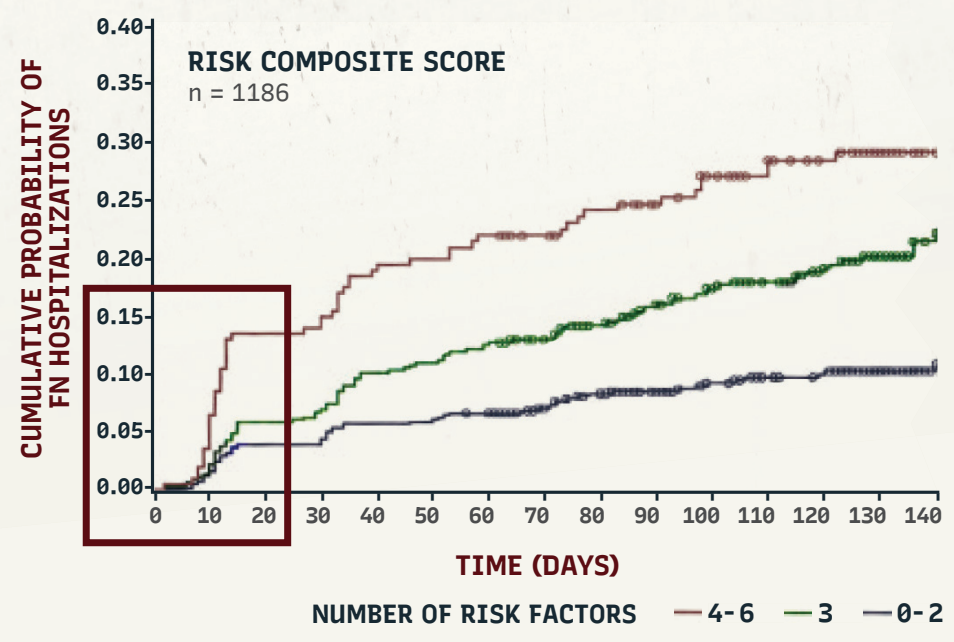

Lyman, G.H. and Delgado, D.J. (2003), Risk and timing of hospitalization for febrile neutropenia in patients receiving CHOP, CHOP-R, or CNOP chemotherapy for intermediate-grade non-Hodgkin lymphoma. Cancer, 98: 2402-2409.

https://doi.org/10.1002/cncr.11827 Copyright @2003 American Cancer Society

\section{THE RISK IS RERL}

Although infections may linger in the healthcare setting, it's critical to reduce exposure and protect patients with cancer during the COVID-19 pandemic. This is precisely why prophylactic prevention therapy is now being recommended to include patients who may also be at intermediate risk of developing FN. ${ }^{19}$

\section{LEARN MORE TODAY AT} CINRisk.com

References: 1. Jee J, Foote MB, Lumish M, et al. Chemotherapy and COVID-19 outcomes in patients with cancer. J Clin Oncol. 2020;38(30):35383546. 2. Kuderer NM, Choueiri TK, Shah DP, et al. Clinical impact of COVID-19 on patients with cancer (CCC19): a cohort study. Lancet. 2020;395(10241):1907-1918. 3. Lalami Y, Klastersky J. Impact of chemotherapy-induced neutropenia (CIN) and febrile neutropenia (FN) on cancer treatment outcomes: an overview about well-established and recently emerging clinical data. Crit Rev Oncol Hematol. 2017;120:163-179. 4. Crawford J, Dale DC, Lyman GH. Chemotherapy-induced neutropenia: risks, consequences, and new directions for its management. Cancer. 2004;100(2):228-237. 5. Crawford J. Prevention and treatment of chemotherapy-induced neutropenia. Clin Adv Hematol Oncol. 2013;11(8):514517. 6. Tai E, Guy GP, Dunbar A, Richardson LC. Cost of cancer-related neutropenia or fever hospitalizations, United States, 2012. J Oncol Pract. 2017;13(6):e552-e561. 7. Lyman GH. Management of chemotherapy-induced neutropenia with colony-stimulating factors. 2008;4(2):13-17. 8. Buckley SA, Othus M, Vainstein V, Abkowitz JL, Estey EH, Walter RB. Prediction of adverse events during intensive induction chemotherapy for acute myeloid leukemia or high-grade myelodysplastic syndromes. Am J Hematol. 2014;89(4):423-428. 9. Becker PS, Griffiths EA, Alwan LM, et al, NCCN guidelines insights: hematopoietic growth factors, version 1.2020. J Natl Compr Canc Netw. 2020;18(1):12-22. 10. Gupta A, Abbasi B, Gupta S. Management of chemotherapy induced neutropenia-an unmet clinical need. Am J Biomed Sci \& Res. 2019;4(5):313-318. 11. Halpern MT, Yabroff KR. Prevalence of outpatient cancer treatment in the United States: estimates from the Medical Panel Expenditures Survey (MEPS). Cancer Invest. 2008;26(6):647-651. 12. Burris HA, Belani CP, Kaufman PA, et al. Pegfilgrastim on the same day versus next day of chemotherapy in patients with breast cancer, non-small-cell lung cancer, ovarian cancer, and non-Hodgkin's lymphoma: results of four multicenter, double-blind, randomized phase II studies. J Oncol Pract. 2010;6(3):133-140. 13. Cheng C, Gallagher EM, Yeh JY, Earl MA. Rates of febrile neutropenia with pegfilgrastim on same day versus next day of CHOP with or without rituximab. Anticancer Drugs. 2014;25(8):964-969. 14. Culakova E, Thota R, Poniewierski MS, et al. Patterns of chemotherapy-associated toxicity and supportive care in US oncology practice: a nationwide prospective cohort study. Cancer Med. 2014;3(2):434-444. 15. Lyman GH, Delgado DJ. Risk and timing of hospitalization for febrile neutropenia in patients receiving CHOP, CHOP-R, or CNOP chemotherapy for intermediate-grade non-Hodgkin lymphoma. Cancer. 2003;98(11):2402-2409. 16. Gómez H, Hidalgo M, Casanova L, et al. Risk factors for treatment-related death in elderly patients with aggressive non-Hodgkin's lymphoma: results of a multivariate analysis. J Clin Oncol. 1998;16(6):2065-2069. 17. Li Y, Klippel Z, Shih X, Wang H, Reiner M, Page JH. Trajectory of absolute neutrophil counts in patients treated with pegfilgrastim on the day of chemotherapy versus the day after chemotherapy. Cancer Chemother Pharmacol. 2016;77(4):703-712.

18. Crawford J, Dale DC, Lyman GH. Chemotherapy-induced neutropenia: risks, consequences, and new directions for its management. Cancer. 2004;100(2):228-237. 19. Lyman GH, Kuderer NM. Personalized cancer supportive care in COVID-19 era. Ann Oncol. 2020;31(7):835-837. 


\section{Hand-Foot Skin Reaction Secondary to Sunitinib in a Patient With Metastatic Clear Cell Renal Cell Carcinoma}

Karen Férez-Blando, MD; Francisco J. Castro-Alonso, MD; Judith Dominguez-Cherit, MD; Maria T. Bourlon, MD, MSc

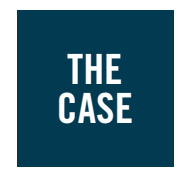

A man, age 45 years, was diagnosed with intermediate-risk stage IV clear cell renal carcinoma

(lung and lymph node metastases). He was prescribed first-line systemic treatment with sunitinib (Sutent) $50 \mathrm{mg}$ per day (each cycle: 4 weeks on, 2 weeks off). Upon day 22 of his second sunitinib cycle, he came to the oncology clinic complaining of difficulty walking due to bilateral sole pain. He described initial tingling sensations, which then became burning and painful, with symmetrical erythema and edema of the soles, without blisters.

These turned into painful plaques with yellowish discoloration and hyperkeratosis on pressure-bearing areas. $\mathrm{He}$ denied fever or other symptoms. The pain limited his instrumental activities of daily living, but not his self-care activities of daily living. Total body skin examination disclosed hyperkeratotic plaques on the undersurface of the great toes and heels of both feet, predominantly at sites of pressure (Figure 1); no blisters, crusts, ulcers, or fissures were found. No relevant findings were found upon physical examination of his hands, mucosae, and scalp. A diagnosis of grade 2 hand-foot skin reaction (HFSR) was made.
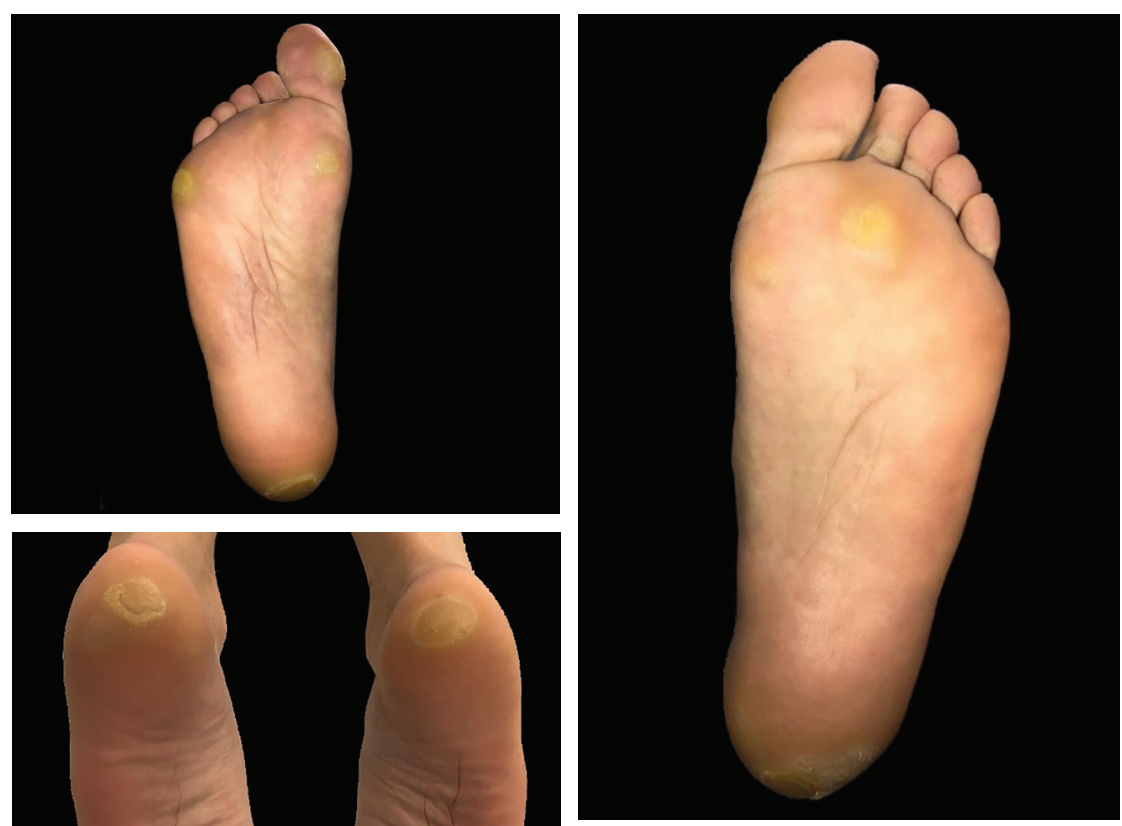

- Figure 1. Hyperkeratotic Plaques. These plaques, on the undersurface of the great toes and heels of both feet, occur predominantly at sites of pressure and friction.

\section{Besides oral analgesics, which of the following is the best treatment for this patient?}

A. Hot compresses and scrubbing the lesions

B. Low- or medium-potency topical steroid (ie, hydrocortisone $1 \%$ cream) plus a keratolytic agent (ie, urea 40\% cream)

C. High-potency topical steroid (ie,

clobetasol propionate $0.05 \%$ ointment) only
D. High-potency topical steroid (ie, clobetasol propionate $0.05 \%$ ointment) plus a keratolytic agent (ie, urea 40\% cream).

E. Discontinue sunitinib until improvement to grade 1 


\section{CORRECT ANSWER: D. High-potency topical steroid (ie, clobetasol propionate $0.05 \%$ ointment) plus a keratolytic agent (ie, urea $40 \%$ cream).}

Continued from page 272

\section{Discussion}

Hand-foot syndrome (HFS) is a welldescribed cutaneous adverse event (AE) of certain chemotherapeutic agents, like capecitabine, docetaxel, 5-fluorouracil, and pegylated liposomal doxorubicin. It was first described in 1974 by Zuelke; alternative names for this AE include acral erythema, palmar-plantar erythrodysesthesia, and Burgdorf reaction. ${ }^{1}$ Clinical dermatological manifestations may include erythema, edema, dryness, blisters, fissures, ulcers, and/or desquamation. Varying degrees of paresthesia, dysesthesia, and pain can accompany such changes. ${ }^{2}$ Although palms and soles are the most commonly affected areas, changes in the dorsal surfaces and nails can occur. ${ }^{3}$

With the advent of targeted therapies such as multikinase inhibitors (MKIs), a novel profile of cutaneous AEs has emerged. Since the MKI-related skin changes affecting the palms and soles differ from the ones caused by chemotherapy, the term HFSR is used when referring to the MKI-induced syndrome. ${ }^{4}$ The differences between HFS and HFSR are summarized in Table 1. HSFR has been associated with various MKIs, including regorafenib (Stivarga), cabozantinib (Cabometyx), sorafenib
(Nexavar), sunitinib, axitinib (Inlyta), and pazopanib (Votrient). ${ }^{5}$ Although it isn't life-threatening, HFSR can lead to cessation of therapy, dose reduction, and impaired quality of life. ${ }^{6}$

HFSR affects $31.2 \%$ to $79.4 \%$ of patients using MKIs. ${ }^{7-9}$ The frequency of all-grade HFSR differs greatly among the MKIs-for instance, the frequency with regorafenib is $46 \%$ to $84 \%$; with cabozantinib, $35 \%$; sorafenib, $10 \%$ to $62 \%$; and sunitinib, $10 \%$ to $28 \%$. The corresponding frequencies for grade 3 or greater HFSR are $20.4 \%, 9.5 \%, 2 \%$ to $36 \%$, and $4 \%$ to $12 \%$, respectively. Notably, the incidence of HFSR from a specific MKI can vary among tumor types, as different doses might be indicated for them. ${ }^{1,10,11}$

HFSR has several risk factors. In a meta-analysis of randomized controlled trials involving 24,956 patients, the

TABLE 1. Differences Between Hand-Foot Syndrome and Hand-Foot Skin Reaction

\begin{tabular}{|c|c|c|}
\hline & Hand-foot syndrome & Hand-foot skin reaction \\
\hline Causative agents & $\begin{array}{l}\text { Conventional chemotherapy (5-fluorouracil, cytarabine, } \\
\text { liposomal doxorubicin, etc) }\end{array}$ & MKIs (most), BRAF inhibitors (few), EGFR inhibitors (rare) \\
\hline Risk factors & Chemotherapy dosage; female gender & $\begin{array}{l}\text { MKI dosage; female gender; good performance status; tumor } \\
\text { type }\end{array}$ \\
\hline Clinical manifestations & $\begin{array}{l}\text { Diffuse erythema and edema, fissures, desquamation, and } \\
\text { blisters }\end{array}$ & $\begin{array}{l}\text { Focal, well-demarked yellow calluslike plaques with } \\
\text { erythematous rims; excessive desquamation in resolution phase }\end{array}$ \\
\hline Distribution & $\begin{array}{l}\text { Symmetric and diffuse; erythema over thenar and hypothenar } \\
\text { eminences, joints, and Achilles tendons; palms more involved } \\
\text { than soles }\end{array}$ & $\begin{array}{l}\text { Symmetric on pressure points and areas of increased friction; } \\
\text { soles more involved than palms }\end{array}$ \\
\hline Symptoms & $\begin{array}{l}\text { Dysesthesia begins as tingling sensations and progresses into } \\
\text { burning pain; decreases in pain and temperature sensation, } \\
\text { with preservation of light touch and proprioception }\end{array}$ & $\begin{array}{l}\text { Prodromal symptoms of numbness and tingling; paresthesia, } \\
\text { dysesthesia, pain, and decreased heat tolerance }\end{array}$ \\
\hline Hyperkeratosis & Rare & Often \\
\hline Onset & Weeks to months after starting treatment & Days to weeks after starting treatment \\
\hline Pathogenesis & $\begin{array}{l}\text { Accumulation of cytostatic agents in skin via eccrine sweat } \\
\text { ducts; damage to small nerve fibers }\end{array}$ & $\begin{array}{l}\text { Crosstalk between vascular endothelial cells and keratinocytes } \\
\text { through the release of s-HBEGF; possible local tissue damage } \\
\text { with insufficient repair due to inhibition of VEGFR and PDGFR }\end{array}$ \\
\hline Histopathology & Epidermal detachment and necrosis; damage to eccrine glands & $\begin{array}{l}\text { Epidermal hyperplasia; necrotic keratinocytes; interface } \\
\text { dermatitis }\end{array}$ \\
\hline Management & $\begin{array}{l}\text { Preventive: antiperspirant in palms and soles, regional cooling; } \\
\text { interruption or dose modification; high-potency topical } \\
\text { corticosteroids; wound care for ulceration and erosions; } \\
\text { topical keratolytic agents; frequent emollient use }\end{array}$ & $\begin{array}{l}\text { Preventive: avoidance of excessive pressure and repeated } \\
\text { friction; use of emollients, topical keratolytic agents, and topical } \\
\text { corticosteroids; dose escalation and transient dose modification } \\
\text { or drug withholding, according to severity grade }\end{array}$ \\
\hline Long-term sequelae & $\begin{array}{l}\text { Palmoplantar keratoderma; cases of patients losing their } \\
\text { fingertips; increased risk of secondary infections }\end{array}$ & None reported \\
\hline
\end{tabular}

MKI, multikinase inhibitor; PDGFR, platelet-derived growth factor receptor;

s-HBEGF, soluble heparin-binding epidermal growth factor. 
main risk factors for HFSR were:

(A) Tumor type. The highest risk was with thyroid cancer, with a relative risk (RR) of 12.62; the RR for renal cell carcinoma was 8.10 .

(B) MKI used. Cabozantinib represented the highest RR with 12.10; the RR with sunitinib was 2.97.

(C) Dosing. Severity and frequency of HFSR positively correlated with the dosage of MKI. ${ }^{12}$

(D) Additional risk factors. These include female sex, good performance status, preexisting calluses, poorly fitting shoes, and repeated friction to the hands and feet. ${ }^{5}$

The pathogenesis of HFSR is still unclear. Proposed mechanisms include direct cytotoxic effect of the MKIs to the epidermis, eccrine secretion of MKI, and MKI-induced deficient capillary maintenance in high-friction areas., ${ }^{9,13}$

A crosstalk between vascular endothelial cells and keratinocytes has also been suggested as an etiological mechanism. In this model, MKI triggers the release of soluble heparin-binding epidermal growth factor from vascular endothelial cells. This stabilizes sirtuin 1 , an essential keratinization inducer in keratinocytes, hence causing hyperkeratinization.

HFSR typically appears within the first 2 to 4 weeks of treatment initiation, with severity also likely to peak early.,14,15 HFSR clinical presentation can be divided into several phases. In the prodromal phase, the patient notices dysesthesia, which progresses over several days to burning pain; palms and soles become symmetrically erythematous and edematous. The inflammatory phase follows: A well-defined symmetrical erythema and tense bullae appear on the palms and soles. The hyperkeratotic phase is next: Localized welldemarcated painful yellowish hyperkeratotic patches overlie erythematous base pressure-bearing areas. ${ }^{5}$ The patient can experience paresthesia, dysesthesia, and pain, which may be

\section{TABLE 2. Patient Skincare Dos and Don'ts for HFSR Prevention}

\section{DO}

- Bathe with cold water

- Use mild soaps and syndets (synthetic detergents)

- Pat your skin to dry

- Apply moisturizing ointments several times a day

- Apply topical keratolytic agents (urea 10\%$40 \%$, ammonium lactate $6 \%-12 \%$, or salicylic acid 5\%-10\%) focally to treat hyperkeratosis

- Identify and eliminate sources of friction, such as ill-fitting clothing or shoes

- Wear appropriate shoes: When standing, there should be at least a half-inch of space for the longest toe at the end of each shoe, with room to wiggle all toes

- Sandals can be worn to relieve painful pressure points, if sun exposure is avoided

- Wear cotton socks and gloves at night to prevent injury and retain moisture

- Cool skin with packs as often as needed, but intermittently, not continuously

out of proportion to the clinical appearance of the lesions. Finally, the resolution phase is characterized by excessive desquamation and fissures. ${ }^{1,5,16,17}$ Other concomitant alterations have been described, including scalp dysesthesia, angular cheilitis, perianal rashes, and facial erythema resembling seborrheic dermatitis. It is uncertain whether the pathophysiology is the same. ${ }^{1}$

Several prophylactic measures have been described. It is recommended that before initiating treatment with a kinasetargeted therapy, a patient's hands and feet should be examined for existing hyperkeratotic lesions and, if present, debrided by a podiatrist or dermatologist. Patients should take measures to ensure that their skin remains extremely moisturized, utilizing $10 \%$ urea cream, and to avoid friction as much as possible (summarized in Table 2), and these measures should be maintained even if HFSR occurs. ${ }^{4,7,9,16}$ Answer $A$ is therefore incorrect: Hot compresses and scrubbing the lesions (no matter how byperkeratotic they look) will worsen HFSR.
Patients who use $10 \%$ urea cream prophylactically, 3 times a day, have lower rates of HFSR. This was observed in a randomized, open-label trial that included 871 patients with hepatocellular carcinoma being treated with sorafenib. The use of urea-based cream extended the time to the first occurrence of HFSR, and it improved patient quality of life compared with best supportive care. ${ }^{18}$ Other topical keratolytic agents (ammonium lactate 6\%-12\%; salicylic acid 5\%-10\%) can be used to treat focal hyperkeratosis. Higher concentrations of keratolytics (urea 40\%) might be implemented once HFSR is established. ${ }^{16}$ In addition, celecoxib has been suggested to lower incidence rates of HFSR. In an open-label prospective study of 116 patients with advanced hepatocellular cancer, patients who received sorafenib with celecoxib achieved lower grade 2 and grade 3 HFSR incidence rates compared with those who received sorafenib alone. ${ }^{19}$ Finally, a retrospective study found that prophylactic oral dexamethasone $2 \mathrm{mg}$, taken once a day, delayed dose modifications of 
regorafenib and decreased the incidence of grade 3 HFSR $3.2 \%$ in the experimental population vs $25.7 \%$ in the controls). ${ }^{20}$

After establishing a diagnosis of HFSR, treatment should be individualized using the National Cancer Institute's Common Terminology Criteria for Adverse Effects. HFSR of grade 1 is characterized by minimal changes, such as erythema, edema, or hyperkeratosis without pain, and it can be treated with topical lidocaine $2 \%$ to $6 \%$ in gel, cream, or patch form. In grade 2 HFSR, painful skin changes (eg, blisters, erythema, hyperkeratotic plaques) limit instrumental daily life activities, and grade $3 \mathrm{HFSR}$ is characterized by severe skin changes (eg, moist desquamation, ulceration, blistering, fissures, or local injury causing infectious complications) and pain that limits self-care activities of daily life., 56,21 Treatment of grade 2 and 3 HFSR involves pain control with systemic medications, including nonsteroidal anti-inflammatory drugs and opioids or $\gamma$-aminobutyric acid antagonists. ${ }^{7,17}$

Superpotent topical steroids, such as clobetasol propionate ointment $0.05 \%$ or fluocinonide $0.05 \%$, are recommended for treatment of the inflamed, erythematous areas. ${ }^{7,15}$ Topical steroids induce vasoconstriction, promote antimitotic actions, and have anti-inflammatory activity. ${ }^{22}$ No studies have compared the different strengths of topical steroids in HFSR. Superpotent ones are usually recommended since the percutaneous absorption in hyperkeratotic or lichenified lesions on the palms and soles is diminished. ${ }^{23,24}$ Clobetasol, due to its superior potency, has also proved effective in treating "steroid-resistant dermatosis." 25 The vehicle also matters; an ointment base, not cream, is preferred for infiltrated, lichenified lesions. An ointment's occlusive effect increases the hydration of the stratum corneum, enhancing the drug's penetration. ${ }^{26-28}$ Answer $B$ is therefore
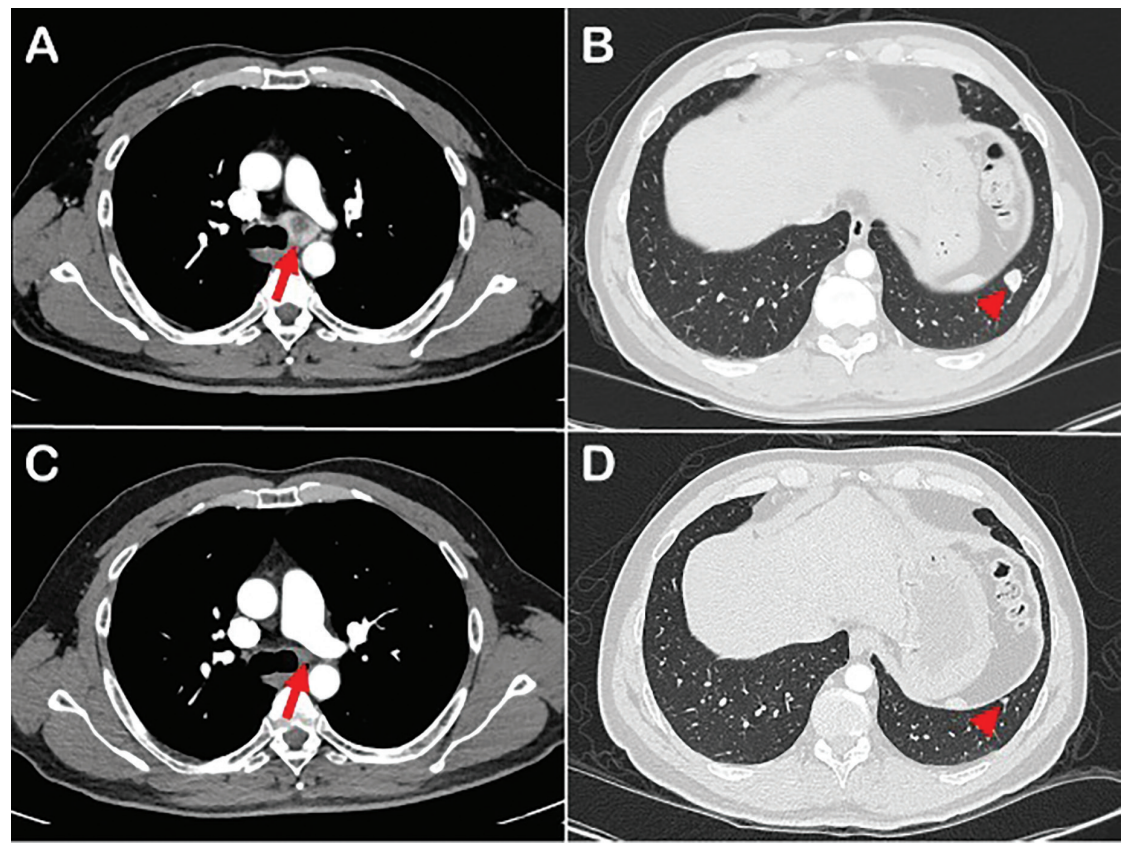

- Figure 2. Imaging Findings Pre- (superior) and Post (inferior) Sunitinib Treatment. Basal CT scan shows (A) an enlarged mediastinal lymph node (arrow) and (B) a pulmonary metastasis (arrowhead). Follow-up CT scan reveals partial response in (C) the mediastinal lymph node (arrow) and (D) pulmonary metastasis (arrowhead).

incorrect: Hydrocortisone $1 \%$ cream is a low-potency steroid. Steroids are an adjuvant to and not a substitute for the aforementioned supportive measures. Answer C is therefore incorrect: Superpotent steroids are not recommended as HFSR monotherapy.

Other translational studies have suggested treatment with sildenafil, heparin-containing ointments, ${ }^{29}$ and narrowband UV-B phototherapy, ${ }^{23}$ but more research is needed. ${ }^{15}$ In a randomized multicenter phase 2 trial, patients with metastatic renal cell carcinoma who were being treated with sorafenib found that hydrocolloid dressing containing ceramide improved symptom management of HFSR. ${ }^{24}$ An ongoing phase 3 randomized trial is investigating this therapy. ${ }^{30}$ Several other therapy trials with topical sildenafil (NCT03229512), tazarotene (NCT04071756), and nicotinamide (NCT04242927) are ongoing.

The option of holding MKI, as a measure to improve HFSR, should be reserved for severe cases (grade 3) or refractoriness to supportive measures. When holding is needed, most HFSR treatment algorithms recommend holding MKI for 7 days (or until HFSR symptoms resolve), then resuming at a lower dose. If HFSR worsens or does not improve, dose interruption or discontinuation is suggested. If severe HFSR recurs despite supportive measures, therapy should be discontinued. ${ }^{7}$ Answer $E$ is therefore incorrect: For grade 2 HFSR, try supportive measures before discontinuing the MKI. D, then, is the best answer.

Prognosis for patients with HFSR is good, since this $\mathrm{AE}$ is reversible and treatable. It has been suggested that HFSR occurrence is a predictor of prolonged survival. In individuals with renal cell carcinoma treated with sunitinib and sorafenib, HFSR is associated with significantly better progression-free survival (PFS). ${ }^{7,16}$ A similar observation was made in patients with 
metastatic colorectal cancer treated with regorafenib who experienced severe HFSR. ${ }^{31}$

\section{Outcome of This Case}

The patient was treated with a highpotency topical steroid (clobetasol $0.05 \%$ ointment twice daily) and a keratolytic agent (urea $40 \%$ cream once daily), which improved the symp- toms to grade less than or equal to 1 in 2 weeks. Clobetasol was tapered after 2 weeks. Rechallenge courses with urea were given when hyperkeratosis relapsed. Supportive care recommendations were reinforced. Sunitinib dose did not need to be delayed or adjusted. The patient had a partial response in his first CT response evaluation, which is maintained to this day (Figure 2). He has had a PFS of 12 months to date, with no grade greater than or equal to 2 HFSR recurrence.

FINANCIAL DISCLOSURE: The authors have no significant financial interest in or other relationship with the manufacturer of any product or provider of any service mentioned in this article.

For author bios and full reference list, visit cancernetwork.com/CQ_5.21
FROM THE PUBLISHERS OF:

Medical Economics

\section{Advanced Technology Will Help Drive Reimbursement Change}

\author{
Stephan Ondra, MD
}

L imited by the slow pace of change in traditional reimbursement models, shifts in the paradigm for patient care have also lagged far behind the possibilities created by transformative technology. The COVID-19 pandemic has changed that, catalyzing improvements in reimbursement by both commercial and government payers, not only for traditional telehealth but for other technologies that are advancing how we think about patient monitoring and care.

While the pandemic has accelerated the acceptance of telehealth and remote patient monitoring, the groundwork was being laid even prior to COVID-19. According to McKinsey \& Company, United States consumer telehealth adoption has skyrocketed from $11 \%$ in 2019 to $46 \%$ currently. Due to the needs created by the pandemic and the expansion of reimbursement for telehealth services, providers have rapidly scaled offerings and are seeing 50 to 175 times the number of patients via telehealth than they did before.

In addition to the explosion of telehealth usage by patients covered by both Medicare and commercial health plans, US policy makers have also embraced reimbursement for additional technologyenhanced methods to remotely monitor patients and identify those who could have care intervention prior to deterioration, and potentially prevent a full clinical deterioration. Such approaches not only provide a more convenient way for patients to be monitored, but also improve the efficiency of the system and the value of care delivery services by offering the information required to get the right level of care to the right patient at the right time.

As patients and providers become more comfortable with a combination of traditional approaches to care, telehealth, and remote patient monitoring technology, health care coverage will become more convenient and efficient, and of higher value. That's good news in the effort to lower the nation's health care costs while raising the level of patient care experiences and outcomes.

- Innovations drive care up, costs down. Telehealth alone can only begin to address the fundamental challenges of provider shortages and care access, and it changes the care paradigm in limited ways. Continuous remote patient monitoring (CRPM) is an important addition to traditional telehealth sessions for patients with chronic health issues. cRPM continuously monitors vital signs while the patient is at home and alerts the provider if health deterioration begins to occur-often before the patient feels any symptoms. The data shared give the provider the necessary information, early on, to determine the best treatment for the patient.

- Value-based reimbursement: the new norm. In order to continue to fund and advance cRPM and other artificial intelligence-based care delivery innovations, the reimbursement model must change in ways that enable and encourage the broader utilization of these innovations.

- Transformative models focus on prevention. Paradigm-shifting technology will be an important enabler in the transition to value-based reimbursement and related care models. The ultimate goal is to improve health outcomes and the care experience, while also saving money through more efficient targeting of care resources.

\section{Conclusions}

The future looks bright for further adoption of telehealth and the use of technologies such as cRPM. These and other innovations will become part of a portfolio of technology and other care model change-enablers destined to improve care outcomes while creating a more cost-effective solution to soaring health care costs and the finite resources that have been stretched even further due to the impact of COVID-19. As we move toward value-based reimbursement models, providers, insurers, and payers will need more proactive approaches that can measurably improve outcomes, save lives, and lower overall health care costs-often from the convenience of a patient's home.

To read the full article, visit https://bit.ly/3xaR7Zp 


\section{EXPERT COMMENTARY ON THE PRODUCT PROFILE OF Belantamab Mafodotin}

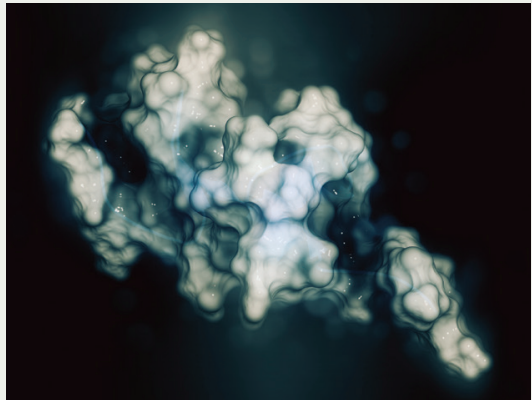

\section{Product Profile}

Drug name: Belantamab mafodotin-blmf (Blenrep)

Date of approval: August 5, 2020

Initial indication: Accelerated approval for the treatment of adult patients with relapsed or refractory multiple myeloma who have received at least 4 prior therapies including an anti-CD38 monoclonal antibody, a proteasome inhibitor, and an immunomodulatory agent ${ }^{1}$

Dosage and administration: $2.5 \mathrm{mg} / \mathrm{kg}$ administered as an intravenous infusion over approximately 30 minutes once every 3 weeks until disease progression or unacceptable toxicity

How supplied: $100 \mathrm{mg}$ as a lyophilized powder in a singledose vial for reconstitution and dilution

Pivotal clinical trial: Phase 2 DREAMM-2 trial (NCT03525678) 2

\section{Trial Design of DREAMM-2}

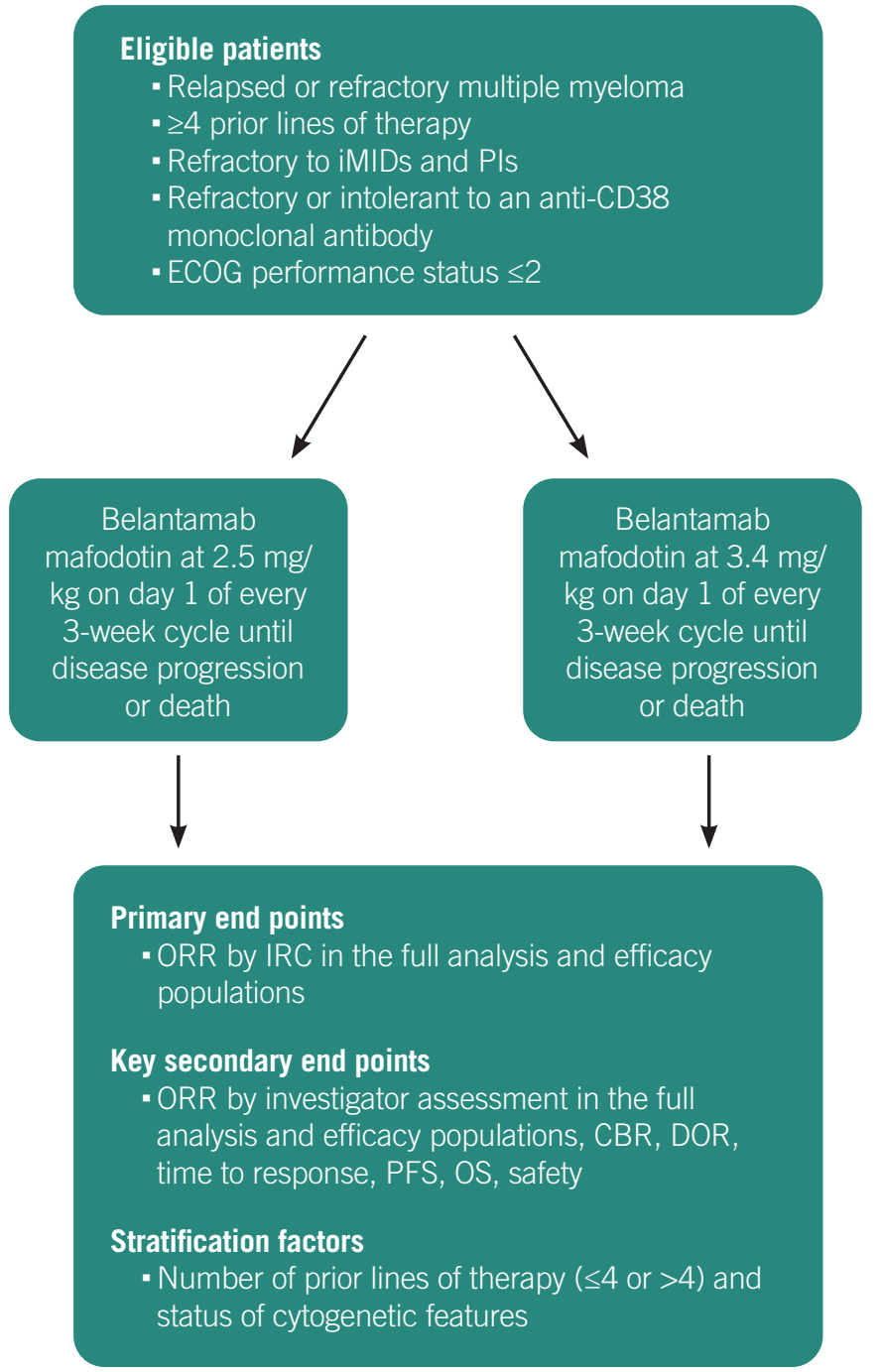

CBR, clinical benefit rate; DOR, duration of response; iMID, immunomodulatory drugs; IRC, independent review committee; ORR, overall response rate; OS, overall survival; PI, proteasome inhibitor; PFS, progression-free survival.

\section{REFERENCES}

1. FDA granted accelerated approval to belantamab mafodotin-blmf for multiple myeloma. FDA. Updated August 6, 2020. Accessed April 19, 2021. https://bit.ly/3dyY2ns

2. Lonial S, Lee HC, Badros A, et al. Belantamab mafodotin for relapsed or refractory multiple myeloma (DREAMM-2): a two-arm, randomised, open-label, phase 2 study. Lancet Oncol. 2020;21(2):207-221. doi:10.1016/S1470-2045(19)30788-0 


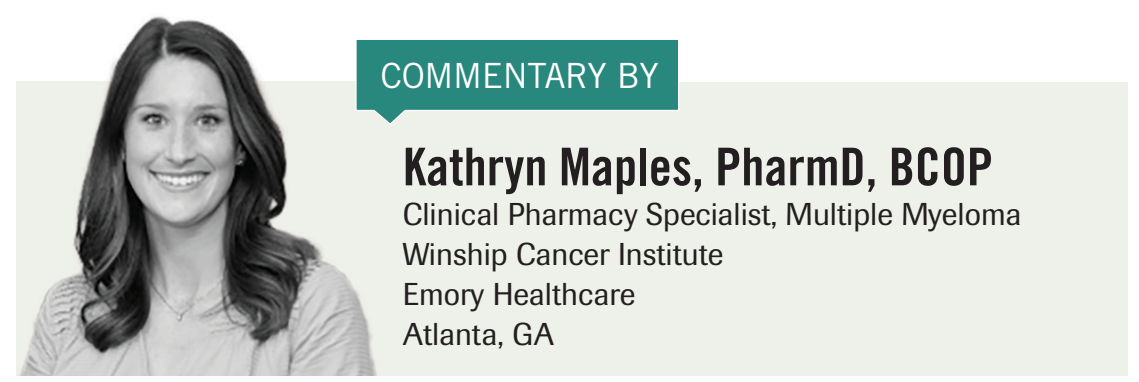

Q:

\section{Can you briefly describe}

the mechanism of action of belantamab mafodotin?

MAPLES: Belantamab mafodotin [belamaf] is a first-in-class, novel antibody-drug conjugate. It is an antibody targeting BCMA [B-cell maturation antigen], which is expressed on multiple myeloma cells. The antibody-drug conjugate has a payload of monomethyl auristatin F, which is more like our traditional cytotoxic chemotherapy [drugs]. The antibody component binds to the BCMA expressed on the myeloma cells. The whole antibody conjugate is taken into the cell, where the payload is then released to cause the cell death, so it helps deliver more of that cytotoxic chemotherapy into just those BCMAtargeted cells.

Q: What are some of the biggest concerns with the toxicity profile of bela-maf? Have any new safety concerns become more apparent with its use in the realworld treatment setting?

MAPLES: The top 3 grade 3/4 adverse effects that we saw in the clinical trials were keratopathy (changes to the cornea), thrombocytopenia, and anemia. Those are the top 3 adverse effects that we're seeing in clinical practice, as well. The most important thing to know, and especially [to discuss with] patients, is the [possibility of] keratopathy. Some patients will have symptomatic eye toxicity: blurry vision, dry eyes, or changes in their vision. Many patients will not have symptoms, but they'll have those cornea changes on exam. That's an important [distinction] to make to the patients, because they're not used to having their treatment held. Patients [with myeloma], especially at this stage in the game, have been on therapy for many, many years and are used to it. With bela-maf, we're having to dose, then hold, and dose, then hold, because of the keratopathy. [It's important to] explain to them that they may not have any symptoms, but what the eye doctor is seeing on their eyes is resulting in the dose being held. What we've seen in clinical practice has been consistent with what we saw on the clinical trials, and we are seeing that [with the use of] dose holds and dose reductions, these symptoms are resolving. And most commonly, I would say we're seeing them occur around cycle 2 or cycle 3 .

Beyond the eye toxicity, we most commonly see thrombocytopenia and anemia, especially in these heavily pretreated patients, so [management involves] utilizing platelet transfusions and blood transfusions, as needed.

\section{Q:}

\section{Do you recommend that all} patients who are on this drug be monitored by an eye care professional?

MAPLES: That's actually a requirement for the REMS [Risk Evaluation and Mitigation Strategy] program. In the clinical trials, the eye care professional had to be an ophthalmologist, but with the FDA approval, it can be any eye care professional. The patient will see this eye care professional for a baseline exam, prior to starting [bela-maf], and then they will see them every 3 weeks, prior to every single dose. It's a good thing to educate the patients on, because it's going to be 1 more doctor visit that they will have to have every 3 weeks, to make sure that we're following the REMS program and having their eyes checked. They will see those eye doctors very frequently, and we work very closely with them for any additional medications that they may need for their eye toxicity.

\section{Should patients and providers I. know about any additional aspects of the REMS program, beyond the close eye monitoring?} MAPLES: The REMS program has 3 key players. First, the providers have to enroll, and they will take an assessment quiz as part of their enrollment. But then they can assign delegates, so that could be their [physician assistant, nurse practitioner], or it could be their clinical pharmacists. I have been assigned as a delegate for my providers. Those are going to be the people who are assessing what the eye doctor tells us.

The eye doctor fills out a form that grades the patient's vision changes as well as their keratopathy changes. We input these data into the REMS program [to determine] if the patient can continue therapy. The patient also has to enroll, so we do that initially as part of their patient education.

The third component, which is important for infusion pharmacists and nurses to be aware of, is the infusion center, which also has to be enrolled in the REMS program. They have the final component, called the REMS checklist; once the drug is given, they sign off on the exact milligram dose that was given, and the day that it was given.

The eye doctors are not enrolled, but they're a key player. Making sure that you have all 3 of those things set up before you get your first patient going is very critical.

\section{Are dosing modifications 1 - common with this agent?}

MAPLES: If the patient experiences a grade 2 toxicity-be it visual acuity changes or keratopathy-the recommendation is to hold [the dose] until they return to grade 1 . At that point, you can resume 
at the $2.5-\mathrm{mg} / \mathrm{kg}$ dosing. However, if a patient experiences grade 3 toxicity, then the recommendation is to again hold until they return to grade 1 . At that point, you would reduce down to a $1.9-\mathrm{mg} / \mathrm{kg}$ dose. Based on of the grade of the toxicity, we're always going to be holding, but the difference between grade 2 and grade 3 is [that the latter] requires a dose reduction.

\section{Should clinicians be aware of $1 . \quad$ any major drug interactions?}

MAPLES: Luckily, with this medication, there are not many significant drug interactions. Of course, [we'd like] to avoid any other medications that cause eye toxicity. As this is an antibody-based medication, we don't have many significant drug interactions to worry about.

Q:

Have any barriers to the patient emerged since this agent's approval, such as common reimbursement issues or logistical challenges to obtaining the medication?

MAPLES: The biggest barrier is the REMS program. While it exists for a necessary reason and it's very doable, it is a cumbersome thing to get started, just to make sure that everything is in alignment. Our biggest barrier was making sure that we had everything set up at the infusion center before we could even get the drug delivered. If your institution, like mine, has multiple infusion sites, they may each have to be registered individually, so only 1 of ours is currently registered. Something for patients to keep in mind is that even if they're used to getting treatment at one place, they may have to get it somewhere else just based upon the requirements there.

In terms of reimbursement, the patients who we are putting on this medication have more than those 4 prior therapies, so just being mindful of the type of patient who you're initiating on this therapy will reduce any issues with getting reimbursement.
[You should] think about getting the eye appointment scheduled [for the patient]. This is not a therapy that you can decide on today and start treatment tomorrow. It will take at least a week or so to get set up, so that's something for the patient and the team to keep in mind.

FINANCIAL DISCLOSURE: The authors have no significant financial interest in or other relationship with the manufacturer of any product or provider of any service mentioned in this article.

\section{Submit your original clinical trial} manuscripts

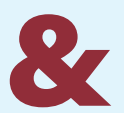

\author{
investigations \\ for publication.
}

\section{Submit Now}

\section{cancernetwork.com For more great content check out our website.}

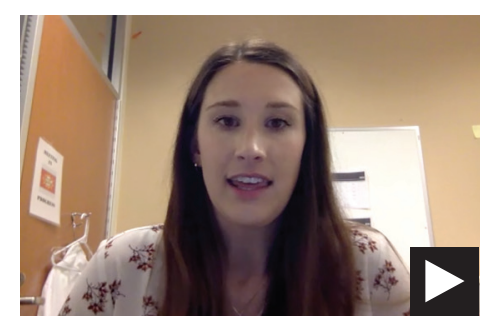

\section{Kathryn Maples, PharmD, BCOP, Discusses \\ Belantamab Mafodotin for Multiple Myeloma}

In an interview with ONCOLOGY ${ }^{\circledR}$, Kathryn Maples, PharmD, BCOP, offers a comprehensive review of real-world treatment considerations of belantamab mafodotin as therapy for adult patients with relapsed or refractory multiple myeloma who have received at least 4 prior therapies. She discusses toxicities associated with the agent, its mechanism of action, the REMs program, dosing modifications, and barriers to therapy administration.

...on the REMs Program for Belantamab Mafodotin

Cancernetwork.com/Maples_bela-maf1

...on Getting Centers Set Up for Belantamab Mafodotin Administration Cancernetwork.com/Maples_bela-maf2 

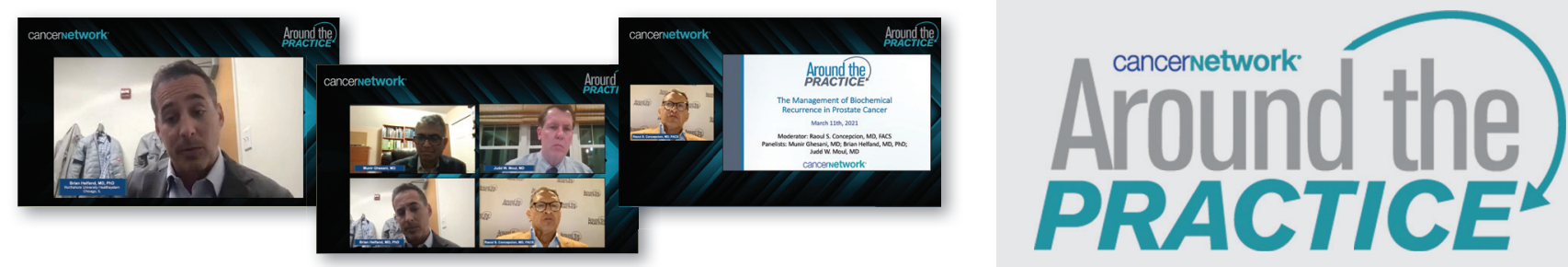

\section{MEET OUR}

EXPERT PANEL

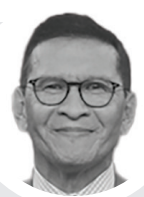

Raoul Concepcion, MD Comprehensive Prostate Center Nashville, TN

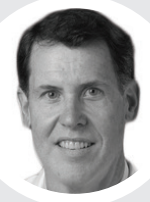

Judd Moul, MD

Duke Cancer Center Durham, NC

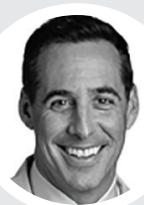

Brian Helfand, MD NorthShore University Health System \& University of Chicago Chicago, IL

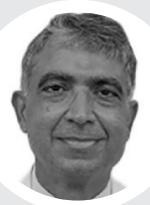

Munir Ghesani, MD Icahn School of Medicine at Mount Sinai New York, NY

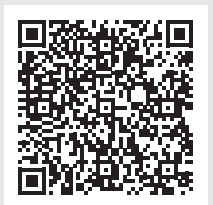

To watch the full event, scan the QR code

\section{The Management of Biochemical Recurrence in Prostate Cancer}

\section{KEY TAKEAWAYS:}

- After radical prostatectomy, a rise in prostate-specific antigen (PSA) level to $0.2 \mathrm{ng} / \mathrm{mL}$ or greater is considered recurrence. For patients with high-risk disease, consecutive rises in PSA level prior to reaching $0.2 \mathrm{ng} / \mathrm{mL}$ is also considered a recurrence.

- It is important to look at the surgical pathology because in some cases, benign glands at the margin can result in low levels of PSA that are not cancer related. Identifying recurrence is more challenging after definitive radiotherapy because the prostate gland remains in situ, and therefore some level of PSA is detectable.

- Therapy should be initiated for patients with Gleason scores of greater than 8 at the time of surgery, if biochemical recurrence is less than 3 years and if the PSA doubling time is less than 9 to 12 months.

- The recommended imaging modality is a positron emission tomography (PET) CT or PET MRI. The most widely available agent is fluciclovine, a fluorine 18-based agent, used to localize recurrent disease in patients who are presenting with increased PSA levels.

- There are multiple agents and combinations currently under investigation that may become available over the next few years. ${ }^{18} \mathrm{~F}$-DCFPyL, a second-generation prostate-specific membrane antigen PET agent, is furthest along in the FDA review process and will most likely be considered as the next agent for approval.

- Overall, the greatest unmet need for patients with prostate cancer is the ability to localize disease reliably with current imaging modalities at very low PSA levels.

$\mathrm{N}$ ew imaging technologies improve early detection of biochemical recurrence (BCR) after definitive treatment for prostate cancer, but the clinical role of these imaging modalities and management based on these results continue to have unanswered questions, according to experts who participated in a recent CancerNetwork ${ }^{\circledR}$ Around the Practice virtual presentation, moderated by Raoul Concepcion, MD, of the Comprehensive Prostate Center in Nashville, Tennessee. The presentation featured an interactive online platform in which audience members answered several polling questions, which were discussed by the panelists.

\section{Follow-up After Definitive Treatment for Prostate Cancer}

The first polling question asked the audience to estimate the percentage of patients definitively treated for prostate cancer who would subsequently have BCR. Of the respondents, $40 \%$ estimated a BCR rate of $30 \%$ to $50 \%$ in this group of patients, whereas $20 \%$ estimated rates of less than $10 \%, 10 \%$ to $30 \%$, and more than $50 \%$, respectively. The second polling question asked for a true or false response to the following statement: "For patients who do have a BCR, initial therapy should always be androgen deprivation therapy (ADT)." Of the respondents, $60 \%$ answered "false," and 40\% answered "true." 


\section{Poll results: For patients who do have a BCR, initial therapy should always be ADT.}

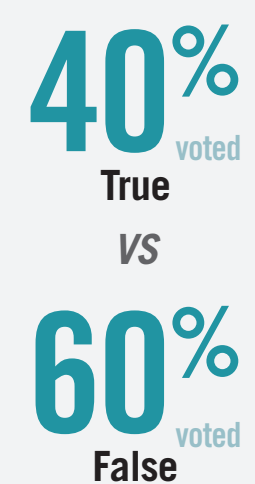

$A D T$, androgen deprivation therapy; $B C R$, biochemical recurrence.

For patients who received initial definitive therapy, the National Comprehensive Cancer Network (NCCN) guidelines recommend measuring prostate-specific antigen (PSA) every 6 to 12 months for 5 years and then annually thereafter, along with an annual digital rectal examination (which may be omitted if PSA is undetectable). ${ }^{1}$ Judd IMoul, MD, of the Duke Cancer Center in Durham, North Carolina, said that after radical prostatectomy, he typically sees his patients every 3 months for the first year. If their features are particularly highrisk, he will continue to see them every 3 to 4 months for up to 3 years, and if lower risk, he will decrease visits to every 6 months for the next 2 to 3 years and annually thereafter up to 10 years.

Although various PSA cutpoints have been used to define BCR, Brian Helfand, MD, of NorthShore University Health System and University of Chicago in Chicago, Illinois, said that he uses a PSA level of $0.2 \mathrm{ng} / \mathrm{mL}$ as the cutoff following radical prostatectomy, as results from one study showed that most $(86 \%)$ postoperative patients with PSA level greater than $0.2 \mathrm{ng} / \mathrm{mL}$ developed PSA progression by year 1 , with a $100 \%(95 \% \mathrm{CI}, 87 \%$ $100 \%$ ) risk of progression by year $3 .^{2}$ Helfand added that most people would

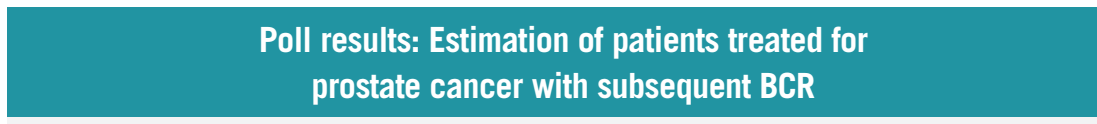

\section{What is your impression of the estimate of patients who have been definitively treated for prostate cancer who will subsequently have BCR?}

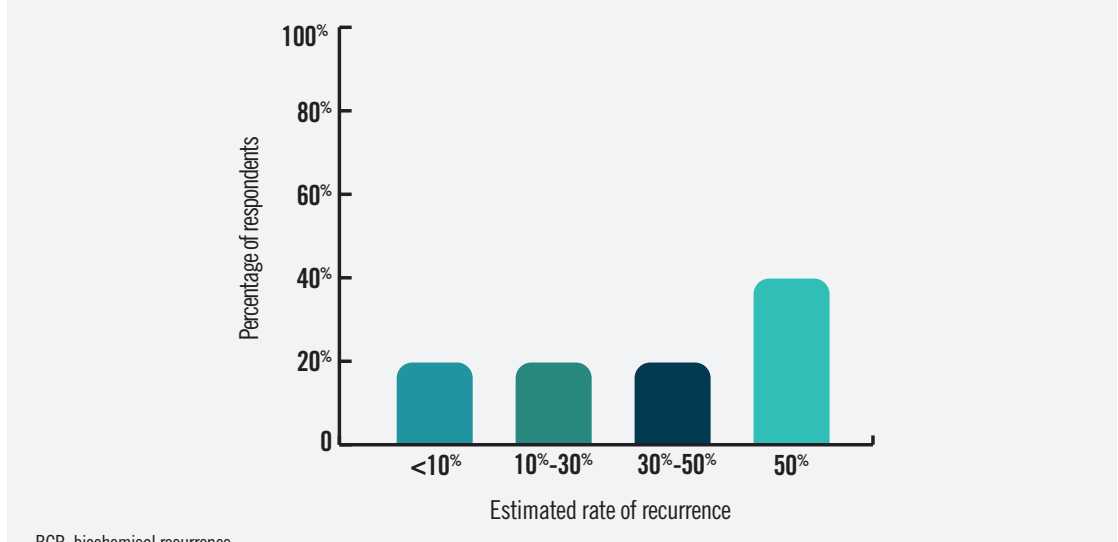

agree that consecutive rises in PSA on an ultrasensitive assay before reaching $0.2 \mathrm{ng} / \mathrm{mL}$ are also considered a BCR, especially with higher-risk disease.

Identifying recurrence following radiation therapy is more challenging because the in situ prostate gland produces a low level of PSA, according to Helfand. He uses what he refers to as the Phoenix definition (PSA level $2 \mathrm{ng} / \mathrm{mL}$ greater than the lowest PSA level reached), which had a sensitivity and specificity of $64 \%$ and $74 \%$, respectively, for predicting clinical failure in one study. ${ }^{3} \mathrm{He}$ added that consecutive rises in PSA level taken at least 1 month apart also increase suspicion for recurrence.

Although the PSA nadir usually occurs within 3 to 6 months following define $\mathrm{ADT}$ (androgen deprivation therapy) plus radiation therapy, Moul said that it can take up to 18 months to reach the PSA nadir following radiation therapy without ADT. Moul added that detectable PSA unrelated to cancer may be observed in a subset of patients who have benign glands at the surgical margin after radical prostatectomy.

When deciding whether to initiate treatment with a BCR, the panelists said that the timing of recurrence is important to consider, and Helfand added that a rapid rate of PSA doubling is particularly concerning.
Results from a study of 379 men with BCR showed that a pathological Gleason score of 8 or higher, 3 or fewer years from radical prostatectomy to recurrence, and PSA doubling time less than 3 months or 3.0 to 8.9 months were independently associated with shorter time to disease-specific mortality. Concepcion said that a patient with these risk factors would be at risk for succumbing to his disease if therapy were not initiated. ${ }^{4}$

Case 1: The panelists discussed the case of a White man, age 58 years, who presented in 2010 with a PSA level of $15 \mathrm{ng} / \mathrm{mL}$. Transrectal ultrasonography-guided biopsy showed that 5 of 12 core biopsies were positive and classified as Gleason group 2, and staging work-up was negative. Surgical pathology after robotically assisted radical prostatectomy showed pathologic stage of T3a. The PSA level was $0.8 \mathrm{ng} / \mathrm{mL} 6$ months after surgery, but follow-up was not done for another 3 years when he had a PSA level of $8.3 \mathrm{ng} / \mathrm{mL}$. The patient was asymptomatic and had no signs of metastatic disease on bone scan and CT. 


\section{CASE 1: If this patient presented today, and assuming you would restage the patient, what imaging modality would you choose?}

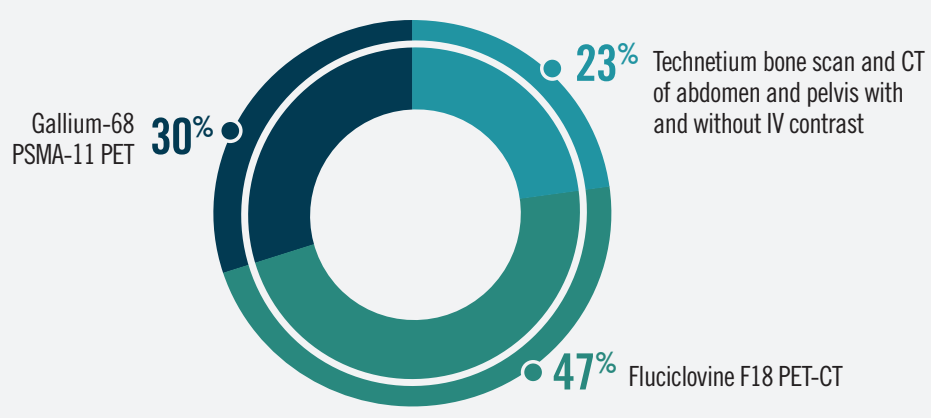

IV, intravenous; PET, positron emission tomography; PSMA, prostate-specific membrane antigen.

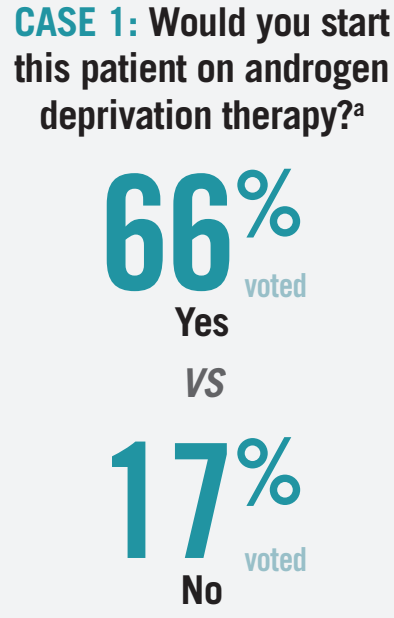

a Sixteen percent thought that not enough information was provided to make a decision.
The first polling question regarding case 1 asked the audience whether they would start this patient on ADT. Of the respondents, $66 \%$ answered yes, $17 \%$ answered no, and $16 \%$ said there was not enough information to decide.

Munir Ghesani, MD, of the Icahn School of Medicine at Mount Sinai in New York, New York, pointed out that the current standard of care for work-up has changed since 2013 and includes several imaging options beyond the conventional bone scan and CT (summarized in the NCCN guidelines). ${ }^{1}$ ${ }^{18} \mathrm{~F}$-fluciclovine is a positron emission tomography (PET)-CT radiotracer that, according to Ghesani, is currently available at most commercial radiopharmacies across the United States. The LOCATE trial (NCT02680041) showed that ${ }^{18} \mathrm{~F}$-fluciclovine PET-CT detected lesions in the pelvic region or prostate bed in $52 \%$ of patients with negative or equivocal pelvic CT and changed management plans in $59 \%$ of all patients. ${ }^{5}$ According to Ghesani, ${ }^{11} \mathrm{C}$-choline is also an approved radiotracer for PET, but its short half-life limits its availability to sites that have a cyclotron. In December 2020, the FDA approved Gallium-68 PSMA-11 $\left({ }^{68} \mathrm{Ga}-\mathrm{PSMA}-11\right)$ for PET for men with suspected metastases of prostate cancer that are potentially curable by surgery or radiation therapy or suspected recurrence of prostate cancer based on PSA levels, although Ghesani noted that the approval is currently limited to University of California, Los Angeles, University of California, San Francisco, and sites performing studies that have approached the FDA about cross-referencing the investigational new drug. ${ }^{6}$

A poll of the audience revealed that $47 \%$ of respondents would use ${ }^{18} \mathrm{~F}$-fluciclovine PET-CT to image and restage the case patient, whereas $30 \%$ would use ${ }^{68} \mathrm{Ga}$-PSMA-11 PET-CT and $23 \%$ would use technetium bone scan. However, the panelists noted that many insurance carriers cover only the ${ }^{18} \mathrm{~F}$-fluciclovine PET-CT after a bone scan and CT have been performed, and some even limit coverage to patients with a minimum PSA level of $2.0 \mathrm{ng} / \mathrm{mL}$. Furthermore, Ghesani said that bone scan and CT have low sensitivity for detection of BCR and often fail to show the extent of disease when recurrence is detected, which can lead to suboptimal targeting of treatment. He stressed the importance of collaborating with insurance providers and societies to eliminate the requirements for bone scans and CT prior to more sensitive imaging modalities in the near future.
Case 2: The panelists discussed the case of an African American man, age 64 years, who presented in March 2016 with a PSA level of $5.8 \mathrm{ng} / \mathrm{mL}$. Multiparametric MRI of the prostate revealed a prostate gland volume of $66.3 \mathrm{~mL}$ and a Prostate Imaging Reporting and Data System 4 lesion of $1.5 \mathrm{~cm}$ to the right peripheral zone. Mechanical fusion biopsy showed that 2 of 3 core biopsies of the right peripheral zone had Gleason group $3(4+3)$, and 3 of 12 systematic biopsies showed Gleason group $4(4+4)$ in the mid-left peripheral zone. The patient underwent external beam radiation therapy but declined ADT. Serial PSAs showed a rise from $0.8 \mathrm{ng} / \mathrm{mL}$ in June 2017 to $1.7 \mathrm{ng} / \mathrm{mL}$ in June 2018.

In the first polling question about the case, the majority (approximately 90\%) of the audience members recommended restaging the patient. In the next question, $56 \%$ of respondents said that they would use CT and technetium bone scan to restage the patient. Other answer choices receiving responses included ${ }^{18} \mathrm{~F}$-fluciclovine PET-CT 


\section{CASE 2: What imaging modality would you use to restage this patient?}

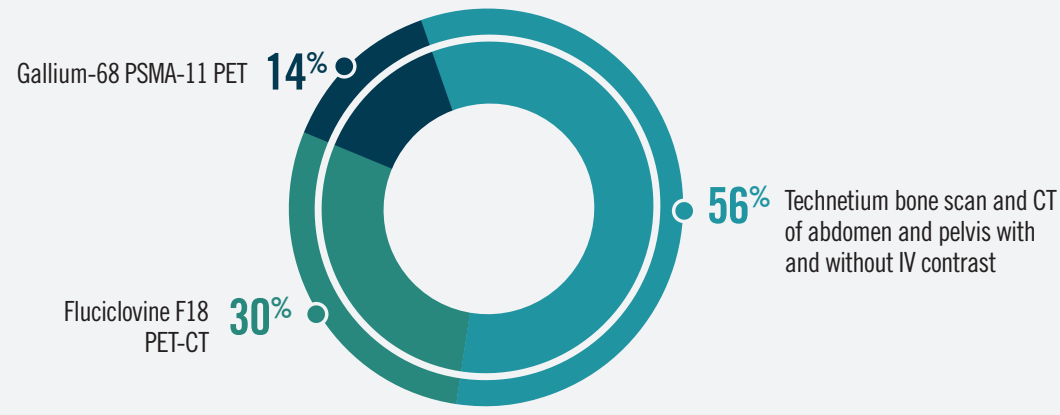

IV, intravenous; PET, positron emission tomography; PSMA, prostate-specific membrane antigen.

(30\%) and ${ }^{68}$ Ga-PSMA-11 PET-CT (14\%). Moul said that his approach to the case patient would depend in part on the patient's current health and life expectancy. "If he's in poor health, I would probably observe him," he said. "If he was in robust health, or...had a greater than 10-year life expectancy, I would work him up.” Moul added that ${ }^{18} \mathrm{~F}$-fluciclovine PET-CT would be "an ideal imaging test" in the work-up.

Ghesani said that according to at least 1 study, ${ }^{68}$ Ga-PSMA-11 PET-CT may have better sensitivity for BCR than ${ }^{18}$ F-fluciclovine PET-CT in the setting of a very low PSA level. Findings from a prospective trial (NCT03515577) showed that the detection rates were significantly higher with ${ }^{68} \mathrm{Ga}-\mathrm{PSMA}-11$ (56\%) than with ${ }^{18} \mathrm{~F}$-fluciclovine $(26 \%)$ in 50 patients with PSA level of 0.2 to $2.0 \mathrm{ng} / \mathrm{mL}$ (odds radio, $4.8 ; P=.0026) .{ }^{7}$ However, Ghesani said that ${ }^{18} \mathrm{~F}$-fluciclovine PET is still considerably more sensitive than CT and bone scan. "If only fluciclovine PET is available, it still will be very appropriate to do that scan," he said.

Ghesani added that patients are usually scanned sooner after injection with the ${ }^{18}$ F-fluciclovine PET-CT than with ${ }^{68} \mathrm{Ga}-\mathrm{PSMA}-11$, and the lower degree of urinary excretion may be advantageous for identifying small areas of recurrence in the prostate bed. Results of a prospective study of patients with BCR after definitive primary therapy $(\mathrm{N}=58)$ showed that detection rates of ${ }^{18} \mathrm{~F}$-fluciclovine versus ${ }^{68} \mathrm{Ga}$-PSMA-11 varied by location of recurrence: ${ }^{18} \mathrm{~F}$-fluciclovine had a higher rate of detection than that of ${ }^{68} \mathrm{Ga}-\mathrm{PSMA}-11$ for local recurrence $(37.9 \%$ vs $27.6 \% ; P=.03$ ), a similar rate for local pelvic lymph nodes (46.6\% vs $50 \%$; $P=.71$ ), and a lower rate for extrapelvic lymph nodes $(41.4 \%$ vs $51.7 \% ; P=.26)$ and bone $(25.9 \%$ vs $36.2 \%$; $P=.23) .^{8}$ The study authors concluded that further research is needed but suggested that the varying strengths of each tracer may complement each other. ${ }^{8}$

\section{Emerging Imaging Modalities and Future Directions}

Ghesani said that at least 5 combinations of 2 gallium-based and 3 fluorine-based radiotracer agents are in the pipeline, with the second-generation PSMA-PET agent ${ }^{18} \mathrm{~F}$-DCFPyL the furthest along in the FDA review process. However, Helfand noted that the ability to reliably localize disease with low PSA level remains the greatest unmet need regarding BCR and detection, and identifying the roles for recently approved and emerging radiotracers will be important moving forward. Moul concluded that although the new imaging modalities are clinically useful, care must be taken to avoid overly aggressive management, particularly in older patients. "Don't forget to keep your doctor's hat on and look at the patient, look what's in front of you, and put it in context," he said.

\section{CASE 2: Based on these PSA kinetics, would you restage the patient at this point?}

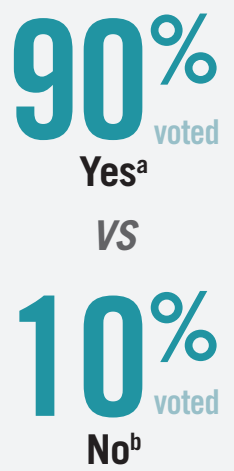

PSA, prostate-specific antigen.

aApproximately $90 \%$ reported "yes."

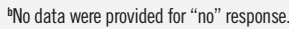

\section{REFERENCES}

1. NCCN. Clinical Practice Guidelines in Oncology. Prostate cancer, version 2.2021. Accessed April 2, 2021. https:// www.nccn.org/professionals/physician_gls/pdf/prostate.pdf 2. Freedland SJ, Sutter ME, Dorey F, Aronson WJ. Defining the ideal cutpoint for determining PSA recurrence after radical prostatectomy. prostate-specific antigen. Urology. 2003;61(2):365-369. doi:10.1016/s0090-4295(02)02268-9

3. Horwitz EM, Thames HD, Kuban DA, et al. Definitions of biochemical failure that best predict clinical failure in patients with prostate cancer treated with external beam radiation alone: a multi-institutional pooled analysis. J Urol. 2005;173(3):797-802. doi:10.1097/01. ju.0000152556.53602.64

4. Freedland SJ, Humphreys EB, Mangold LA, et al Risk of prostate cancer-specific mortality following biochemical recurrence after radical prostatectomy. JAMA. 2005;294(4):433-439. doi:10.1001/jama.294.4.433

5. Andriole GL, Kostakoglu L, Chau A, et al. The impact of positron emission tomography with 18f-fluciclovine on the treatment of biochemical recurrence of prostate cancer: results from the LOCATE trial. J Urol. 2019;201(2):322-331. doi:10.1016/j.juro.2018.08.050

6. $68 \mathrm{Ga}-\mathrm{PSMA}-11$. Prescribing information. University of California; 2020. Accessed April 7, 2021. https:// www.accessdata.fda.gov/drugsatfda_docs/ label/2020/212642s000lbl.pdf

7. Calais J, Ceci F, Eiber M, et al. 18F-fluciclovine PETCT and 68Ga-PSMA-11 PET-CT in patients with early biochemical recurrence after prostatectomy: a prospective, single-centre, single-arm, comparative imaging trial. Lancet Oncol. 2019;20(9):1286-1294. doi:10.1016/S14702045(19)30415-2

8. Pernthaler B, Kulnik R, Gstettner C, Salamon S, Aigner RM, Kvaternik H. A prospective head-to-head comparison of 18F-fluciclovine with 68Ga-PSMA-11 in biochemical recurrence of prostate cancer in PET/CT. Clin Nucl Med. 2019;44(10):e566-e573. doi:10.1097/ RLU.0000000000002703

Editor's Note: Interview quotes were slightly modified for readability. 


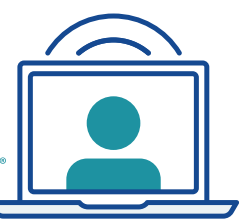

\section{Targeted Therapy Treatment Options for Patients With Relapsed/Refractory Multiple Myeloma}

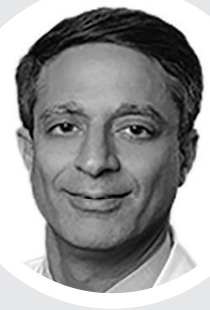

\section{Expert Interview With Sagar Lonial, MD}

Lonial discussed challenges and options in the management of relapsed/refractory multiple myeloma in later lines of therapy.

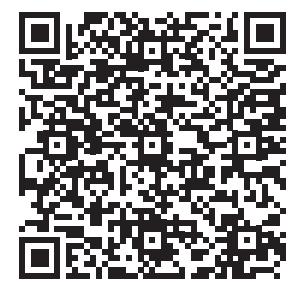

To see the full video with Lonial, scan the code.

\section{KEY TAKEAWAYS:}

- Relapse and disease progression are inevitable for patients with multiple myeloma (MM) and ultimately lead to the development of relapsed/refractory MM (RRMM).

- In patients with RRMM, each successive relapse has a less favorable treatment response and treatment options become more limited.

- Agents with novel mechanisms of action can offer potential options for patients with RRMM.

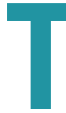
he second most common hematological malignancy in high-income countries, ${ }^{1}$ multiple myeloma (MM) accounts for $1 \%$ of neoplastic diseases ${ }^{1}$ and represents $18 \%$ of hematologic malignancies in the United States. ${ }^{2}$ More than 32,000 people received an MM diagnosis in the United States in $2020 .^{3}$ Although advances in treatment over the past 2 decades have enabled lengthier survival, the disease remains incurable $e^{4,5}$; the 5 -year survival rate is $53.9 \% .^{6}$

Relapse and disease progression are inevitable for patients with MM. Multiple relapses enable resistant clones to develop, meaning shorter consecutive relapses and the ultimate development of relapsed/ refractory MM (RRMM), characterized by muted response to, or resistance to, standard therapies. ${ }^{7,8}$
Of all patients with RRMM, 61.3\% received novel therapy in 2014, compared with $8.7 \%$ in 2000 , and survival has become longer in recent years. ${ }^{9}$ However, at some point, most patients with RRMM will stop responding to standard doublet or triplet regimens. These are composed of a proteasome inhibitor (PI), such as bortezomib, carfilzomib, or ixazomib; an immunomodulatory drug (IMiD), such as lenalidomide or pomalidomide; and a monoclonal antibody (mAb), such as daratumumab or elotuzumab, together with autologous stem cell transplantation (in certain patients). ${ }^{2,10}$ For patients with triple-class refractory MM (previously treated with a PI, an IMiD, and a CD38-directed $\mathrm{mAb})$, cytotoxic therapies-such as the combination of dexamethasone, 
thalidomide, cisplatin, doxorubicin, cyclophosphamide, and etoposidecan be effective, with response rates of about $50 \%$. However, the toxic adverse effects (AEs) and short duration of progression-free survival (PFS) mean these therapies are best used as a bridge to other treatments. ${ }^{9,11}$

"When we get to that level of treatment, typically 3 or 4 prior lines of therapy, it becomes a lot more challenging to find new drugs and new targets, as well as to manage effects from previous therapy and generalized debilitation that may occur with subsequent treatment of myeloma," said Sagar Lonial, MD, who is the Anne and Bernard Gray Family Chair in Cancer and professor and chair, Department of Hematology and Medical Oncology, Emory University School of Medicine, and chief medical officer, Winship Cancer Institute of Emory University, both in Atlanta.

The goal at that point in treatment, said Lonial, is to stabilize the disease. "What we want to do is improve quality of life," he explained. "We want the treatment to be tolerated well enough that patients can continue in the context of stable disease, and that some of the effects of progression-whether anemia, or renal insufficiency, or other issues-are addressed with the stabilization of disease."

\section{Approved Approaches to RRMM}

Selinexor, which inhibits the XPO1 protein and stimulates tumor-suppressor proteins, ${ }^{2,12}$ was approved by the FDA in December 2020 for use in combination with bortezomib and dexamethasone for treatment of patients with MM who have received at least 1 prior therapy. ${ }^{13}$ It was tested in the STORM trial in 122 participants with triple-class refractory MM. ${ }^{12}$

Patients in the trial had previous exposure to bortezomib, carfilzomib, lenalidomide, pomalidomide, daratumumab, and an alkylating agent, and their disease was refractory to at least $1 \mathrm{PI}, 1 \mathrm{IMiD}$, and daratumumab. All patients were given $80 \mathrm{mg}$ of oral selinexor plus 20 mg of dexamethasone twice a week. The primary end point was overall response rate (ORR), defined as confirmed partial response or better as assessed by an independent review committee; overall survival (OS) and response duration were assessed as secondary end points. ${ }^{12}$

The median duration of response was 4.4 months (95\% CI, 3.7-10.8) and the median PFS was 3.7 months (95\% CI, 3.0-5.3). A partial response or better (defined as $\geq 50 \%$ reduction in the serum level of myeloma protein) occurred in $26 \%$ of patients $195 \%$ CI, $19 \%-35 \%$ ) and included 2 stringent complete responses, 6 very good partial responses, and 24 partial responses. Thirty-nine percent of patients had a minimal response or better (defined as $\geq 25 \%$ to $<50 \%$ reduction in the serum level of myeloma protein; 95\% CI, $31 \%-49 \%$ ). Median OS was 8.6 months (95\% CI, 6.2-11.3); patients who had a minimal or partial response or better had a median OS of 15.6 months. ${ }^{12}$

Thrombocytopenia occurred in $73 \%$ of STORM study participants, while $73 \%, 72 \%$, and $67 \%$ experienced fatigue, nausea, and anemia, respectively. Grade 3 or higher AEs included thrombocytopenia $(59 \%)$, anemia $(44 \%)$, hyponatremia $(22 \%)$, and neutropenia $(21 \%)$. Treatment was discontinued by $18 \%$ of participants because of AEs determined to be related to the study drugs. ${ }^{12}$

Melphalan flufenamide (melflufen), an anticancer peptide-drug conjugate, was approved by the FDA in February 2021 for patients with RRMM following 4 or more prior lines of therapy. ${ }^{14}$ Melflufen was evaluated in the multicenter, single-arm HORIZON trial, which enrolled 157 patients with RRMM refractory to pomalidomide and/ or an anti-CD38 monoclonal antibody, of whom $119(76 \%)$ had triple-classrefractory disease, $55(35 \%)$ had extramedullary disease, and 92 (59\%) were refractory to previous alkylator therapy. ${ }^{15}$ The ORR (the primary end point) was $29 \%$ (95\% CI, $22 \%-37 \%$ ) in the all-treated population and $26 \%$ (95\% CI, 18\%-35\%) in those patients with triple-class-refractory disease. In the all-treated and triple-class-refractory populations, median PFS was 4.2 months (95\% CI, 3.4-4.9) and 3.9 months (95\% CI, 3.0-4.6), respectively. ${ }^{15}$

All 157 patients in the treated population reported treatment-emergent AEs, whereas 149 (95\%) reported at least 1 melflufen-related treatmentemergent AE. A total of 150 patients $(96 \%)$ experienced grade 3 or higher treatment-emergent AEs, most commonly neutropenia (124 [79\%]), thrombocytopenia (120[76\%]), and anemia (67 [43\%]). ${ }^{15}$

Another treatment option for heavily pretreated RRMM is an antibody-drug conjugate (ADC), which combines mAbs specific to surface antigens with cell-killing agents. An ideal ADC has a $\mathrm{mAb}$ that is highly selective for a tumor-associated antigen but has restricted or no expression on healthy cells, a potent cytotoxic agent designed to induce target-cell death, and a linker that is stable in circulation but releases the cytotoxic agent in target cells. ${ }^{16}$

The first B-cell maturation antigen (BCMA)-targeted ADC to be approved for MM, belantamab mafodotin (belamaf) was approved by the FDA in August 2020 for use in patients with triple-class refractory MM previously treated with at least 4 medications. ${ }^{17}$

Belamaf was examined in the DREAMM-2 study, an international, multicenter, open-label phase 2 trial of patients with RRMM who had experienced disease progression after at least 3 lines of therapy and were refractory to both immunomodulatory drugs and proteasome inhibitors, and refractory and/or intolerant to an anti-CD38 monoclonal antibody, with an ECOG performance score of 0 to 2 . A total of 196 patients were randomized to receive either $2.5 \mathrm{mg} / \mathrm{kg}$ or $3.4 \mathrm{mg} / \mathrm{kg}$ of intravenous belamaf every 3 weeks 
until disease progression or unacceptable toxicity. Patients in the $2.5-\mathrm{mg} / \mathrm{kg}$ cohort had a median of 7 previous lines of therapy; patients in the 3.4-mg/kg cohort had a median of 6 previous lines of therapy. ${ }^{18}$

Thirty (31\%; $97.5 \%$ CI, 20.8\%-42.6\%) of 97 patients in the $2.5-\mathrm{mg} / \mathrm{kg}$ cohort and $34(34 \%$; $97.5 \%$ CI, $23.9 \%-46.0 \%)$ of 99 patients in the $3.4-\mathrm{mg} / \mathrm{kg}$ cohort had an overall response as assessed by an independent review committee. Eighteen patients $(19 \%)$ in the $2.5-\mathrm{mg} / \mathrm{kg}$ cohort, and 20 patients $(20 \%)$ in the $3.4-\mathrm{mg} / \mathrm{kg}$ cohort achieved a very good or better partial response. In total, 33 patients $(34 \%$; $95 \%$ CI, $24.7 \%$ $44.3 \%)$ in the $2.5-\mathrm{mg} / \mathrm{kg}$ cohort and 39 (39\%; 95\% CI, $29.7 \%-49.7 \%)$ in the $3.4-\mathrm{mg} / \mathrm{kg}$ cohort achieved a clinical benefit, defined as minimal response or better as assessed by the independent review committee. ${ }^{18}$

The chance of a duration of response lasting 4 months or longer was estimated to be $78 \%(95 \% \mathrm{CI}, 57 \%-89 \%)$ in the $2.5 \mathrm{-mg} / \mathrm{kg}$ cohort and $87 \%(95 \% \mathrm{CI}$, $69 \%-95 \%$ ) in the $3.4-\mathrm{mg} / \mathrm{kg}$ cohort. ${ }^{18}$

As of data cutoff on June 21, 2019, 18 patients in the $2.5-\mathrm{mg} / \mathrm{kg}$ cohort and 25 in the $3.4-\mathrm{mg} / \mathrm{kg}$ had a response duration of 4 months or longer; PFS follow-up was ongoing. The median PFS was 2.9 months (95\% CI, 2.1-3.7) in the $2.5-\mathrm{mg} / \mathrm{kg}$ cohort and 4.9 months (95\% CI, 2.3-6.2) in the $3.4-\mathrm{mg} / \mathrm{kg}$ cohort. At data cutoff, $56(58 \%)$ participants in the $2.5-\mathrm{mg} / \mathrm{kg}$ cohort and $55(56 \%)$ in the $3.4-\mathrm{mg} / \mathrm{kg}$ cohort had died or were experiencing disease progression. ${ }^{18}$

The most common grade 3 or 4 AEs were keratopathy (an anticipated AE associated with monomethyl auristatin $\mathrm{F}$, the payload in belamaf), ${ }^{19}$ experienced by 26 patients $(27 \%)$ in the $2.5-\mathrm{mg} / \mathrm{kg}$ cohort and 21 patients $(21 \%)$ in the $3.4-\mathrm{mg} / \mathrm{kg}$ cohort; thrombocytopenia, experienced by 19 patients $(20 \%)$ in the $2.5-\mathrm{mg} / \mathrm{kg}$ group and 33 patients $(33 \%)$ in the $3.4-\mathrm{mg} / \mathrm{kg}$ group; and anemia, experienced by 19 patients $(20 \%)$ in the $2.5-\mathrm{mg} / \mathrm{kg}$ group and 25 patients $(25 \%)$ in the $3.4-\mathrm{mg} / \mathrm{kg}$ group. ${ }^{18}$
Overall, 93 patients $(98 \%)$ in the $2.5-\mathrm{mg} / \mathrm{kg}$ cohort and 99 patients $(100 \%)$ in the $3.4-\mathrm{mg} / \mathrm{kg}$ cohort experienced AEs. AEs leading to dose reductions were reported in 28 patients $(29 \%)$ in the $2.5-\mathrm{mg} / \mathrm{kg}$ group and 41 patients $(41 \%)$ in the $3.4-\mathrm{mg} / \mathrm{kg}$ group. AEs leading to dose delays occurred in 51 patients $(54 \%)$ in the $2.5-\mathrm{mg} / \mathrm{kg}$ cohort and in 61 patients $(62 \%)$ in the $3.4-\mathrm{mg} / \mathrm{kg}$ cohort. AEs led to treatment discontinuation in 8 patients in the $2.5 \mathrm{-mg} / \mathrm{kg}$ cohort and 10 patients in the $3.4-\mathrm{mg} / \mathrm{kg}$ cohort. ${ }^{18}$

\section{The Challenge of Heavily Pretreated RRMM}

It is more difficult to treat heavily pretreated patients because they have already failed multiple lines of therapy. Lonial, an author on the DREAMM-2 study, is optimistic about the potential benefits of belamaf for these patients. "The ORR was about $30 \%$ and the median PFS was about 3 months, ${ }^{18}$ but the duration of response was close to 11 months," ${ }^{20}$ he said. "To me, that 11 months really speaks to both efficacy and tolerability of the treatment." He pointed out that although a majority of participants experienced keratopathy, the most common manifestations of this condition were dry or itchy eyes or minor changes in visual acuity. "Only $18 \%$ of patients who received belamaf actually had to have the drug held because of a significant change in visual acuity or [because of] corneal keratopathy," he said. ${ }^{18}$

DREAMM-6 (NCT03544281), an ongoing phase $1 / 2$ study of belamaf in combination with lenalidomide/ dexamethasone or bortezomib/dexamethasone, involves 18 patients with heavily pretreated RRMM refractory to an IMiD and to a PI, and refractory or intolerant to an anti-CD38 $\mathrm{mAb}$ in the DREAMM-2 study. In this trial, keratopathy occurred in all 18 patients and led to dose reductions in 7 (39\%) and dose delay in 15 (83\%), with no discontinuations. ${ }^{21}$
As a BCMA-targeted therapy, belamaf avoids the off-target AEs and potential cytotoxicity associated with treatments such as chemotherapy. "CD38, while it is clearly effective, is expressed on a number of different cells that may potentially result in some chronic long-term immunosuppression through the use of CD38-targeted therapy," explained Lonial. "Elotuzumab, which targets SLAMF7, also appears to have some activity, but there are some off-target effects in terms of NK [natural killer] cells. ${ }^{22}$ So the ability for that to be more focused on a myeloma cell is not as clear as it is with a target like BCMA, which is almost exclusively expressed on plasma cells."

This more narrow expression is a positive for patients with RRMM, explained Lonial. "We know that targeting BCMA as part of the normal response is part of what keeps a myeloma cell alive. It's part of what induces proliferation of a myeloma cell, and ligating a BCMA with its natural ligands also induces drug resistance," he said. "So being able to block those 3 important things that myeloma cells like to do, by targeting BCMA - even if it doesn't result in cell death-may resensitize cells, or slow down their growth, or slow down their drug resistance overall. So BCMA is in many ways almost an ideal target in myeloma."

Belamaf may not be right for every patient, and Lonial takes a practical approach when individualizing therapy. "One [should] consider some very specific patient-related issues," he said. "For instance, how far away does the patient live? How often do they want to come into the clinic? What is their degree of sensitivity to certain [adverse] effects or toxicity?"

In the case of belamaf, Lonial said, patients need to be seen at the clinic only every 3 weeks, but they also need regular examinations by an ophthalmologist because of the risk of keratopathy. A typical exam day would be that "they see [their ophthalmologist] in the morning, then us in the afternoon. If everything is $\mathrm{OK}$, 
we dose the patient in the afternoon. I think that represents a pretty significant supportive care approach that can reduce the incidence of significant keratopathy or AEs," noted Lonial.

With selinexor plus dexamethasone, patients must be willing to accept potential gastrointestinal upset in the form of nausea, diarrhea, and vomiting. According to Lonial, "[We need to] balance out the patient's expectations for treatment, benefits, and AEs, their distance from the clinic, and issues related to supportive care-those all weigh in as we think about how to approach a patient in the refractory myeloma setting."

\section{Future Treatment Options for RRMM}

Other BCMA-targeted therapies being considered for the treatment of RRMM include chimeric antigen receptor (CAR) T-cell therapy and bispecific antibodies. The advantage of CAR T-cell therapy, used for various kinds of cancer, is that patients are given their own genetically modified cells without any need for medication. ${ }^{5}$ Although response rates are generally high, Lonial pointed out that the time it takes to alter a patient's cells can be a hurdle.

"The challenge is that not every patient to whom you want to give a CAR T-cell therapy can wait the 6 weeks to have that product manufactured," Lonial said. He also pointed out that the likelihood of relapse is considerable, unlike the situations when CAR T-cell therapy is used against acute lymphoblastic lymphoma or diffuse large B-cell lymphoma.

Bispecific antibodies that target BCMA and another tumor-specific antigen such as CD19 or CD38 have been developed to improve clinical efficacy by reducing the risk of disease relapse due to antigen escape. The advantage of bispecific antibodies is that they can be administered immediately, but they can cause AEs such as cytokine release syndrome and neurotoxicity. ${ }^{23}$

Other treatments being studied include bispecific antibodies and CAR T cells that target the gene GPRC5D, and therapies that target cell surface antigen FcRH5. ${ }^{5}$ "While we are probably curing about $10 \%$ to $15 \%$ of [patients with] myeloma with what we have circa 2020, I think that BCMA, GPRC5D, FcRH5, and mutation-driven therapies have the real promise of taking that number to $60 \%$, $70 \%$, or even $80 \%$ down the road, and we just have to figure out how to do it," Lonial said.

\section{REFERENCES}

1. van de Donk NWCJ, Pawlyn C, Yong KL. Multiple myeloma. Lancet. 2021;397(10272):410-427. doi:10.1016/S0140-6736(21)00135-5

2. Kumar SK, Callander NS, Adekola K, et al. Multiple myeloma, version 3.2021, NCCN Clinical Practice Guidelines in Oncology. J Nat/ Compr Canc Netw. 2020;18(12):1685-1717. doi:10.6004/jnccn.2020.0057

3. Siegel RL, Miller KD, Jemal A. Cancer statistics, 2020. CA Cancer J Clin. 2020;70(1):7-30. doi:10.3322/caac. 21590

4. Kumar SK, Lee JH, Lahuerta JJ, et al; International Myeloma Working Group. Risk of progression and survival in multiple myeloma relapsing after therapy with IMiDs and bortezomib: a multicenter Internationa Myeloma Working Group study. Leukemia. 2012;26(1):149-157. doi:10.1038/leu.2011.196

5. Shah UA, Mailankody S. Emerging immunotherapies in multiple myeloma. BMJ. 2020;370:m3176. doi:10.1136/bmj.m3176

6. Cancer stat facts: myeloma. National Cancer Institute/Surveillance, Epidemiology, and End Results Program. Accessed March 5, 2021.

https://seer.cancer.gov/statfacts/html/mulmy.html

7. Chim CS, Kumar SK, Orlowski RZ, et al. Management of relapsed and refractory multiple myeloma: novel agents, antibodies, immunotherapies and beyond. Leukemia. 2018;32(2):252-262. doi:10.1038/leu.2017.329. Published correction appears in Leukemia. 2019;33(4):1058-1059.

8. Egan JB, Shi C-X, Tembe W, et al. Whole-genome sequencing of multiple myeloma from diagnosis to plasma cell leukemia reveals genomic initiating events, evolution, and clonal tides. Blood. 2012;120(5):10601066. doi:10.1182/blood-2012-01-405977

9. Mikhael J. Treatment options for triple-class refractory multiple myeloma. Clin Lymphoma Myeloma Leuk. 2020;20(1):1-7. doi:10.1016/j.clml.2019.09.621

10. Bazarbachi AH, Al Hamed R, Malard F, Harousseau J-L, Mohty M. Relapsed refractory multiple myeloma: a comprehensive overview. Leukemia. 2019;33(10):2343-2357. doi:10.1038/ s41375-019-0561-2

11. Toocheck C, Pinkhas D. Treatment of relapsed multiple myeloma complicated by cardiac extramedullary plasmacytoma with D-PACE chemotherapy. BMJ Case Rep. 2018;2018:bcr2017223611. doi:10.1136/bcr-2017223611

12. Chari A, Vogl DT, Gavriatopoulou M, et al. Oral selinexor-dexamethasone for triple-class refractory multiple myeloma. N Engl J Med. 2019;381(8):727738. doi:10.1056/NEJMoa1903455
13. FDA approves selinexor for refractory or relapsed multiple myeloma. FDA. December 18, 2020. Accessed March 5, 2021. https://www.fda.gov/drugs/drugapprovals-and-databases/fda-approves-selinexorrefractory-or-relapsed-multiple-myeloma

14. FDA grants accelerated approval to melphalan flufenamide for relapsed or refractory multiple myeloma. FDA. Updated March 1, 2021. Accessed April 13, 2021. https://www.fda.gov/drugs/drugapprovals-and-databases/fda-grants-acceleratedapproval-melphalan-flufenamide-relapsed-orrefractory-multiple-myeloma

15. Richardson PG, Oriol A, Larocca A, et al; HORIZON (OP-106) Investigators. Melflufen and dexamethasone in heavily pretreated relapsed and refractory multiple myeloma. J Clin Oncol. 2021;39(7):757-767. doi:10.1200/JCO.20.02259

16. ADC Review Editorial Team. What are antibodydrug conjugates? ADC Review. March 22, 2019. Accessed April 13, 2021. https://www.adcreview. com/the-review/antibody-drug-conjugates/what-areantibody-drug-conjugates

17. FDA granted accelerated approval to belantamab mafodotin-blmf for multiple myeloma. FDA. August 6 , 2020. Accessed March 5, 2021. https://www.fda.gov/ drugs/drug-approvals-and-databases/fda-grantedaccelerated-approval-belantamab-mafodotin-blmfmultiple-myeloma

18. Lonial S, Lee HC, Badros A, et al. Belantamab mafodotin for relapsed or refractory multiple myeloma (DREAMM-2): a two-arm, randomised, open-label, phase 2 study. Lancet Oncol. 2020;21(2):207-221. doi:10.1016/S1470-2045(19)30788-0

19. Eaton JS, Miller PE, Mannis MJ, Murphy CJ. Ocular adverse events associated with antibodydrug conjugates in human clinical trials. $J$ Ocul Pharmacol Ther. 2015;31(10):589-604. doi:10.1089/ jop.2015.0064

20. Lonial S, Lee HC, Badros AZ, et al. 1417 DREAMM-2: single-agent belantamab mafodotin (Belamaf) in patients with relapsed/refractory multiple myeloma (RRMM) - 1-year outcomes by prior therapies. Presented at: 62nd American Society of Hematology Annual Meeting and Exposition; December 5-8, 2020; virtual. Abstract 1417.

21. Popat R, Nooka A, Stockerl-Goldstein K, et al. DREAMM-6: safety, tolerability and clinical activity of belantamab mafodotin (Belamaf) in combination with bortezomib/dexamethasone (BorDex) in relapsed/refractory multiple myeloma (RRMM). Blood. 2020;136(suppl 1):19-20. doi:10.1182/ blood-2020-139332

22. Campbell KS, Cohen $A D$, Pazina $T$. Mechanisms of NK cell activation and clinical activity of the therapeutic SLAMF7 antibody, elotuzumab in multiple myeloma. Front Immunol. 2018;9:2551. doi:10.3389/ fimmu.2018.02551

23. Yu B, Jiang T, Liu D. BCMA-targeted immunotherapy for multiple myeloma. J Hematol Oncol. 2020;13(1):125. doi:10.1186/s13045-020-00962-7

Editor's Note: Interview quotes slightly modified for readability. 


\section{CONTINUING MEDICAL EDUCATION (CME)}

\section{Transplant-Associated Thrombotic Microangiopathy (TA-TMA): Integrating New Data to Improve Patient Outcomes}

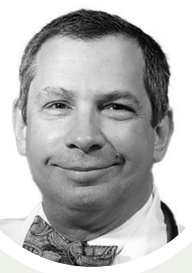

FACULTY

Sergio A. Giralt, MD, FACP

Professor of Medicine

Weill Cornell Medical College

Melvin Berlin Family Chair in Multiple

Myeloma

Deputy Head, Division of Hematologic

Malignancies

Chief Attending Physician, Adult Bone

Marrow Transplant Service

Memorial Sloan Kettering Cancer Center New York, NY

This activity was written by $\mathrm{PER}^{\circledR}$ editorial staff under faculty guidance and review.

The Q\&A portion of the activity was

transcribed from a recorded interview with the faculty and edited by faculty and PER ${ }^{\circledR}$ editorial staff for clarity.

\section{CMIE PROVIDER CONTACT}

INFORMATION

Physicians' Education Resource ${ }^{\circledR}$, LLC

2 Clarke Drive, Suite 110

Cranbury, NJ 08512

Toll-Free: 888-949-0045

Local: 609-378-3701

Fax: 609-257-0705

info@gotoper.com

\section{LEARNING OBJECTIVES}

Upon successful completion of this activity, you should be better prepared to:

- Review current diagnostic criteria for hematopoietic stem cell transplant-thrombotic microangiopathy (HSCT-TMA) and ways to differentiate it from other stem cell transplant-associated complications with overlapping symptoms

- Assess the role of dysregulated complement activation in the pathogenesis of HSCT-TMA

- Discuss the rationale concerning the application of complement-targeted therapies, including MASP2 inhibitors, in the treatment of HSCT-TMA

- Evaluate recent clinical trial data concerning the effectiveness of emerging therapies in the treatment of HSCT-TMA

RELEASE DATE: May 2021

EXPIRATION DATE: May 2022

\section{INSTRUCTIONS FOR PARTICIPATION /} HOW TO RECEIVE CREDIT

\section{Read this activity in its entirety.}

2. Go to https://www.gotoper.com/go/ta-tma-print21 to access and complete the posttest.

3. Answer the evaluation questions.

4. Request credit using the drop-down menu.

You may immediately download your certificate.

\section{FACULTY, STAFF, AND PLANNERS' DISCLOSURES}

In accordance with ACCME Guidelines, PER ${ }^{\circledR}$ has identified and resolved all COI for faculty, staff, and planners prior to the start of this activity by using a multistep process.

Disclosures (Dr Giralt): Grant Research Support: Actinium Pharmaceuticals, Amgen, Bristol Myers Squibb, Celgene Corporation, Janssen Pharmaceuticals, Pfizer, Sanofi; Other: Scientific Advisory Board: Actinium Pharmaceuticals, Amgen, Bristol Myers Squibb, Celgene Corporation, GlaxoSmithKline, Janssen Pharmaceuticals, Jazz Pharmaceuticals.

The staff of $P E R^{\circledR}$ have no relevant financial relationships with commercial interests to disclose.

OFF-LABEL DISCLOSURE AND DISCLAIMER

This activity may or may not discuss investigational, unapproved, or off-label use of drugs. Learners are advised to consult prescribing information for any products discussed. The information provided in this activity is for accredited continuing education purposes only and is not meant to substitute for the independent clinical judgment of a healthcare professional relative to diagnostic, treatment, or management options for a specific patient's medical condition. The opinions expressed in the content are solely those of the individual faculty members, and do not reflect those of $\mathrm{PER}^{\circledR}$ or any of the companies that provided commercial support for this activity.

This activity is funded by an educational grant from Omeros Corporation.

\section{ACCREDITATION/CREDIT DESIGNATION}

Physicians' Education Resource ${ }^{\circledR}$, LLC, is accredited by the Accreditation Council for Continuing Medical Education (ACCME) to provide continuing medical education for physicians. Physicians' Education Resource ${ }^{\circledR}$, LLC, designates this enduring material for a maximum of 0.25 AMA PRA Category 1 Credits $^{T M}$. Physicians should claim only the credit commensurate with the extent of their participation in the activity. 


\section{Transplant-Associated Thrombotic Microangiopathy (TA-TMA): Pathophysiology and Diagnostic Criteria}

An estimated 18,000 hematopoietic stem cell transplant (HSCT) procedures are performed in the United States each year. ${ }^{1,2}$ Transplant-associated thrombotic microangiopathy (TA-TMA) is the result of a systemic vascular endothelial injury following transplantation. The true incidence of TA-TMA is currently unknown due to a lack of awareness of the condition and due to non-standardized diagnostic criteria. Single center and registry studies have reported rates between 1 and $20 \%$. Severe TA-TMA, leading to multi-organ failure is associated with mortality rates above $80 \% .^{1,2}$ The clinical presentation of TA-TMA is similar to that of other TMAs and includes thrombocytopenia, Coombs-negative hemolysis, the presence of schistocytes on peripheral blood smear, and acute renal failure, mental status changes, or both. Early treatment can improve outcomes. However, clinical manifestations that overlap with those of other TMAs and the lack of specific diagnostic criteria hinder the early diagnosis of TA-TMA., ${ }^{1,2}$

The pathophysiology of TA-TMA is thought to involve an endothelial injury stemming from the conditioning regimen or other endothelial activating events (i.e, infections, drug or graft versus host disease). ${ }^{3}$ Endothelial activation induces cytokine release along with other proinflammatory changes and capillary flow obstruction, as a result of fibrin-related aggregates and platelet and leukocyte adhesion, which then lead to organ dysfunction. Other inflammatory changes, such as changes in nitric oxide (NO) levels and excessive complement activation, can also play a role in the pathophysiology of TA-TMA., ${ }^{4,5}$

The etiology of TA-TMA is multifactorial. The proposed risk factors for TA-TMA include (1) conditioning regimens with high-dose chemotherapy or total body irradiation (TBI); (2) immunosuppressive drugs, such as calcineurin inhibitors (CNIs) or mechanistic target of rapamycin (mTOR) inhibitors; (3) infections (eg, Epstein-Barr virus [EBV], cytomegalovirus [CMV], adenovirus, or Aspergillus); and (4) graftversus-host disease (GvHD).,2,6 Other patient characteristics are advanced age or sex, with females having a higher predisposition to TA-TMA. ${ }^{2}$ Clinical management of TA-TMA should include consideration of the individual characteristics and medical history of each patient, as well as the complex etiology of TA-TMA.

The aforementioned risk factors contributing to the development of TA-TMA are summarized in the "three-hit hypothesis." Patients with an inherited predisposition to complement activation or a pre-existing endothelial injury (hit 1) undergo a toxic conditioning regimen, resulting in endothelial injury (hit 2), and triggers activation of the complement system due to medication toxicity, alloreactivity, infections, antibodies, or some combination of all of these (hit 3). It is possible that the three-hit hypothesis will evolve over time, as more risk factors in the development of TA-TMA are identified.

Because TA-TMA has overlapping clinical features with other disorders, it is important to rule out (1) thrombotic thrombocytopenic purpura (TTP) by verifying normal levels of ADAMTS13 (>10\%) and normal von Willebrand factor (vWF) multimers; and (2) classic hemolytic uremic syndrome (HUS) caused by Shiga toxin-producing Escherichia coli (STEC), through stool culture and testing for Shiga toxin. ${ }^{7}$ Lastly, a modified version of the Ham test can differentiate transplant-associated atypical HUS (aHUS) from other TMAs, although this might not be needed in clinical practice.

Several diagnostic criteria have been developed to facilitate the early diagnosis of TA-TMA (Table). These criteria all require the identification of schistocytes in the peripheral blood smear in variable amounts, and additional criteria of microangiopathic hemolytic anemia. The overall TMA criteria proposed by Cho et al. ${ }^{8}$ adapted the components of the Blood and Marrow Transplant Clinical Trials Network and International Working Group of the European Society for Blood and Marrow Transplantation criteria (Table). More recently, Jodele et al. ${ }^{9}$ proposed a new set of diagnostic criteria, which include 3 additional criteria: renal and neurologic dysfunction, as measured by proteinuria levels ( $\geq 30 \mathrm{mg} / \mathrm{dL}$ as an early marker of kidney disease); hypertension (> 140/90 mmHg for adults and > 95th percentile for pediatric patients); and terminal complement activation, as confirmed by elevated levels of serum C5b-9.

Despite the proposed diagnostic criteria, diagnosis of TA-TMA remains very difficult, as it is common for patients to develop anemia and thrombocytopenia after receiving an HSCT. Schistocytes might be absent in these patients because of high vascular permeability and the extravasation of red blood cells into tissues. Furthermore, interpretation of the percentage of schistocytes can vary dramatically between institutions. ${ }^{12}$ Different institutions have also adapted different diagnostic criteria, suggesting the need for a unified approach to the diagnosis of TA-TMA.

\section{MolecularTargets and Novel Treatment Options in TA-TMA}

Therapeutic plasma exchange (TPE) is frequently used for the treatment of TATMA. Although it can provide temporary benefit to patients with TA-TMA and an acute response, studies have shown varying response rates with TPE. ${ }^{1}$ TPE may improve hematologic criteria such as platelet count, hemoglobin and haptoglobin levels, and lactate dehydrogenase (LDH) levels, and may reduce inflammatory cytokine levels and thrombotic markers, but it does not ameliorate the ongoing tissue and organ damage or affect survival. ${ }^{1,13-16}$ Therefore, there is an urgent need for alternative treatment options for TA-TMA.

Endothelial injury resulting from an HSCT activates the leptin pathway (LP) of complement. Mannose-binding lectinassociated serine protease (MASP2), the effector enzyme of the LP, was recently found to be involved in the development of TTP, aHUS, and TA-TMA. ${ }^{17}$ Plasma MASP2 levels were significantly increased in all patients with TMA compared with 


\section{TABLE. Diagnostic Criteria forTA-TMA ${ }^{\mathbf{8}-11}$}

\begin{tabular}{|c|c|c|c|c|}
\hline Clinical marker & $\begin{array}{l}\text { International } \\
\text { Working Group }\end{array}$ & $\begin{array}{l}\text { Blood and Marrow Transplant } \\
\text { Clinical Trials Network }{ }^{11}\end{array}$ & $\begin{array}{l}\text { Overall TMA grouping } \\
\left(0-\text { TMA }^{8}\right.\end{array}$ & $\begin{array}{l}\text { Criteria proposed } \\
\text { by Jodele et al. }{ }^{9}\end{array}$ \\
\hline Schistocytes & $>4 \%$ & $>2$ per hpf & $>2$ per hpf & Present \\
\hline Platelet count & $\begin{array}{l}<50,000 / \mathrm{mm}^{3} \text { or } \\
<50 \% \text { of normal } \\
\text { baseline }\end{array}$ & NS & $\begin{array}{l}<50,000 / \mathrm{mm}^{3} \text { or }<50 \% \text { of } \\
\text { normal baseline }\end{array}$ & $\begin{array}{l}<50,000 / \mathrm{mm}^{3} \text { or } \\
<50 \% \text { of normal } \\
\text { baseline }\end{array}$ \\
\hline LDH & Increased & Increased & Increased & Increased \\
\hline Haptoglobin & Decreased & NS & Decreased & $\begin{array}{l}\text { Low hemoglobin/ } \\
\text { anemia }\end{array}$ \\
\hline Transfusions & Increased & NS & NS & Increased \\
\hline Creatinine & NS & $2 \times$ baseline & NS & NS \\
\hline Coombs test & NS & Negative & Negative & NS \\
\hline Coagulation studies & Normal & Normal & Normal & NS \\
\hline ADAMTS13 mutation & NS & NS & NS & $<5 \%$ to $10 \%$ \\
\hline
\end{tabular}

hpf, high-power field; LDH, lactate dehydrogenase; NS, not significant.

controls, although they were relatively lower in patients with TA-TMA than in patients with other TMAs. There was also a positive correlation between serum C5b-9 and MASP2 levels in patients with TTP $\left(\mathrm{R}^{2}=0.419\right)$, but not in patients with the non-TTP types of TMA, including TATMA $\left(\mathrm{R}^{2}=0.035 ; \mathrm{n}=9\right)$.

Narsoplimab, a specific inhibitor of MASP2 and the LP, was evaluated in an open-label, phase 2, single-arm study for the treatment of patients with TA-TMA, and the results were presented at the 2021 Transplantation and Cellular Therapy Meeting. ${ }^{18}$ The data comprised the full analysis set of patients receiving at least 1 dose of narsoplimab $(n=28)$ and those in the per-protocol population, who received the protocol-specified narsoplimab treatment for at least 4 weeks $(n=23)$.

Results showed that treatment with narsoplimab resulted in an objective response rate (ORR) of $61 \%(95 \% \mathrm{CI}$, $40.6 \%-78.5 \% ; P<.0001)$ in the full analysis set of patients $(\mathrm{n}=28)$ and an ORR of $74 \%$ (95\% CI, $51.6 \%-89.8 \%$; $>$ <.0001) in the per-protocol group $(n=23)$. The post HSCT-TMA 100-day survival rates were $68 \%$ and $83 \%$, respectively; in those who had complete responses (CRs), the 100day survival rate was $94 \%$. The median overall survival (OS) in the full analysis, perprotocol, and CR populations were 274 days, 361 days, and not estimable, respectively. ${ }^{18}$ Patients also showed improvement in TMA markers, such as platelet counts, LDH, and haptoglobin levels. Narsoplimab had an acceptable safety profile, and the most common adverse events (AEs) were nausea $(25.0 \%)$, vomiting $(32.1 \%)$, diarrhea $(32.1 \%)$, neutropenia $(25.0 \%)$, and pyrexia $(35.7 \%)$. Six deaths were reported in the study, with causes commonly related to HSCT, including neutropenic sepsis, septic shock, acute myeloid leukemia, TMA, and GvHD.

An updated analysis of the study was presented at the 2021 Annual Meeting of the European Society for Blood and Marrow Transplantation (EBMT). ${ }^{19}$ Response to narsoplimab was found to be consistent across different subgroups regardless of age, sex, and coexisting conditions such as GvHD, significant infection, multiple-organ TMA involvement, mismatched donor, and transfusion within 2 weeks before the first narsoplimab dose. Narsoplimab was granted priority designation by the FDA for the treatment of TA-TMA in January 2021.20

Eculizumab (ECU) is a terminal complement inhibitor that was approved by the
FDA for the treatment of aHUS in $2011 .{ }^{21} \mathrm{It}$ is a humanized monoclonal antibody against complement $\mathrm{C} 5$, blocking the cleavage of $\mathrm{C} 5$ to $\mathrm{C} 5 \mathrm{a}$ and $\mathrm{C} 5 \mathrm{~b}$, and therefore preventing complement-mediated endothelial damage. ${ }^{22}$ In a retrospective study of 10 patients with TA-TMA, early treatment with ECU after allogeneic HSCT neither altered the disease process nor restored organ function, but it did prolong survival. The overall hematologic response rate was $70 \%$ and the OS rate was $60 \%$. Only 1 patient achieved a CR with corresponding organ recovery. A systematic review and meta-analysis that pooled data from 6 studies $(n=116)$ revealed that ECU improves survival rate and overall response rate in patients with TA-TMA and exhibits a manageable safety profile. ${ }^{23}$ The estimated overall response rate, $\mathrm{CR}$ rate, and survival rate at the last follow-up (SR) were $71 \%, 32 \%$, and $52 \%$, respectively. Infection was the most common AE, and the main cause of death, together with GvHD. Randomized, controlled clinical trials and more extensive prospective cohort studies are needed for better assessment of the efficacy and safety of ECU in TA-TMA. These findings also suggest that monitoring serum C5b-9 levels is crucial in TA-TMA and may help to identify patients who could benefit from ECU therapy. 


\section{Clinical Considerations for the Diagnosis and Management of TA-TMA in Pediatric Patients}

In pediatric patients, TA-TMA is usually observed soon after allogeneic HSCT, with a median diagnosis at 35 to 47 days posttransplant. Between $88 \%$ and $92 \%$ of cases occur within the first 100 days following transplant..$^{7,24,25,26}$ Patients who receive autologous HSCT may develop TA-TMA even earlier, with a median diagnosis at 18 days posttransplant.?

Hypertension is usually the first clinical sign of TA-TMA. However, hypertension is also fairly common after HSCT, particularly in recipients of CNIs. In the pediatric population, TA-TMA should be suspected when a patient requires additional antihypertensive medication. ${ }^{7}$ Other clinical features include thrombocytopenia (particularly refractoriness to platelet transfusion), elevated LDH, proteinuria, anemia, and decreasing haptoglobin appearing shortly after. During the initial 100 days following HSCT, routine monitoring of these markers is recommended. Dvorak et al. ${ }^{7}$ have proposed the "TA-TMA triad"; that is, hypertension, thrombocytopenia, and elevated LDH, which should trigger a more detailed evaluation for TA-TMA, including complement studies and organ function assessment. ${ }^{7,24}$

The recommendations associated with the TA-TMA triad are based on prospective studies that have monitored patients postHSCT to determine risk factors associated with moderate and severe TMA, as well as disease markers for early diagnosis. ${ }^{24,26}$ In one study, severe TMA occurred in $18 \%$ of HSCT recipients. Hypertension, elevated LDH levels, and proteinuria were determined to be the earliest markers of severe TMA. ${ }^{24}$ Proteinuria (>30 mg/ $\mathrm{dL}$ ) and marked terminal complement activation at the time of TMA diagnosis, as determined by elevated serum C 5 b- 9 levels, were associated with less than $20 \%$ survival at year 1 posttransplant and $9 \%$ OS. Patients without proteinuria and with a normal serum concentration of C5b-9 have a longer OS $(P<.01)$.
In a multicenter, large cohort study of pediatric patients $(\mathrm{N}=614)$ who received prospective TA-TMA screening at 13 centers, 98 patients $(16 \%)$ were diagnosed with TA-TMA at a median of 22 days following transplant. ${ }^{27}$ TA-TMA diagnosis was based on the presence of more than 4 of 7 laboratory/clinical markers of TATMA concurrently or tissue histology showing TA-TMA. Overall, patients with TA-TMA had significantly lower OS and increased transplant-related complications that required intensive care support, including respiratory failure and acute kidney injury.

In this study, the association of neuroblastoma with TA-TMA was also investigated. ${ }^{27}$ A total of 72 patients had undergone autologous HSCT for neuroblastoma. None of the 21 patients who received a single transplant was diagnosed with TA-TMA. Among the patients who were scheduled to receive tandem HSCT, 13 (25\%) developed TA-TMA. As patients with neuroblastoma receiving tandem HSCT appear to be at high risk for developing TA-TMA, further investigations of the associated mechanisms of endothelial injury might facilitate the improvement of patient outcomes.

In a recent study, the treatment of pediatric patients $(\mathrm{N}=64)$ with high-risk TA-TMA and multiorgan injury with the complement blocker ECU led to a significant improvement in 1-year post-HSCT survival compared with a previously reported untreated cohort with the same high-risk features (66\% vs $16.7 \%$, respectively). ${ }^{28}$ A median of 11 doses of ECU treatment was required for patients to respond to and benefit from the therapy, and TA-TMA was resolved at a median of 66 days, resulting in treatment discontinuation. Patients with higher serum C5b-9 levels at the start of treatment were less likely to respond to ECU (odds ratio, $0.15 ; P=.0014)$, and required more doses $(\mathrm{r}=0.43 ; P=.0004)$. Patients with gastrointestinal (GI) bleeding required the highest number of ECU doses compared with those without GI bleeding (20 vs 9; $P=.0015$ ). They also had the lowest response rates to ECU and lowest 1-year survival rates compared with patients without GI bleeding (35\% vs $80 \%, P=.0004$, and $44 \%$ vs $78 \%$; $P=.01$, respectively). More than $70 \%$ of the survivors experienced proteinuria on long-term follow-up. Although complement blockade with ECU was an effective therapeutic strategy for a high proportion of patients with high-risk TA-TMA, some patients with severe disease did not achieve a CR, suggesting the need for additional actionable endothelial injury pathways.

Despite limitations, these studies provide crucial data and emphasize the importance of routine, scheduled monitoring of pediatric patients undergoing HSCT for TA-TMA. Complement activation, as measured by elevated blood serum levels of C5b-9 and proteinuria, increases the risk of developing TA-TMA. Patient stratification would allow early interventions and prolong survival in both pediatric and adult patients. Prospective TA-TMA screening is also critical to enforce standardized diagnostic and care guidelines for patients who are at risk of developing TA-TMA.

\section{KEY REFERENCES}

1. Chapin J, Shore T, Forsberg P, Desman G, Van Besien $\mathrm{K}$, Laurence J. Hematopoietic transplant-associated thrombotic microangiopathy: case report and review of diagnosis and treatments. Clin Adv Hematol Oncol. 2014;12(9):565-573.

7. Dvorak CC, Higham C, Shimano KA. Transplantassociated thrombotic microangiopathy in pediatric hematopoietic cell transplant recipients: a practical approach to diagnosis and management. Front Pediatr. 2019;7:133. doi:10.3389/fped.2019.00133

19. Rambaldi A, Claes K, Goh Y. Narsoplimab (OMS721), a MASP-2 inhibitor, for the treatment of adult hematopoietic stem cell transplant-associated thrombotic microangiopathy (HSCT-TMA): subgroup analyses. Paper presented at: 47th Annual Meeting of the European Society for Blood and Marrow Transplantation; March 14-17, 2021; virtual.

27. Dandoy CE, Rotz $S$, Alonso $P B$, et al. A pragmatic multi-institutional approach to understanding transplantassociated thrombotic microangiopathy after stem cell transplant. Blood Adv. 2021;5(1):1-11. doi:10.1182/ bloodadvances.2020003455

28. Schoettler M, Lehmann L, Li A, Ma C, Duncan C. Thrombotic microangiopathy following pediatric autologous hematopoietic cell transplantation: a report of significant end-organ dysfunction in eculizumab-treated survivors. Biol Blood Marrow Transplant 2019;25(5):e163-e168. doi:10.1016/j.bbmt.2018.12.840

I

For full reference list, visit https://www.gotoper.com/go/ta-tmaprint21 
Sanc 'ieu

Stay informed of the latest

data \& practice advice.

Watch: cancernetwork.com/oncview

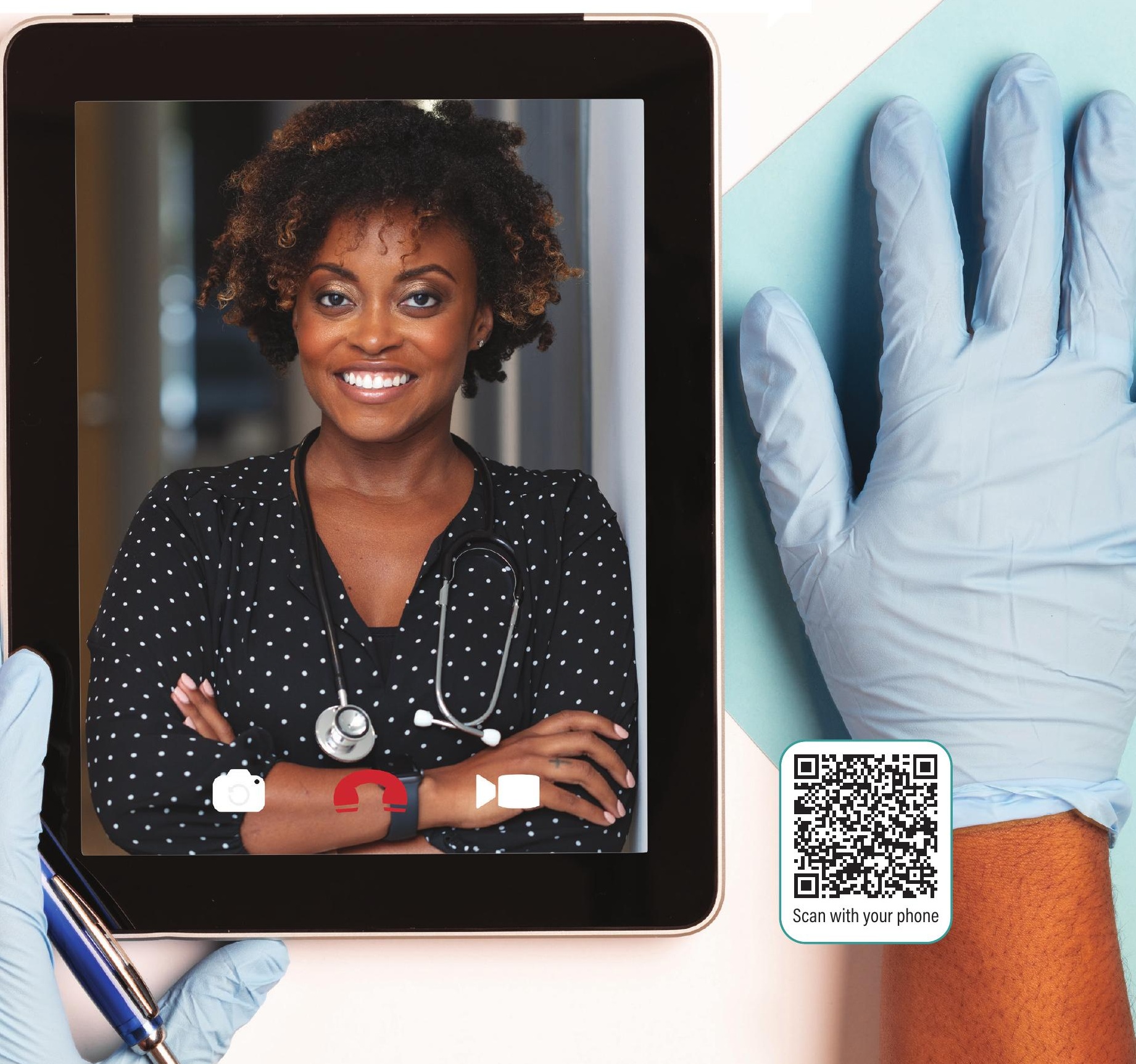




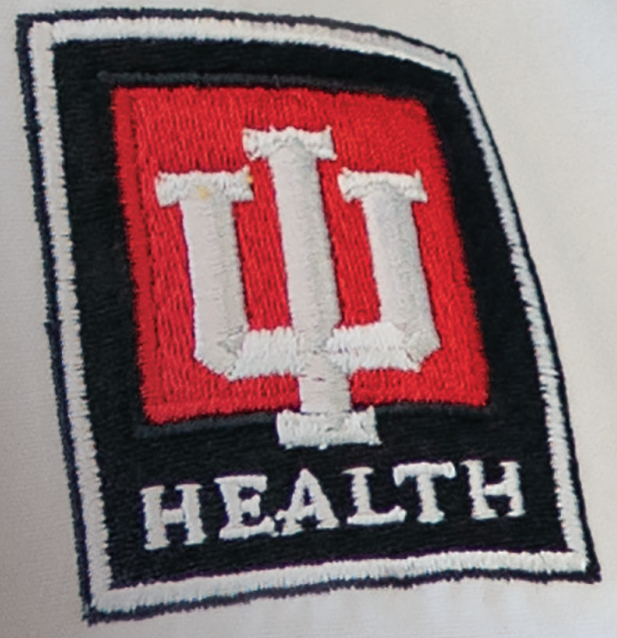

Partner with a nationally recognized leader in blood and bone marrow stem cell transplants.

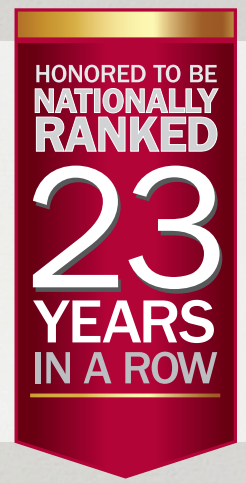


